\title{
Modelling effect of toxicant in a three-species food-chain system incorporating delay in toxicant uptake process by prey
}

\author{
O. P. Misra ${ }^{1}$ A. Raveendra Babu ${ }^{1}$
}

Received: 30 March 2016/Accepted: 4 April 2016/Published online: 23 April 2016

(C) Springer International Publishing Switzerland 2016

\begin{abstract}
In this paper, a mathematical model is proposed and analyzed to study the effect of toxicant in a threespecies food-chain system incorporating delay in toxicant uptake process by prey population. The model is formulated by using the system of non linear ordinary differential equations. In the model, it is assumed that the growth rate of prey population is affected by organismal toxicant. In this paper, we have introduced distributed delay in the environmental toxicant in the model. The distributed delay differential equations, though simple in structure, possess a rich array of solutions. The models are being analyzed by using variational matrix and Liapunov functions. The conditions for local and global stability of the equilibrium points are obtained. A region of attraction is being found for global asymptotic stability of the equilibrium points. Also, a Hopf bifurcation analysis has been performed with respect to key parameters for non-trivial equilibrium points. Furthermore, we support our analytical findings with numerical simulations.
\end{abstract}

Keywords Food-chain - Toxicant - Distributed delay · Equilibrium $\cdot$ Stability $\cdot$ Hopf-bifurcation

Mathematics Subject Classification 93A30 - 92D40 . 34D20

A. Raveendra Babu

raveendra96@hotmail.com

O. P. Misra

misra_op@rediffmail.com

1 Department, School of Mathematics and Allied Sciences, Jiwaji University, Gwalior 474 011, India

\section{Introduction}

It is now well known that the environmental pollution is caused by several different types of industrial discharges and chemical spills which pollute the air and contaminate the aquatic habitats (streams, rivers, lakes and oceans) with varieties of chemicals and toxicants such as arsenic, cadmium, lead, zinc, copper, iron, mercury, etc. These toxicants are responsible for the decrease in the growth rate of the exposed species and therefore, it is causing damage to terrestrial and aquatic populations (Dubey and Hussain 2006). Therefore, it is important to study the effects of toxicants on populations inorder to find a theoretical threshold value of the organismal concentration of toxicant, which will predict the existence or extinction of a population or community in future.

Uncontrolled emission of toxicant into the environment is responsible for the extinction of many species and if it is not controlled then the species which are at the verge of extinction may vanish completely. Beyond effects on direct grazers, toxins may accumulate in and be transferred through marine food chains, affecting consumers at higher trophic levels, including fish, seabirds, and marine mammals (Turner and Tester 1997). According to Hladun et al. (2012) study that the Selenium (Se) is a metalloid that occurs naturally in certain alkaline soils from shale deposits of prehistoric inland seas. Agricultural water drainage dissolves Se from these naturally seleniferous soils and has caused the buildup of selenate, the predominant and bioavailable form of Se. One of the worst cases of Se pollution occurred at the Kesterson Reservoir in the San Joaquin Valley, a major drainage site for many agricultural regions of California. There is good evidence that Se accumulation can have negative effects on plant growth, insect herbivores, their predators and parasites, 
and the detritivores that feed on decaying plant and animal tissues.

In an experimental study of marine food chains (Zhang et al. 2013) the varying concentration of $\mathrm{Hg}$ in the microalgae species as well as the selectivity of zooplankton to the algal diet resulted in varying $\mathrm{Hg}$ accumulation in three zooplankton species i.e., feeding selectivity plays a key role in the transfer of $\mathrm{MeHg}$ along marine food chains. The destruction of zooplankton can indirectly affect fish diversity of reservoirs (Perez-Legaspi and Rico-Martnez 2001). The dynamic behaviour of the stressed ecosystem can be much more complicated than that of the unstressed system. In last few decades, many efforts have been made to study the effects of toxicant on a three-species food chain systems using mathematical models (Thomann et al. 1984; Thomann and Connolly 1984; Hallam and De Luna 1984). All these studies are related to the instantaneous effects of the toxicant on the species and/or on their biomass. The uptake of environmental toxicant by the resource-biomass may not be instantaneous. Therefore, the consideration of delay effect in such situation is very logical and close to real systems.

Often the dynamical behavior of biological systems depend on their past history and therefore it is important to incorporate continuous or distributed time delays in the model to study most of the biological processes. In general, delay differential equations exhibit more complicated dynamics on stability, periodic structure, bifurcation, and so on. The effect of the distributed delay on the stability of the model has been investigated by Shi et al. (2013). In last few decades, several investigators have proposed and analyzed mathematical models considering distributed delays in the growth dynamics of biological species (Arino et al. 2007; He and Ruan 1998; Bernard et al. 2001; Rasmusse et al. 2003; Ma and Takeuchi 1998; Gakkhar and Sahani 2009; Khare et al. 2011). Almost no studies have been conducted to investigate the modelling effect of toxicant in a three-species food-chain system incorporating delay in toxicant uptake process by prey.

Therefore in this paper we have studied the dynamical behaviour of three species food chain system under pollution stress considering the delay in toxicant uptake process by exposed prey population using mathematical model.

\section{Mathematical model}

We have incorporated delay in toxicant uptake process by exposed prey population in a three-species food-chain system. In the model, it is assumed that the growth rate of prey population is negatively affected by a organismal toxicant. In the model, we have introduced distributed delay in the environmental toxicant. The underlying system consists of the state variables are $x(t)$, the density of the prey population; $y(t)$, the density of the intermediate predator population; $z(t)$, the density of the top predator population; $C_{E}(t)$, the environmental toxicant concentration; $C_{0}(t)$, the organism toxicant concentration; and $R_{1}(t)$, the distributed delay.

Taking these as state variables, we formulate the mathematical model using following system of non linear ordinary differential equations in order to study the role of distributed delay in toxicant uptake process by exposed prey population in a three-species food-chain system:

Main Model 1:

$$
\begin{aligned}
& \frac{d x}{d t}=x\left(s_{0}-s_{1} C_{0}-\frac{x}{k}\right)-\frac{q_{1} x y}{1+x} \\
& \frac{d y}{d t}=\frac{q_{2} x y}{1+x}-\frac{q_{3} y z}{1+y}-r_{0} y-r_{1} y^{2} \\
& \frac{d z}{d t}=\frac{q_{4} y z}{1+y}-d_{1} z-d_{2} z^{2} \\
& \frac{d C_{E}}{d t}=q_{0}-a_{1} C_{E}-a_{2} x R_{1}+v x C_{0} \\
& \frac{d C_{0}}{d t}=a_{2} x R_{1}-b_{1} C_{0}-v x C_{0} \\
& \frac{d R_{1}}{d t}=\gamma_{1}\left(C_{E}-R_{1}\right)
\end{aligned}
$$

The initial conditions are

$x(0)>0, y(0)>0, z(0)>0, C_{E}(0)=f(0) \geq 0, C_{0}(0) \geq 0, R_{1}(0)>0$.

In the model, the growth equation for the prey population is logistic growth and term $s_{0}$ is the intrinsic growth rate of prey and $k$ is the carrying capacity of environment for the prey population. $r_{0}$ and $d_{1}$ are the natural death rates of intermediate and top predator populations respectively. $r_{1}$ and $d_{2}$ are the intraspecies competition coefficients among intermediate and top predator populations respectively.

In the model $q_{i} u /(1+u),(i=1,2,3,4 ; u=x$ and $y)$, account for the interactions between two different species, representing the Holling type-II functional response. This functional response is parameterized by the constant $q_{i} . q_{1}$ and $q_{3}$ are the predation rate of prey and intermediate predator populations respectively. $q_{2}$ and $q_{4}$ are the intermediate and top predators growth rates as result of feeding on prey and intermediate predator populations respectively. $s_{1}$ is the decreasing rate of the intrinsic growth rate associated with the uptake concentration of organismal toxicant. $q_{0}$ is the external input of toxicant into environment. The toxicant are assumed to wash out from the environment as well as from the organism at rate $a_{1}$ and $b_{1}$, respectively. $a_{2}$ is the removal rate of toxicant from environment due to uptake by the prey species. $v$ is the removal rate of toxicant from the prey species due to its death. It is assumed that the growth rate of uptake concentration of 
organism $C_{0}(t)$ increases by the same amount as the depletion rate of toxicant concentration $C_{E}(t)$ in the environment and is assumed to be proportional to the prey population as well as the environmental concentration of the toxicant (i.e., $a_{2} x C_{E}$ ). Also it is assumed that the uptake concentration of organism may decrease with rate coefficient $v \geq 0$ due to decaying of prey population and which (i.e., $v x C_{0}$ ) may reenter into the environment (Shukla and Dubey 1997).

In the model, we have introduced distributed delay $R_{1}(t)$ in the term ' $a_{2} x C_{E}$ ' which describes the uptake of environmental toxicant by the prey population (Khare et al. 2009, 2011).

$R_{1}(t)=\int_{-\infty}^{t} \gamma_{1} \exp \left(-\gamma_{1}(t-s)\right) C_{E}(s) d s$

where $s \in(-\infty, 0], \gamma_{1}$ is delay parameter. Now, if the effect of distributed delay is not considered in the above Model 1, then we have the following Model 2 for the effect of toxicant on three species food chain system:

Model 2: (With Toxic Effect and No Delay)

$\frac{d x}{d t}=x\left(s_{0}-s_{1} C_{0}-\frac{x}{k}\right)-\frac{q_{1} x y}{1+x}$

$\frac{d y}{d t}=\frac{q_{2} x y}{1+x}-\frac{q_{3} y z}{1+y}-r_{0} y-r_{1} y^{2}$

$\frac{d z}{d t}=\frac{q_{4} y z}{1+y}-d_{1} z-d_{2} z^{2}$

$\frac{d C_{E}}{d t}=q_{0}-a_{1} C_{E}-a_{2} x C_{E}+v x C_{0}$

$\frac{d C_{0}}{d t}=a_{2} x C_{E}-b_{1} C_{0}-v x C_{0}$

with the initial conditions as $x(0)>0, y(0)>0, z(0)>0, C_{E}(0) \geq 0, C_{0}(0) \geq 0$, where, the state variables and parameters are the same as defined for the Model 1. Now, if the effect of toxicant is not considered in the above Model 2, then we have the following Model 3 for three species food chain system:

Model 3: (Without Toxic Effect and No Delay)

$\frac{d x}{d t}=x\left(s_{0}-\frac{x}{k}\right)-\frac{q_{1} x y}{1+x}$

$\frac{d y}{d t}=\frac{q_{2} x y}{1+x}-\frac{q_{3} y z}{1+y}-r_{0} y-r_{1} y^{2}$

$\frac{d z}{d t}=\frac{q_{4} y z}{1+y}-d_{1} z-d_{2} z^{2}$

with the initial conditions as $x(0)>0, y(0)>0, z(0)>0$, where, the state variables and parameters are the same as defined for the Model 1.

Lemma 1 (Wang and Pang 2008) Suppose $n$ is a positive integer and $f_{i}\left(t, x_{1}, x_{2}, \ldots, x_{n}\right), \quad(i=1,2,3, \ldots, n)$ are smooth functions. If $\left.\quad f_{i}\right|_{x_{i}=0, \Omega \in R_{+0}^{n}} \geq 0 \quad$ (where
$\left.\Omega=\left(x_{1}, x_{2}, \ldots, x_{n}\right)^{T} \in R^{n}\right)$, then $R_{+0}^{n}$ is an invariant domain of the following equations:

$\frac{d x_{i}}{d t}=f_{i}\left(t, x_{1}, x_{2}, \ldots, x_{n}\right),(i=1,2,3, \ldots, n)$

If $\left.f_{i}\right|_{x_{i}=0, \Omega \in R_{-0}^{n}} \leq 0$ (where $\Omega=\left(x_{1}, x_{2}, \ldots, x_{n}\right)^{T} \in R^{n}$ ), then $R_{-0}^{n}$ is an invariant domain of the above equations.

\section{Theorem 1}

$R_{+0}^{6}:=\left\{\left(x, y, z, C_{E}, C_{0}, R_{1}\right)^{T} \mid x \geq 0, y \geq 0, z \geq 0, C_{E} \geq 0, C_{0} \geq 0, R_{1} \geq 0\right\}$

is an invariant domain of Eqs. (1)-(6).

Proof Denote that $\Omega:=\left(x, y, z, C_{E}, C_{0}, R_{1}\right)^{T}$. For the Model 1, we notice that

$\left.f_{1}\right|_{x=0, \Omega \in R_{+0}^{6}}=\left.\left(x\left(s_{0}-s_{1} C_{0}-\frac{x}{k}\right)-\frac{q_{1} x y}{1+x}\right)\right|_{x=0, \Omega \in R_{+0}^{6}}=0 \geq 0$,

$\left.f_{2}\right|_{y=0, \Omega \in R_{+0}^{6}}=\left.\left(\frac{q_{2} x y}{1+x}-\frac{q_{3} y z}{1+y}-r_{0} y-r_{1} y^{2}\right)\right|_{y=0, \Omega \in R_{+0}^{6}}=0 \geq 0$,

$\left.f_{3}\right|_{z=0, \Omega \in R_{+0}^{6}}=\left.\left(\frac{q_{4} y z}{1+y}-d_{1} z-d_{2} z^{2}\right)\right|_{z=0, \Omega \in R_{+0}^{6}}=0 \geq 0$.

$\left.f_{4}\right|_{C_{E}=0, \Omega \in R_{+0}^{6}}=\left.\left(q_{0}-a_{1} C_{E}-a_{2} x R_{1}+v x C_{0}\right)\right|_{C_{E}=0, \Omega \in R_{+0}^{6}}$ $=q_{0}+v x C_{0}>0$,

$\left.f_{5}\right|_{C_{0}=0, \Omega \in R_{+0}^{6}}=\left.\left(a_{2} x R_{1}-b_{1} C_{0}-v x C_{0}\right)\right|_{C_{0}=0, \Omega \in R_{+0}^{6}}=a_{2} x R_{1}>0$,

$\left.f_{6}\right|_{R_{1}=0, \Omega \in R_{+0}^{6}}=\left.\left(\gamma_{1}\left(C_{E}-R_{1}\right)\right)\right|_{R_{1}=0, \Omega \in R_{+0}^{6}}=0 \geq 0$.

By the Lemma 1 , we get that $R_{+}^{6} 0$ is an invariant domain of Eqs. (1)-(6).

\section{Boundedness of the models}

To analyze the models 1, 2 and 3 , in this section we need the bounds of dependent variables involved. First we see the boundedness of Model 3, Model 2 and finally Model 1. So here, wefind the region of attraction for all the models in the following lemma.

Lemma 2 The set

$$
\begin{gathered}
\Omega=\left\{\left(x, y, z, C_{E}, C_{0}, R_{1}\right) \in R_{+}^{6}: x(t) \leq s_{0} k, x(t)+\frac{q_{1}}{q_{2}} y(t)+\frac{q_{1} q_{3}}{q_{2} q_{4}} z(t) \leq K_{1},\right. \\
\left.C_{E}(t)+C_{0}(t) \leq K_{2}, C_{E}(t)+R_{1}(t) \leq K_{5}, C_{E}(t) \geq K_{3}, x(t)+C_{E}(t) \geq K_{4}\right\}
\end{gathered}
$$

is a region of attraction for all solutions initiating in the interior of the positive region, where $K_{1}=s_{0} k\left(s_{0}+1\right) / \Phi_{11}$ and $\Phi_{11}=\min \left\{1, r_{0}, d_{1}\right\}, K_{2}=\left(q_{0}+1\right) / \Phi_{12}$ and $\phi_{12}=$ $\min \left\{a_{1}, b_{1}\right\}, K_{3}=q_{0} / \phi_{13}$ and $\phi_{13}=\left(a_{1}+a_{2} s_{0} k\right), K_{4}=$ $\left(q_{0}-q_{1} K_{1}\right) / \phi_{14}$ and $\phi_{14}=\max \left\{x_{1} K_{2}, a_{1}+a_{2} s_{0} k\right\}, K_{5}=$ $\left(q_{0}+v K_{1} K_{2}\right) / \phi_{15}$ and $\phi_{15}=\min \left\{a_{1}-\gamma_{1}, \gamma_{1}\right\}$ and $a_{1}>\gamma_{1}$. 
Proof From Eq. (12) we get, $d x / d t \leq x\left(s_{0}-\frac{x}{k}\right)$ then by the usual comparison theorem, we get as $t \rightarrow \infty$,

$x \leq s_{0} k$

Now, let us consider the following function:

$W_{11}(t)=x(t)+\frac{q_{1}}{q_{2}} y(t)+\frac{q_{1} q_{3}}{q_{2} q_{4}} z(t)$

by using Eqs. (12)-(14), we get $d W_{11} / d t+\Phi_{11} W_{11}$ $\leq s_{0} k\left(s_{0}+1\right)$ where $\Phi_{11}=\min \left\{1, r_{0}, d_{1}\right\}$ and then by the usual comparison theorem, we get as $t \rightarrow \infty, W_{11}(t)$ $\leq s_{0} k\left(s_{0}+1\right)$ and hence,

$x(t)+\frac{q_{1}}{q_{2}} y(t)+\frac{q_{1} q_{3}}{q_{2} q_{4}} z(t) \leq K_{1}$

where $K_{1}=s_{0} k\left(s_{0}+1\right) / \Phi_{11}$.

Now, let us consider the function: $W_{12}(t)=C_{E}(t)+$ $C_{0}(t)$ by using Eqs. (10)-(11), we get $d W_{12} / d t+\Phi_{12} W_{12} \leq$ $\left(q_{0}+1\right)$ where $\Phi_{12}=\min \left\{a_{1}, b_{1}\right\}$ and then by the usual comparison theorem, we get as $t \rightarrow \infty, W_{12}(t) \leq\left(q_{0}+\right.$ $1) / \phi_{12}$ and hence,

$C_{E}(t)+C_{0}(t) \leq K_{2}$

where $K_{2}=\left(q_{0}+1\right) / \phi_{12}$.

Now, let us consider the function: $W_{15}(t)=C_{E}(t)+$ $R_{1}(t)$ by using Eqs. (4) and (6), we get

$\frac{d W_{15}}{d t}+\phi_{15} W_{15} \leq q_{0}+v K_{1} K_{2}$

where $\phi_{15}=\min \left\{a_{1}-\gamma_{1}, \gamma_{1}\right\}$ and $a_{1}>\gamma_{1}$ then by the usual comparison theorem, we get as $t \rightarrow \infty, W_{15}(t) \leq$ $\left(q_{0}+v K_{1} K_{2}\right) / \phi_{15}$ and hence,

$C_{E}(t)+R_{1}(t) \leq K_{5}$

where, $K_{5}=\left(q_{0}+v K_{1} K_{2}\right) / \phi_{15}$.

From Eq. (10) we get, $d C_{E} / d t+\phi_{13} C_{E} \geq q_{0}$ where $\phi_{13}=\left(a_{1}+a_{2} s_{0} k\right)$ then by the usual comparison theorem, we get as $t \rightarrow \infty$,

$C_{E}(t) \geq K_{3}$

where $K_{3}=q_{0} / \phi_{13}$. Now, let us consider the function: $W_{14}(t)=x(t)+C_{E}(t)$ by using Eqs. (7) and (10), we get $d W_{14} / d t+\Phi_{14} W_{14} \geq\left(q_{0}-q_{1} K_{1}\right)$ where $\Phi_{14}=\max \left\{s_{1} K_{2}\right.$ $\left.+s_{0}, a_{1}+a_{2} s_{0} k\right\}$ and then by the usual comparison theorem, we get as $t \rightarrow \infty, W_{14}(t) \geq\left(q_{0}-q_{1} K_{1}\right) / \phi_{14}$ and hence,

$x(t)+C_{E}(t) \geq K_{4}$

where $K_{4}=\left(q_{0}-q_{1} K_{1}\right) / \phi_{14}$.

Hence the solution of the models 1, 2 and 3 are bounded in $\Omega$.

In the next section we study the dynamical behavior of three models.

\section{Analysis of Model 1}

\section{Equilibria of Model 1}

The Model 1 has four non negative equilibria in $x, y, z, C_{E}$, $C_{0}, R_{1}$ space namely, $\check{E_{10}}\left(0,0,0, \check{C_{E}}, 0, \check{R_{1}}\right), \hat{E_{11}}\left(\hat{x}, 0,0, \hat{C_{E}}\right.$, $\left.\hat{C_{0}}, \hat{R_{1}}\right), \quad \overline{E_{12}}\left(\bar{x}, \bar{y}, 0, \overline{C_{E}}, \overline{C_{0}}, \overline{R_{1}}\right) \quad$ and $\quad E_{13}^{*}\left(x^{*}, y^{*}, z^{*}, C_{E}^{*}\right.$, $\left.C_{0}^{*}, R_{1}^{*}\right)$. The existence of $E_{10}$ is obvious. We prove the existence of $\hat{E_{11}}, \overline{E_{12}}$ and $E_{13}^{*}$ as follows:

- Existence of $\check{E_{10}}=\left(0,0,0, \check{C_{E}}, 0, \check{R_{1}}\right)$ from (4),

$\check{C_{E}}=\frac{q_{0}}{a_{1}}$

from (6),

$\check{C_{E}}=\check{R_{1}}$.

- Existence of $\hat{E_{11}}=\left(\hat{x}, 0,0, \hat{C_{E}}, \hat{C_{0}}, \hat{R_{1}}\right)$ from (1),

$\hat{C_{0}}=\frac{1}{s_{1}}\left(s_{0}-\frac{\hat{x}}{k}\right)$

$\hat{C_{0}}>0$ if $s_{0} k>\hat{x}$. Addition of (4) and (5), and using (17), we get

$\hat{C_{E}}=\frac{1}{a_{1}}\left(q_{0}-\frac{b_{1}}{s_{1}}\left(s_{0}-\frac{\hat{x}}{k}\right)\right)$

$\hat{C_{E}}>0$ if $q_{0} s_{1} k>b_{1}\left(s_{0} k-\hat{x}\right)$. from (6),

$\hat{R_{1}}=\hat{C_{E}}$

from (5),

$A_{1} \hat{x}^{2}-A_{2} \hat{x}-A_{3}=0$

where, $\quad A_{1}=\left(a_{1} v+b_{1} a_{2}\right), \quad A_{2}=a_{2} k\left(s_{0} b_{1}-s_{1} q_{0}\right)+$ $a_{1}\left(v k s_{0}-b_{1}\right)>0, A_{3}=a_{1} b_{1} s_{0} k$. The Eq. (20) will have a positive root $\hat{x}$ always.

- Existence of $\overline{E_{12}}=\left(\bar{x}, \bar{y}, 0, \overline{C_{E}}, \overline{C_{0}}, \overline{R_{1}}\right)$ Here, $\bar{x}, \bar{y}, \overline{C_{E}}$, $\overline{C_{0}}$ and $\overline{R_{1}}$ are the positive solutions of the system of algebraic equations: from (1),

$C_{0}=\frac{1}{s_{1}}\left[s_{0}-\frac{x}{k}-\frac{q_{1} y}{1+x}\right]=m_{1}(x, y)$

from (4) and (5),

$C_{E}=\frac{1}{a_{1}}\left[q_{0}-b_{1} m_{1}(x, y)\right]=m_{2}(x, y)$

from (6),

$\overline{R_{1}}=\overline{C_{E}}$

Now, considering two functions [from (1) to (4)],

$W_{1}(x, y)=q_{0}-\left(a_{1}+a_{2} x\right) m_{2}(x, y)+v x m_{1}(x, y)$ 


$$
\begin{aligned}
W_{2}(x, y)= & q_{0}-a_{1} C_{E}-a_{2} x C_{E}+v x C_{0} \\
& +q_{2} x\left(s_{0}-s_{1} C_{0}-x / k\right)-q_{1} y\left(r_{0}+r_{1} y\right)
\end{aligned}
$$

For existence of $\bar{x}$ and $\bar{y}$, the two isoclines,

$W_{1}(x, y)=0$

$W_{2}(x, y)=0$

must intersect. We note that

$W_{1}(0,0)=W_{2}(0,0)=\frac{b_{1} s_{0}}{s_{1}}>0$

Also, $W_{1}(x, 0)=0$ then $x$ will have one positive root (say) $\psi_{31}$, from the following quadratic equation of $x$,

$\frac{b_{1} a_{2}}{a_{1} s_{1} k} x^{2}-x\left[\frac{a_{2}}{a_{1} s_{1}}\left(b_{1} s_{0}-q_{0} s_{1}\right)-\frac{b_{1}}{s_{1} k}\right]-\frac{b_{1} s_{0}}{s_{1}}=0$

if $\quad b_{1} s_{0}>q_{0} s_{1}$ and $k a_{2}\left(b_{1} s_{0}-q_{0} s_{1}\right)-b_{1} a_{1}>0$. $W_{1}(0, y)=0$ then $y=\frac{s_{0}}{q_{1}}=\psi_{32}$ (say). $W_{2}(x, 0)=0$ then $x$ will have one positive root (say) $\psi_{33}$, from the following quadratic equation of $x$,

$$
\begin{aligned}
& x^{2}\left(\frac{a_{2}}{a_{1} k}+\frac{v}{s_{1}}\right)-\frac{x}{a_{1} s_{1} k}\left[k\left(s_{0}\left(b_{1} a_{2}+a_{1} v\right)-a_{2} q_{0} s_{1}\right)\right. \\
& \left.-s_{1} a_{1}\right]-b_{1} s_{0} / s_{1}=0
\end{aligned}
$$

if $k\left(s_{0}\left(b_{1} a_{2}+a_{1} v\right)-a_{2} q_{0} s_{1}\right)-s_{1} a_{1}>0 . W_{2}(0, y)=0$ then $y$ will have one positive root (say) $\psi_{34}$, from the following quadratic equation of $y$,

$r_{1} q_{1} y^{2}+y\left(r_{0} q_{1}+b_{1} q_{1} / s_{1}\right)-s_{0} b_{1} / s_{1}=0$.

Thus both the isoclines intersect each other in the region

$G_{14}=\left\{(x, y): 0<x<\psi_{33}, 0<y<\psi_{32}\right\}$

in the following two cases: (see Fig.1)

Case $(i): \psi_{33}>\psi_{31}, \psi_{32}>\psi_{34}$
Case (ii) : $\psi_{33}<\psi_{31}, \psi_{32}<\psi_{34}$

This point of intersection will give $\bar{x}, \bar{y}$. For uniqueness of $(\bar{x}, \bar{y})$, we must have $\frac{d y}{d x}<0$ for both the curves in the region $G_{14}$. For curve (26),

$$
\begin{aligned}
\frac{d y}{d x} & =\frac{y}{1+x}-\frac{y}{x}-\frac{s_{1}(1+x)}{v q_{1} x}\left[\frac{2 v x}{s_{1} k}+\left(a_{1}+a_{2} x\right) m_{2}^{\prime}(x, y)\right. \\
& \left.+a_{2} m_{2}(x, y)-\frac{v s_{0}}{s_{1}}\right]<0
\end{aligned}
$$

and for curve (27),

$$
\frac{d y}{d x}=\frac{\eta_{1}+q_{2}\left(s_{0}-s_{1} m_{1}(x, y)-x / k\right)-\eta_{2}-\eta_{3}}{q_{1}\left[r_{0}+2 r_{1} y\right]}<0
$$

where, $\eta_{1}=v\left[x m_{1}^{\prime}(x, y)+m_{1}(x, y)\right], \quad \eta_{2}=\left(a_{1}+a_{2} x\right)$ $m_{2}^{\prime}(x, y)+a_{2} m_{2}(x, y), \quad \eta_{3}=q_{2} x\left[s_{1} m_{1}^{\prime}(x, y)+1 / k\right] . \quad$ In case (i), the absolute value of $\frac{d y}{d x}$ given by (30) is less than the absolute value of $\frac{d y}{d x}$ given by (31). For the case (ii), just the opposite is the condition. Knowing the values of $\bar{x}$ and $\bar{y} ; \overline{C_{E}}, \overline{C_{0}}$ and $\overline{R_{1}}$ can be computed from the Eqs. (21) to (23).

- Existence of $E_{13}^{*}=\left(x^{*}, y^{*}, z^{*}, C_{E}^{*}, C_{0}^{*}, R_{1}^{*}\right)$

Here, $x^{*}, y^{*}, z^{*}, C_{E}^{*}, C_{0}^{*}$ and $R_{1}^{*}$ are the positive solutions of the system of algebraic equations:

from (1),

$C_{0}=\frac{1}{s_{1}}\left[s_{0}-\frac{x}{k}-\frac{q_{1} y}{1+x}\right]=g_{1}(x, y)$

from (4) and (5),

$C_{E}=\frac{1}{a_{1}}\left[q_{0}-b_{1} g_{1}(x, y)\right]=g_{2}(x, y)$

from (2),

$z=\frac{1+y}{q_{3}}\left[\frac{q_{2} x}{1+x}-r_{0}-r_{1} y\right]=g_{3}(x, y)$

Fig. 1 Existence of equilibrium point $\overline{E_{12}}$ of Model 1
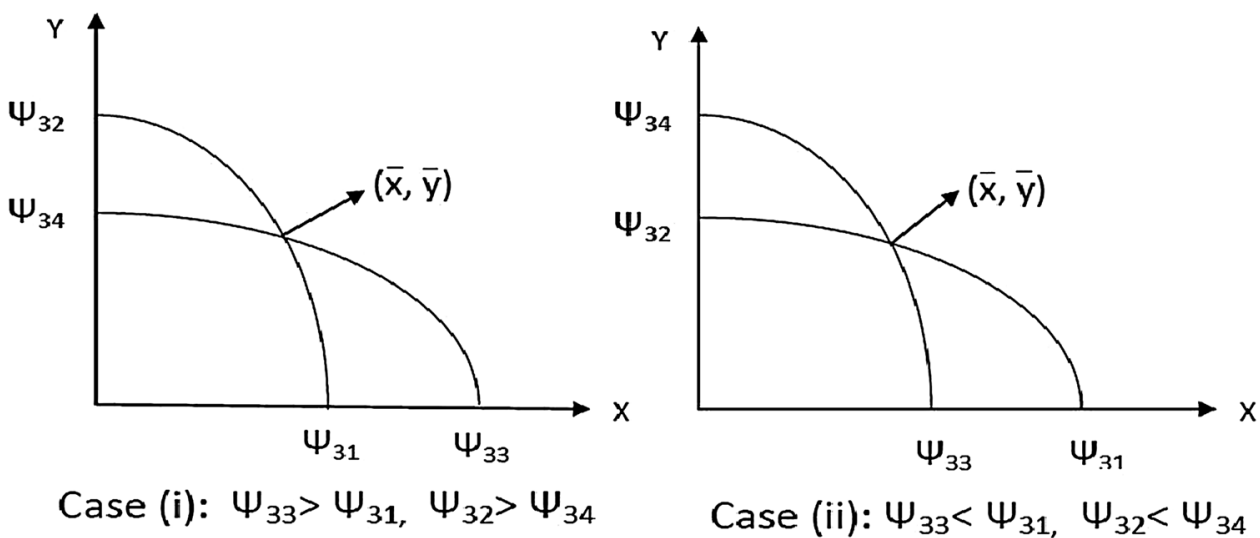

Case (ii): $\Psi_{33}<\Psi_{31}, \Psi_{32}<\Psi_{34}$ 
from (6),

$R_{1}^{*}=C_{E}^{*}$

Now, considering two functions [from (2) to (4)],

$$
\begin{aligned}
S_{11}(x, y)= & q_{0}-\left(a_{1}+a_{2} x\right) g_{2}(x, y)+v x g_{1}(x, y) \\
S_{12}(x, y)= & q_{0}-\left(a_{1}+a_{2} x\right) g_{2}(x, y)+v x g_{1}(x, y) \\
& -q_{4} y\left(r_{0}+r_{1} y\right)-q_{3} z\left(d_{1}+d_{2} z\right)+\frac{q_{2} q_{4} x y}{1+x}
\end{aligned}
$$

For existence of $x^{*}$ and $y^{*}$, the two isoclines,

$$
\begin{aligned}
& S_{11}(x, y)=0 \\
& S_{12}(x, y)=0
\end{aligned}
$$

must intersect. We note that

$$
S_{11}(0,0)=S_{12}(0,0)=\frac{b_{1} s_{0}}{s_{1}}>0
$$

Also, $S_{11}(x, 0)=0$ then $x$ will have one positive root (say) $\psi_{41}$, from the following quadratic equation of $x$,

$$
\begin{aligned}
& x^{2}\left(v+b_{1} a_{2} / a_{1}\right) / s_{1} k-x\left[\left(b_{1} s_{0}-s_{1} q_{0}\right) a_{2} / s_{1} a_{1}\right. \\
& \left.\quad+\left(v s_{0} k-b_{1}\right) / s_{1} k\right]-s_{0} b_{1} / s_{1}=0
\end{aligned}
$$

if $b_{1} s_{0}>s_{1} q_{0}$ and $v s_{0} k>b_{1} . S_{11}(0, y)=0$ then $y=\frac{s_{0}}{q_{1}}=$ $\psi_{42}$ (say).

$S_{12}(x, 0)=0$ then $x=$ will have one positive root (say) $\psi_{43}$, from the following quadratic equation of $x$,

$x^{3}\left(v+\frac{b_{1} a_{2}}{a_{1}}\right) \frac{q_{3}}{s_{1} k}-x^{2}\left[\left(b_{1} s_{0}-q_{0} s_{1}\right) \frac{a_{2} q_{3}}{a_{1} s_{1}}+\left(v s_{0} k-b_{1}\right) \frac{q_{3}}{s_{1} k}-\right.$ $\left.\left(b_{1} a_{2}+v a_{1}\right) \frac{q_{3}}{k s_{1} a_{1}}\right] \quad-x\left[\left(v+b_{1}\right) \frac{s_{0} q_{3}}{s_{1}} \quad+\left(b_{1} s_{0}-q_{0} s_{1}\right) \frac{a_{2} q_{3}}{a_{1} s_{1}}-\right.$ $\left.\left(q_{2}-r_{0}+\frac{b_{1} q_{3}}{s_{1} k}\right)\right]-\left(r_{0}+\frac{b_{1} s_{0} q_{0}}{s_{1}}\right)=0$.

if $\left(b_{1} s_{0}-q_{0} s_{1}\right) \frac{a_{2} q_{3}}{a_{1} s_{1}}+\left(v s_{0} k-b_{1}\right) \frac{q_{3}}{s_{1} k}-\left(b_{1} a_{2}+v a_{1}\right) \frac{q_{3}}{k s_{1} a_{1}}$ $>0$

and $\left[\left(v+b_{1}\right) \frac{s_{0} q_{3}}{s_{1}}+\left(b_{1} s_{0}-q_{0} s_{1}\right) \frac{a_{2} q_{3}}{a_{1} s_{1}}-\left(q_{2}-r_{0}+\frac{b_{1} q_{3}}{s_{1} k}\right)\right]$ $>0$.
$S_{12}(0, y)=0$ then $y$ will have one positive root (say) $\psi_{44}$, from the following fourth degree equation of $y$,

$G_{1} y^{4}+G_{2} y^{3}-G_{3} y^{2}+G_{4} y-G_{5}=0$

$G_{1}=d_{2} r_{1}^{2} / q_{3}>0, \quad G_{2}=2 r_{1} d_{2}\left(r_{1}+r_{0}\right) / q_{3}, \quad G_{3}=d_{1} r_{1}-$ $\left[2 r_{0} r_{1}+\left(r_{1}+r_{0}\right)^{2} d_{2} / q_{3}\right]-r_{1} q_{4}, \quad G_{4}=b_{1} q_{1} / s_{1}+r_{0} q_{4}-$ $d_{1}\left(r_{1}+r_{0}\right)+\left[2 r_{0}\left(r_{0}+r_{1}\right)\right] d_{2} / q_{3}>0$ and $G_{5}=b_{1} s_{0} / s_{1}+$ $d_{1} r_{0}-d_{2} r_{0}^{2} / q_{3}>0$

if $d_{1} r_{1}-\left[2 r_{0} r_{1}+\left(r_{1}+r_{0}\right)^{2} d_{2} / q_{3}\right]-r_{1} q_{4}>0$,

$b_{1} q_{1} / s_{1}+r_{0} q_{4}-d_{1}\left(r_{1}+r_{0}\right)+\left[2 r_{0}\left(r_{0}+r_{1}\right)\right] d_{2} / q_{3}>0$ and

$b_{1} s_{0} / s_{1}+d_{1} r_{0}-d_{2} r_{0}^{2} / q_{3}>0$.

Thus both the isoclines intersect each other in the region $G_{15}=\left\{(x, y): 0<x<\psi_{43}, 0<y<\psi_{42}\right\}$

in the following two cases: (see Fig. 2)

Case $(i): \psi_{43}>\psi_{41}, \psi_{42}>\psi_{44}$

Case (ii) : $\psi_{43}<\psi_{41}, \psi_{42}<\psi_{44}$

This point of intersection will give $x^{*}, y^{*}$. For uniqueness of $\left(x^{*}, y^{*}\right)$, we must have $\frac{d y}{d x}<0$ for both the curves in the region $G_{15}$.

For curve (38),

$$
\begin{aligned}
\frac{d y}{d x}= & \frac{y}{1+x}-\frac{y}{x}-\frac{s_{1}(1+x)}{v q_{1} x} \\
& {\left[\frac{2 v x}{s_{1} k}+\left(a_{1}+a_{2} x\right) g_{2}^{\prime}(x, y)+a_{2} g_{2}(x, y)-\frac{v s_{0}}{s_{1}}\right]<0 }
\end{aligned}
$$

and for curve (39),

$$
\frac{d y}{d x}=\frac{\eta_{11}-\eta_{12}-q_{3} g_{3}^{\prime}(x, y)\left[d_{1}+2 d_{2} g_{3}(x, y)\right]+\frac{q_{2} q_{4} y}{(1+x)^{2}}}{\frac{q_{2} q_{4} x}{1+x}-q_{4}\left(r_{0}+2 r_{1} y\right)}<0
$$

Fig. 2 Existence of equilibrium point $E_{13}^{*}$ of Model 1
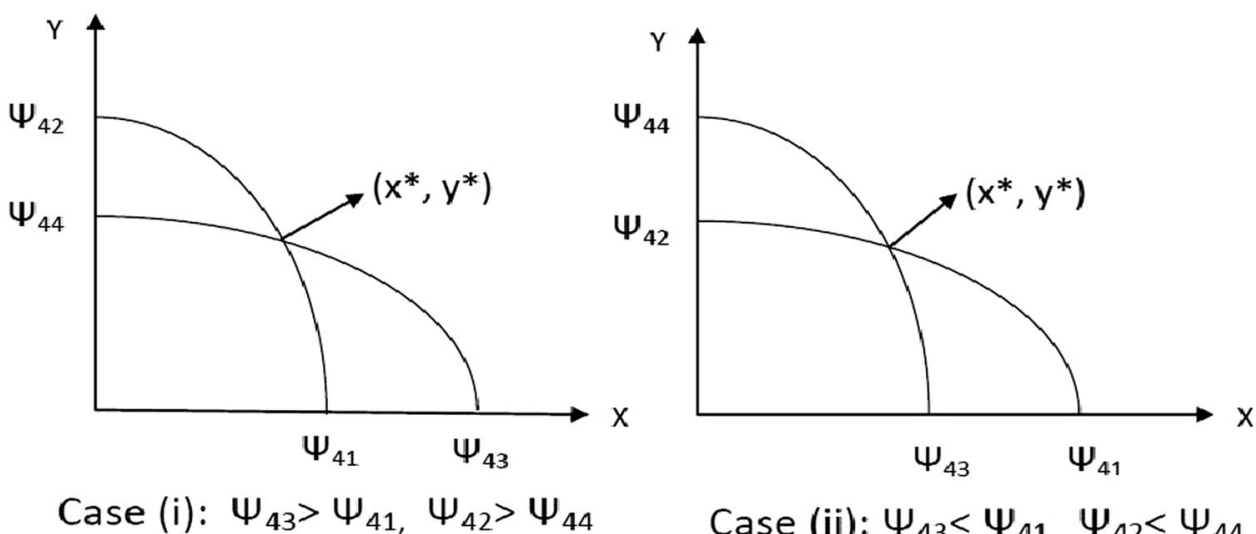

Case (ii): $\Psi_{43}<\Psi_{41}, \quad \Psi_{42}<\Psi_{44}$ 
where, $\quad \eta_{11}=v\left[x g_{1}^{\prime}(x, y)+g_{1}(x, y)\right], \quad \eta_{12}=\left(a_{1}+a_{2} x\right) g_{2}^{\prime}$ $(x, y)+a_{2} g_{2}(x, y)$. In case (i), the absolute value of $\frac{d y}{d x}$ given by (42) is less than the absolute value of $\frac{d y}{d x}$ given by (43).

For the case (ii), just the opposite is the condition.

Knowing the value of $x^{*}$ and $y^{*} ; z^{*}, C_{E}^{*}, C_{0}^{*}$ and $R_{1}^{*}$ can be computed from the Eqs. (32) to (35).

\section{Dynamical behaviour of the Model 1}

\section{Local stability}

The stability behavior of $E_{10}$ can be studied by computing variational matrix, and $\hat{E_{11}}, \overline{E_{12}}$ and $E_{13}^{*}$ can be studied by computing Liapunov's direct method.

The general variational matrix corresponding to the Model 1 is

$J\left(x, y, z, C_{E}, C_{0}, R_{1}\right)=\left[\begin{array}{cccccc}c_{11} & -c_{12} & 0 & 0 & -c_{15} & 0 \\ c_{21} & c_{22} & -c_{23} & 0 & 0 & 0 \\ 0 & c_{32} & c_{33} & 0 & 0 & 0 \\ -c_{41} & 0 & 0 & -c_{44} & c_{45} & -c_{46} \\ c_{51} & 0 & 0 & 0 & -c_{55} & c_{56} \\ 0 & 0 & 0 & c_{64} & 0 & -c_{66}\end{array}\right]$

where,

$$
\begin{gathered}
c_{11}=x\left(\frac{y q_{1}}{(1+x)^{2}}-\frac{1}{k}\right)+\left(s_{0}-s_{1} C_{0}-\frac{x}{k}-\frac{q_{1} y}{1+x}\right), \\
c_{12}=\frac{q_{1} x}{1+x}, c_{15}=s_{1} x, \\
c_{21}=\frac{q_{2} y}{(1+x)^{2}}, c_{22}=y\left(\frac{q_{3} z}{(1+y)^{2}}-r_{1}\right) \\
\quad+\left(\frac{q_{2} x}{1+x}-\frac{q_{3} z}{1+y}-r_{0}-r_{1} y\right), c_{23}=\frac{q_{3} y}{1+y}, \\
c_{32}=\frac{q_{4} z}{(1+y)^{2}}, c_{33}=\frac{q_{4} y}{1+y}-d_{1}-2 d_{2} z, \\
c_{41}=a_{2} R_{1}-v C_{0}, c_{44}=a_{1}, c_{45}=v x, \\
c_{46}=a_{2} x, c_{51}=a_{2} R_{1}-v C_{0}, c_{55}=b_{1}+v x, \\
c_{56}=a_{2} x, c_{64}=\gamma_{1}, c_{66}=\gamma_{1}
\end{gathered}
$$

About $E_{10}$, the eigenvalues of the characteristic equation are $s_{0},-r_{0},-d_{1},-a_{1},-b_{1}$ and $-\gamma_{1}$, which shows that $E_{10}$ is unstable.

About $\hat{E_{11}}$, two of the eigenvalues of the characteristic equations are $q_{2} \hat{x} /(1+\hat{x})-r_{0}$ and $-d_{1}$ and the other four eigenvalues are given by the roots of the following equation is

$$
\lambda^{4}+\lambda^{3} J_{1}+\lambda^{2} J_{2}+\lambda J_{3}+J_{4}=0
$$

where, $\quad J_{1}=\gamma_{1}+a_{1}+\left(b_{1}+v \hat{x}\right)+\hat{x} / k, J_{2}=\left(b_{1}+v \hat{x}\right)$ $\left(a_{1}+\gamma_{1}+\hat{x} / k\right)+\left(a_{1}+\gamma_{1}\right) \hat{x} / k+\left(a_{2} \hat{R_{1}}-v \hat{C_{0}}\right) \frac{q_{1} \hat{x}}{1+\hat{x}}-\gamma_{1}$ $\left(a_{2} \hat{x}-a_{1}\right)>0, J_{3}=\left(a_{1}+\gamma_{1}\right)\left(b_{1}+v \hat{x}\right) \frac{\hat{x}}{k}-\gamma_{1}\left(b_{1}+v \hat{x}\right)$ $\left(a_{2} \hat{x}-a_{1}\right)+\left(a_{2} \hat{R_{1}}-v \hat{C_{0}}\right)\left[\left(a_{1}+\gamma_{1}\right) \frac{q_{1} \hat{x}}{1+\hat{x}}-a_{2} \hat{x} \gamma_{1}\right]-\gamma_{1}\left(a_{2} \hat{x}\right.$ $\left.-a_{1}\right) \frac{\hat{x}}{k}, J_{4}=\gamma_{1}\left(a_{2} \hat{R_{1}}-v \hat{C_{0}}\right)\left[\frac{a_{1} q_{1} \hat{x}}{1+\hat{x}}-\left(2\left(\frac{q_{1} \hat{x}}{1+\hat{x}}\right)+\frac{\hat{x}}{k}\right) a_{2} \hat{x}\right]-\gamma_{1}$ $\left(b_{1}+v \hat{x}\right)\left(a_{2} \hat{x}-a_{1}\right) \hat{x}$.

According to Routh-Hurwitz criteria $\hat{E_{11}}$ is locally asymptotically stable if

$\frac{q_{2}}{r_{0}}<\frac{1}{\hat{x}}+1 \Rightarrow \frac{q_{2}}{r_{0}}<1$

$J_{i}>0(i=1,2,3,4)$,

$\frac{\hat{C_{E}}}{\hat{C_{0}}}<\frac{v}{a_{2}}, \hat{x}<\frac{a_{1}}{a_{2}}, \hat{x}<\frac{a_{1}}{2 a_{2}}, a_{2} \hat{x}(1+\hat{x})<q_{1} k\left(a_{1}-2 a_{2} \hat{x}\right)$

and $J_{1} J_{2} J_{3}>J_{3}^{2}+J_{1}^{2} J_{4}$, i.e.,

$\left(\gamma_{1}+a_{1}+\left(b_{1}+v \hat{x}\right)+\hat{x} / k\right)\left[\left(b_{1}+v \hat{x}\right)\left(a_{1}+\gamma_{1}+\hat{x} / k\right)+\right.$ $\left.\left(a_{1}+\gamma_{1}\right) \hat{x} / k+\left(a_{2} \hat{R_{1}}-v \hat{C_{0}}\right) \frac{q_{1} \hat{x}}{1+\hat{x}}-\gamma_{1}\left(a_{2} \hat{x}-a_{1}\right)\right]\left[\left(a_{1}+\right.\right.$ $\left.\gamma_{1}\right)\left(b_{1}+v \hat{x}\right) \frac{\hat{x}}{k}-\gamma_{1} \quad\left(b_{1}+v \hat{x}\right)\left(a_{2} \hat{x}-a_{1}\right)+\left(a_{2} \hat{R_{1}}-v \hat{C_{0}}\right)$ $\left.\left[\left(a_{1}+\gamma_{1}\right) \frac{q_{1} \hat{x}}{1+\hat{x}}-a_{2} \hat{x} \gamma_{1}\right]-\gamma_{1}\left(a_{2} \hat{x}-a_{1}\right) \frac{\hat{x}}{k}\right]>\left[\left(a_{1}+\gamma_{1}\right)\left(b_{1}+\right.\right.$ $v \hat{x}) \frac{\hat{x}}{k}-\gamma_{1}\left(b_{1}+v \hat{x}\right)\left(a_{2} \hat{x}-a_{1}\right)+\left(a_{2} \hat{R_{1}}-v \hat{C_{0}}\right)\left[\left(a_{1}+\gamma_{1}\right) \frac{q_{1} \hat{x}}{1+\hat{x}}\right.$ $\left.\left.-a_{2} \hat{x} \gamma_{1}\right]-\gamma_{1}\left(a_{2} \hat{x}-a_{1}\right) \frac{\hat{x}}{k}\right]^{2}+\left(\gamma_{1}+a_{1}+\left(b_{1}+v \hat{x}\right)+\hat{x} / k\right)^{2}$ $\left[\gamma_{1}\left(a_{2} \hat{R_{1}}-v \hat{C_{0}}\right)\left[\frac{a_{1} q_{1} \hat{x}}{1+\hat{x}}-\left(2\left(\frac{q_{1} \hat{x}}{1+\hat{x}}\right)+\frac{\hat{x}}{k}\right) a_{2} \hat{x}\right]-\gamma_{1}\left(b_{1}+v \hat{x}\right)\left(a_{2} \hat{x}\right.\right.$ $\left.\left.-a_{1}\right) \frac{\hat{x}}{k}\right]$.

Remark 1 From the stability conditions of $\hat{E_{11}}$, the following may be noted:

(i) the ratio of the growth rate of intermediate predator and its death rate is less than one,

(ii) the ratio of the concentration of environmental toxicant and the concentration of organismal toxicant is less than the ratio of the removal rate of toxicant from the prey species and the toxicant uptake by the prey species,

(iii) the equilibria of prey is less than the ratio of toxicant wash out from the environment and removal rate of toxicant from the environment due to uptake of by the prey species, to the ratio of toxicant wash out from the environment and the twice of removal rate of toxicant from the environment due to uptake of by the prey species, are satisfied then only prey population will survive in the presence of toxicant and when the distributed delay is considered.

Theorem 2 If the following inequalities hold

$$
q_{1} k \bar{y}<(1+\bar{x})^{2}
$$

$q_{4} \bar{y}<d_{1}(1+\bar{x})$

$9\left[s_{1} \bar{x}+T_{4}\left(v \overline{C_{0}}-a_{2} \overline{R_{1}}\right)\right]^{2}<\bar{x} T_{4}\left(b_{1}+v \bar{x}\right)\left(\frac{1}{k}-\frac{q_{1} \bar{y}}{(1+\bar{x})^{2}}\right)$

$6 T_{4}\left(a_{2} \bar{x}\right)^{2}<\gamma_{1} T_{5}\left(b_{1}+v \bar{x}\right)$ 
where,

$$
\begin{aligned}
& T_{1}=\frac{q_{1} \bar{x}(1+\bar{x})}{q_{2} \bar{y}}>0 \\
& T_{2}>\frac{2 \bar{x} q_{1}(1+\bar{x})\left(\frac{q_{3}}{1+\bar{y}}\right)^{2}}{r_{1} q_{2}\left(\frac{q_{4} \bar{y}}{1+\bar{y}}-d_{1}\right)} \\
& T_{3}<\frac{a_{1} \bar{x}}{9\left(v \overline{C_{0}}-a_{2} \overline{R_{1}}\right)^{2}}\left(\frac{1}{k}-\frac{q_{1} \bar{y}}{(1+\bar{x})^{2}}\right) \\
& T_{4}>\frac{v^{2} \bar{x}^{3}}{\left(b_{1}+v \bar{x}\right)\left(v \overline{C_{0}}-a_{2} \overline{R_{1}}\right)^{2}}\left(\frac{1}{k}-\frac{q_{1} \bar{y}}{(1+\bar{x})^{2}}\right) \\
& T_{5}<\frac{a_{1}^{2} \bar{x}}{54 \gamma_{1}\left(v \overline{C_{0}}-a_{2} \overline{R_{1}}\right)^{2}}\left(\frac{1}{k}-\frac{q_{1} \bar{y}}{(1+\bar{x})^{2}}\right)
\end{aligned}
$$

then the equilibrium $\overline{E_{12}}$ is locally asymptotically stable.

Proof We first linearize the system about the equilibrium $\overline{E_{12}}$ by using the following transformations

$$
\begin{gathered}
x=\bar{x}+n_{1} ; \quad y=\bar{y}+n_{2} ; \quad z=\bar{z}+n_{3} ; \quad C_{E}=\overline{C_{E}}+n_{4} ; \\
C_{0}=\overline{C_{0}}+n_{5} ; \quad R_{1}=\overline{R_{1}}+n_{6} ;
\end{gathered}
$$

where $n_{1}, n_{2}, n_{3}, n_{4}, n_{5}$ and $n_{6}$ are small perturbations around $\overline{E_{12}}$. Then we get the following linearized the system,

$$
\begin{aligned}
\frac{d n_{1}}{d t} & =-n_{1} \bar{x}\left(\frac{1}{k}-\frac{q_{1} \bar{y}}{(1+\bar{x})^{2}}\right)-n_{2}\left(\frac{q_{1} \bar{x}}{1+\bar{x}}\right)-n_{5} s_{1} \bar{x} \\
\frac{d n_{2}}{d t} & =n_{1}\left(\frac{q_{2} \bar{y}}{(1+\bar{y})^{2}}\right)-n_{2} r_{1} \bar{y}-n_{3}\left(\frac{q_{3} \bar{y}}{1+\bar{y}}\right) \\
\frac{d n_{3}}{d t} & =-n_{3}\left(d_{1}-\frac{q_{4} \bar{y}}{1+\bar{y}}\right) \\
\frac{d n_{4}}{d t} & =n_{1}\left(v \overline{C_{0}}-a_{2} \overline{R_{1}}\right)-a_{1} n_{4}+v \bar{x} n_{5}-a_{2} x^{*} n_{6} \\
\frac{d n_{5}}{d t} & =-n_{1}\left(v \overline{C_{0}}-a_{2} \overline{R_{1}}\right)-n_{5}\left(b_{1}+v \bar{x}\right)+n_{6} a_{2} \bar{x} \\
\frac{d n_{6}}{d t} & =n_{4} \gamma_{1}-n_{6} \gamma_{1}
\end{aligned}
$$

Now consider the following positive definite function

$$
\begin{aligned}
& V_{32}=\frac{1}{2} n_{1}^{2}+T_{1} \frac{1}{2} n_{2}^{2}+T_{2} \frac{1}{2} n_{3}^{2}+T_{3} \frac{1}{2} n_{4}^{2}+T_{4} \frac{1}{2} n_{5}^{2}++T_{5} \frac{1}{2} n_{6}^{2} \\
& \frac{d V_{32}}{d t}=n_{1} \frac{d n_{1}}{d t}+T_{1} n_{2} \frac{d n_{2}}{d t}+T_{2} n_{3} \frac{d n_{3}}{d t}+T_{3} n_{4} \frac{d n_{4}}{d t} \\
& +T_{4} n_{5} \frac{d n_{5}}{d t}+T_{5} n_{6} \frac{d n_{6}}{d t}
\end{aligned}
$$

$$
\begin{aligned}
\frac{d V_{32}}{d t}= & -n_{1}^{2} \bar{x}\left(\frac{1}{k}-\frac{q_{1} \bar{y}}{(1+\bar{x})^{2}}\right)-n_{2}^{2} r_{1} T_{1} \bar{y} \\
& -n_{3}^{2} T_{2}\left(d_{1}-\frac{q_{4} \bar{y}}{1+\bar{y}}\right)-n_{4}^{2} T_{3} a_{1} \\
- & n_{5}^{2} T_{4}\left(b_{1}+v \bar{x}\right)-n_{6}^{2} \gamma_{1} T_{5}-n_{1} n_{2}\left(\frac{q_{1} \bar{x}}{1+\bar{x}}-\frac{T_{1} q_{2} \bar{y}}{(1+\bar{x})^{2}}\right) \\
& -n_{1} n_{4} T_{3}\left(v \overline{C_{0}}-a_{2} \overline{R_{1}}\right) \\
- & n_{1} n_{5}\left(s_{1} \bar{x}+T_{4}\left(v \overline{C_{0}}-a_{2} \overline{R_{1}}\right)\right)-n_{2} n_{3} \frac{q_{3} \bar{y} T_{1}}{1+\bar{y}}+n_{4} n_{5} v \bar{x} T_{3} \\
+ & n_{4} n_{6} \gamma_{1} T_{5}+n_{5} n_{6} a_{2} \bar{x} T_{4}
\end{aligned}
$$

Now using the sylvester's criterion in the quadratic forms

$$
\begin{aligned}
\frac{d V_{32}}{d t} \leq & -\left[\left(\left(t_{11} / 2\right) n_{1}^{2}+t_{12} n_{1} n_{2}+\left(t_{22} / 2\right) n_{2}^{2}\right)\right. \\
& +\left(\left(t_{11} / 2\right) n_{1}^{2}-t_{14} n_{1} n_{4}+\left(t_{44} / 2\right) n_{4}^{2}\right) \\
& +\left(\left(t_{11} / 2\right) n_{1}^{2}+t_{15} n_{1} n_{5}+\left(t_{55} / 2\right) n_{5}^{2}\right) \\
& +\left(\left(t_{22} / 2\right) n_{2}^{2}+t_{23} n_{2} n_{3}+\left(t_{33} / 2\right) n_{3}^{2}\right) \\
& +\left(\left(t_{44} / 2\right) n_{2}^{2}-t_{45} n_{4} n_{5}+\left(t_{55} / 2\right) n_{5}^{2}\right) \\
& +\left(\left(t_{44} / 2\right) n_{2}^{2}-t_{46} n_{4} n_{6}+\left(t_{66} / 2\right) n_{3}^{2}\right) \\
& \left.+\left(\left(t_{55} / 2\right) n_{2}^{2}-t_{56} n_{2} n_{6}+\left(t_{66} / 2\right) n_{6}^{2}\right)\right]
\end{aligned}
$$

where,

$$
\begin{aligned}
t_{11}= & \left(\frac{1}{k}-\frac{q_{1} \bar{y}}{(1+\bar{x})^{2}}\right) \bar{x} / 3, t_{12}=\frac{q_{1} \bar{x}}{1+\bar{x}}-\frac{T_{1} q_{2} \bar{y}}{(1+\bar{x})^{2}}, \\
& t_{14}=T_{3}\left(v \overline{C_{0}}-a_{2} \overline{R_{1}}\right), \\
t_{15}= & s_{1} \bar{x}+T_{4}\left(v \overline{C_{0}}-a_{2} \overline{R_{1}}\right), t_{22}=r_{1} T_{1} \bar{y} / 2, t_{23}=\frac{q_{3} \bar{y} T_{1}}{1+\bar{y}}, \\
& t_{33}=T_{2}\left(d_{1}-\frac{q_{4} \bar{y}}{1+\bar{y}}\right), \\
t_{44}= & a_{1} T_{3} / 3, t_{45}=v \bar{x} T_{3}, t_{46}=\gamma_{1} T_{5}, t_{55}=\left(b_{1}+v \bar{x}\right) T_{4} / 3, \\
& t_{56}=a_{2} \bar{x} T_{4}, \\
t_{66}= & \gamma_{1} T_{5} / 2 .
\end{aligned}
$$

Sufficient conditions for $d V_{32} / d t$ to be negative definite are that the following inequalities hold:

$t_{11}>0$

$t_{33}>0$

$t_{11} t_{22}>t_{12}^{2}$

$t_{11} t_{44}>t_{14}^{2}$

$t_{11} t_{55}>t_{15}^{2}$

$t_{22} t_{33}>t_{23}^{2}$ 
$t_{44} t_{55}>t_{45}^{2}$

$t_{44} t_{66}>t_{46}^{2}$

$t_{55} t_{66}>t_{56}^{2}$

We note that the third, forth, sixth, seventh and eighth inequalities, i.e., $t_{11} t_{22}>t_{12}^{2}, t_{11} t_{44}>t_{14}^{2}, t_{22} t_{33}>t_{23}^{2}$, $t_{44} t_{55}>t_{45}^{2}$ and $t_{44} t_{66}>t_{46}^{2}$ are satisfied due to the proper choice of $T_{1}, T_{2}, T_{3}, T_{4}$ and $T_{5}$ and other inequalities, (45) $\Rightarrow(56),(46) \Rightarrow(57),(47) \Rightarrow(60)$ and $(48) \Rightarrow(64)$. Hence $V_{32}$ is a Liapunov function with respect to $\overline{E_{12}}$, proving the theorem.

Theorem 3 If the following inequalities hold

$$
\begin{aligned}
& q_{1} k y^{*}<\left(1+x^{*}\right)^{2} \\
& q_{3} z^{*}<r_{1}\left(1+y^{*}\right)^{2} \\
& 9\left(s_{1} x^{*}+L_{4}\left(v C_{0}^{*}-a_{2} R_{1}^{*}\right)\right)^{2} \\
& \quad<x^{*} L_{4}\left(b_{1}+v x^{*}\right)\left(\frac{1}{k}-\frac{q_{1} y^{*}}{\left(1+x^{*}\right)^{2}}\right) \\
& 9\left(v x^{*} L_{5}\right)^{2}<a_{1} L_{3} L_{4}\left(b_{1}+v x^{*}\right)
\end{aligned}
$$

where,

$$
\begin{gathered}
L_{1}=\frac{q_{1} x^{*}\left(1+x^{*}\right)}{q_{2} y^{*}}>0 \\
L_{2}=\frac{q_{1} q_{3} x^{*}}{q_{2} q_{4} z^{*}}\left(1+x^{*}\right)\left(1+y^{*}\right)>0 \\
L_{3}<\frac{x^{*} a_{1}}{9\left(v C_{0}^{*}-a_{2} R_{1}^{*}\right)}\left(\frac{1}{k}-\frac{q_{1} y^{*}}{\left(1+x^{*}\right)^{2}}\right) \\
L_{4}<\frac{\gamma_{1} L 5\left(b_{1}+v x^{*}\right)}{6 a_{2}^{2} x^{* 2}}>0 \\
L_{5}>\frac{a_{1} a_{2} x^{* 2}}{9 \gamma_{1}\left(v C_{0}^{*}-a_{2} R_{1}^{*}\right)}\left(\frac{1}{k}-\frac{q_{1} y^{*}}{\left(1+x^{*}\right)^{2}}\right)
\end{gathered}
$$

then the positive equilibrium $E_{13}^{*}$ is locally asymptotically stable.

Proof We first linearize the system about the equilibrium $E_{13}^{*}$ by using the following transformations

$$
\begin{aligned}
x & =x^{*}+n_{1} ; \quad y=y^{*}+n_{2} ; \quad z=z^{*}+n_{3} ; \quad C_{E}=C_{E}^{*}+n_{4} ; \\
C_{0} & =C_{0}^{*}+n_{5} ; \quad R_{1}=R_{1}^{*}+n_{6} ;
\end{aligned}
$$

where $n_{1}, n_{2}, n_{3}, n_{4}, n_{5}$ and $n_{6}$ are small perturbations around $E_{13}^{*}$. Then we get the following linearized the system,

$$
\begin{aligned}
& \frac{d n_{1}}{d t}=-n_{1} x^{*}\left(\frac{1}{k}-\frac{q_{1} y^{*}}{\left(1+x^{*}\right)^{2}}\right)-n_{2} \frac{q_{1} x^{*}}{1+x^{*}}-n_{5} s_{1} x^{*} \\
& \frac{d n_{2}}{d t}=n_{1} \frac{q_{2} y^{*}}{\left(1+x^{*}\right)^{2}}-n_{2} y^{*}\left(r_{1}-\frac{q_{3} z^{*}}{\left(1+y^{*}\right)^{2}}\right)-n_{3} \frac{q_{3} y^{*}}{1+y^{*}} \\
& \frac{d n_{3}}{d t}=n_{2} \frac{q_{4} z^{*}}{\left(1+y^{*}\right)^{2}}-n_{3} d_{2} z^{*} \\
& \frac{d n_{4}}{d t}=n_{1}\left(v C_{0}^{*}-a_{2} R_{1}^{*}\right)-a_{1} n_{4}+v x^{*} n_{5}-a_{2} x^{*} n_{6} \\
& \frac{d n_{5}}{d t}=-n_{1}\left(v C_{0}^{*}-a_{2} R_{1}^{*}\right)-n_{5}\left(b_{1}+v x^{*}\right)+n_{6} a_{2} x^{*} \\
& \frac{d n_{6}}{d t}=n_{4} \gamma_{1}-n_{6} \gamma_{1}
\end{aligned}
$$

Now consider the following positive definite function

$$
\begin{aligned}
V_{33}= & \frac{1}{2} n_{1}^{2}+L_{1} \frac{1}{2} n_{2}^{2}+L_{2} \frac{1}{2} n_{3}^{2}+L_{3} \frac{1}{2} n_{4}^{2}+L_{4} \frac{1}{2} n_{5}^{2}++L_{5} \frac{1}{2} n_{6}^{2} \\
\frac{d V_{33}}{d t}= & n_{1} \frac{d n_{1}}{d t}+L_{1} n_{2} \frac{d n_{2}}{d t}+L_{2} n_{3} \frac{d n_{3}}{d t}+L_{3} n_{4} \frac{d n_{4}}{d t} \\
& +L_{4} n_{5} \frac{d n_{5}}{d t}+L_{5} n_{6} \frac{d n_{6}}{d t} \\
\frac{d V_{33}}{d t}= & -n_{1}^{2} x^{*}\left(\frac{1}{k}-\frac{q_{1} y^{*}}{\left(1+x^{*}\right)^{2}}\right) \\
& -n_{2}^{2} L_{1} y^{*}\left(r_{1}-\frac{q_{3} z^{*}}{\left(1+y^{*}\right)^{2}}\right)-n_{3}^{2} L_{2} d_{2} z^{*} \\
- & n_{4}^{2} L_{3} a_{1}-n_{5}^{2} L_{4}\left(b_{1}+v x^{*}\right)-n_{6}^{2} \gamma_{1} L_{5} \\
& -n_{1} n_{2}\left(\frac{q_{1} x^{*}}{1+x^{*}}-\frac{L_{1} q_{2} y^{*}}{\left(1+x^{*}\right)^{2}}\right) \\
+ & n_{1} n_{4} L_{3}\left(v C_{0}^{*}-a_{2} R_{1}^{*}\right)-n_{1} n_{5}\left(s_{1} x^{*}+L_{4}\left(v C_{0}^{*}-a_{2} R_{1}^{*}\right)\right) \\
- & n_{2} n_{3}\left(\frac{L_{1} q_{3} y^{*}}{1+y^{*}}-\frac{L_{2} q_{4} z^{*}}{\left(1+y^{*}\right)^{2}}\right)+n_{4} n_{5} L_{3} v x^{*} \\
& -n_{4} n_{6}\left(a_{2} x^{*} L_{3}-L_{5} \gamma_{1}\right)+n_{5} n_{6} a_{2} x^{*} L_{4}
\end{aligned}
$$

Now using the sylvester's criterion in the quadratic forms

$$
\begin{aligned}
\frac{d V_{33}}{d t} \leq & -\left[\left(\left(e_{11} / 2\right) n_{1}^{2}+e_{12} n_{1} n_{2}+\left(e_{22} / 2\right) n_{2}^{2}\right)\right. \\
& +\left(\left(e_{11} / 2\right) n_{1}^{2}-e_{14} n_{1} n_{4}+\left(e_{44} / 2\right) n_{4}^{2}\right) \\
& +\left(\left(e_{11} / 2\right) n_{1}^{2}+e_{15} n_{1} n_{5}+\left(e_{55} / 2\right) n_{5}^{2}\right) \\
& +\left(\left(e_{22} / 2\right) n_{2}^{2}+e_{23} n_{2} n_{3}+\left(e_{33} / 2\right) n_{3}^{2}\right) \\
& +\left(\left(e_{44} / 2\right) n_{4}^{2}-e_{45} n_{4} n_{5}+\left(e_{55} / 2\right) n_{5}^{2}\right) \\
& +\left(\left(e_{44} / 2\right) n_{4}^{2}+e_{46} n_{4} n_{6}+\left(e_{66} / 2\right) n_{6}^{2}\right) \\
& \left.+\left(\left(e_{55} / 2\right) n_{5}^{2}-e_{56} n_{5} n_{6}+\left(e_{66} / 2\right) n_{6}^{2}\right)\right]
\end{aligned}
$$


where,

$$
\begin{aligned}
e_{11}= & \left(\frac{1}{k}-\frac{q_{1} y^{*}}{\left(1+x^{*}\right)^{2}}\right) x^{*} / 3, e_{12} \\
& =\left(\frac{q_{1} x^{*}}{1+x^{*}}-\frac{L_{1} q_{2} y^{*}}{\left(1+x^{*}\right)^{2}}\right), e_{14}=L_{3}\left(v C_{0}^{*}-a_{2} R_{1}^{*}\right), \\
e_{15}= & \left(s_{1} x^{*}+L_{4}\left(v C_{0}^{*}-a_{2} R_{1}^{*}\right)\right), e_{22}=L_{1}\left(r_{1}-\frac{q_{3} z^{*}}{\left(1+y^{*}\right)^{2}}\right) y^{*} / 2, \\
e_{23}= & \frac{L_{1} q_{3} y^{*}}{1+y^{*}}-\frac{L_{2} q_{4} z^{*}}{\left(1+y^{*}\right)^{2}}, e_{33}=L_{2} d_{2} z^{*}, e_{44}=a_{1} L_{3} / 3, \\
e_{45}= & L_{3} v x^{*}, e_{46}=a_{2} x^{*} L_{3}-\gamma_{1} L_{5}, e_{55}=L_{4}\left(b_{1}+v x^{*}\right) / 3, \\
& e_{56}=a_{2} x^{*} L_{4}, e_{66}=\gamma_{1} L_{5} / 2 .
\end{aligned}
$$

Sufficient conditions for $d V_{33} / d t$ to be negative definite are that the following inequalities hold:

$$
\begin{aligned}
& e_{11}>0 \\
& e_{22}>0 \\
& e_{11} e_{22}>e_{12}^{2} \\
& e_{11} e_{44}>e_{14}^{2} \\
& e_{11} e_{55}>e_{15}^{2} \\
& e_{22} e_{33}>e_{23}^{2} \\
& e_{44} e_{55}>e_{45}^{2} \\
& e_{44} e_{66}>e_{46}^{2} \\
& e_{55} e_{66}>e_{56}^{2}
\end{aligned}
$$

We note that the third, fourth, sixth, eighth and ninth inequalities, i.e., $e_{11} e_{22}>e_{12}^{2}, e_{11} e_{44}>e_{14}^{2}, e_{22} e_{33}>e_{23}^{2}$, $e_{44} e_{66}>e_{46}^{2}$ and $e_{55} e_{66}>e_{56}^{2}$ are satisfied due to the proper choice of $L_{1}, L_{2}, L_{3}, L_{4}$ and $L_{5}$ and other inequalities, (65) $\Rightarrow(69),(66) \Rightarrow(70),(67) \Rightarrow(73)$ and $(68) \Rightarrow(75)$. Hence $V_{33}$ is a Liapunov function with respect to $E_{13}^{*}$, proving the theorem.

\section{Global stability}

Theorem $4 E_{13}^{*}$ is globally asymptotically stable if $q_{1}^{*}>q_{1}, q_{3}^{*}>q_{3}, a_{1}>a_{1}^{*}, b_{1}>b_{1}^{*}, v<v^{*}, a_{2}^{*}>a_{2}$ and $\gamma_{1}>\gamma_{1}^{*}$, where, $q_{1}^{*}, q_{3}^{*}, a_{1}^{*}, b_{1}^{*}, v^{*}, a_{2}^{*}$ and $\gamma_{1}^{*}$ are given in proof.

Proof We first linearize the system about the equilibrium $E_{13}^{*}$ by using the following transformations

$$
\begin{aligned}
x & =x^{*}+n_{1} ; \quad y=y^{*}+n_{2} ; \quad z=z^{*}+n_{3} ; C_{E}=C_{E}^{*}+n_{4} ; \\
C_{0} & =C_{0}^{*}+n_{5} ; \quad R_{1}=R_{1}^{*}+n_{6} ;
\end{aligned}
$$

where $n_{1}, n_{2}, n_{3}, n_{4}, n_{5}$ and $n_{6}$ are small perturbations around $E_{13}^{*}$. Then we get the following linearized the system,

$$
\begin{aligned}
\frac{d n_{1}}{d t} & =\left(x^{*}+n_{1}\right)\left[-n_{1}\left(\frac{1}{k}-\frac{q_{1} y^{*}}{\pi_{31}}\right)-n_{2} \frac{q_{1}\left(1+x^{*}\right)}{\pi_{31}}-n_{5} s_{1}\right] \\
\frac{d n_{2}}{d t} & =\left(y^{*}+n_{2}\right)\left[\frac{q_{2} n_{1}}{\pi_{31}}-n_{2}\left(r_{1}-\frac{q_{3} z^{*}}{\pi_{32}}\right)-n_{3} \frac{q_{3}\left(1+y^{*}\right)}{\pi_{32}}\right] \\
\frac{d n_{3}}{d t} & =\left(z^{*}+n_{3}\right)\left[n_{2} \frac{q_{4}}{\pi_{32}}-n_{3} d_{2}\right] \\
\frac{d n_{4}}{d t} & =n_{1}\left(v C_{0}^{*}-a_{2} R_{1}^{*}\right)-a_{1} n_{4}+v x n_{5}-a_{2} x n_{6} \\
\frac{d n_{5}}{d t} & =-n_{1}\left(v C_{0}^{*}-a_{2} R_{1}^{*}\right)-n_{5}\left(b_{1}+v x\right)+n_{6} a_{2} x \\
\frac{d n_{6}}{d t} & =n_{4} \gamma_{1}-n_{6} \gamma_{1}
\end{aligned}
$$

Where, $\quad \pi_{31}=(1+x)\left(1+x^{*}\right), \quad \pi_{32}=(1+y)\left(1+y^{*}\right)$. Now, we consider the following positive definite function:

$$
\begin{aligned}
V_{34}= & \left\{n_{1}-x^{*} \log \left(1+\frac{n_{1}}{x^{*}}\right)\right\}+F_{1}\left\{n_{2}-y^{*} \log \left(1+\frac{n_{2}}{y^{*}}\right)\right\} \\
& +F_{2}\left\{n_{3}-z^{*} \log \left(1+\frac{n_{3}}{z^{*}}\right)\right\}+\frac{F_{3}}{2} n_{4}^{2}+\frac{F_{4}}{2} n_{5}^{2}+\frac{F_{5}}{2} n_{6}^{2} \\
\frac{d V_{34}}{d t}= & \frac{n_{1}}{x^{*}+n_{1}} \frac{d n_{1}}{d t}+\frac{n_{2} F_{1}}{y^{*}+n_{2}} \frac{d n_{2}}{d t}+\frac{n_{3} F_{2}}{z^{*}+n_{3}} \frac{d n_{3}}{d t}+F_{3} n_{4} \frac{d n_{4}}{d t} \\
& +F_{4} n_{5} \frac{d n_{5}}{d t}+F_{5} n_{6} \frac{d n_{6}}{d t} \\
\frac{d V_{34}}{d t}= & -n_{1}^{2} x^{*}\left(\frac{1}{k}-\frac{q_{1} y^{*}}{\pi_{31}}\right)-n_{2}^{2} F_{1}\left(r_{1}-\frac{q_{3} z^{*}}{\pi_{32}}\right)-n_{3}^{2} F_{2} d_{2} \\
& -n_{4}^{2} a_{1}-n_{5}^{2}\left(b_{1}+v x\right)-n_{6}^{2} \gamma_{1}-n_{1} n_{2}\left(q_{1}\left(1+x^{*}\right)\right. \\
& \left.-F_{1} q_{2}\right) / \pi_{31}+n_{1} n_{4}\left(v C_{0}^{*}-a_{2} R_{1}^{*}\right) \\
& -n_{1} n_{5}\left(s_{1}+\left(v C_{0}^{*}-a_{2} R_{1}^{*}\right)\right)-n_{2} n_{3}\left(F_{1} q_{3}\left(1+y^{*}\right)\right. \\
& \left.-F_{2} q_{4}\right) / \pi_{32}+n_{4} n_{5} v x-n_{4} n_{6}\left(a_{2} x-\gamma_{1}\right)+n_{5} n_{6} a_{2} x
\end{aligned}
$$

Now, choosing $F_{1}=\frac{q_{1}}{q_{2}}\left(1+x^{*}\right)>0$ and $F_{2}=\frac{q_{1} q_{3}}{q_{2} q_{4}}(1+$ $\left.x^{*}\right)\left(1+y^{*}\right)>0$ from above Eq. (78). Now using the sylvester's criterion in the quadratic forms,

$$
\begin{aligned}
\frac{d V_{34}}{d t} \leq & -\left[\left(\left(f_{11} / 2\right) n_{1}^{2}+f_{14} n_{1} n_{4}+\left(f_{44} / 2\right) n_{4}^{2}\right)+\left(\left(f_{11} / 2\right) n_{1}^{2}\right.\right. \\
& \left.+f_{15} n_{1} n_{5}+\left(f_{55} / 2\right) n_{5}^{2}\right)+\left(\left(f_{44} / 2\right) n_{4}^{2}-f_{45} n_{4} n_{5}\right. \\
& \left.+\left(f_{55} / 2\right) n_{5}^{2}\right)+\left(\left(f_{44} / 2\right) n_{4}^{2}+f_{46} n_{4} n_{6}+\left(f_{66} / 2\right) n_{6}^{2}\right) \\
& +\left(\left(f_{55} / 2\right) n_{5}^{2}-f_{56} n_{5} n_{6}+\left(f_{66} / 2\right) n_{6}^{2}\right)
\end{aligned}
$$

where, $\quad f_{11}=\left(\frac{1}{k}-\frac{q_{1} y^{*}}{\pi_{31}}\right) / 2, f_{14}=v C_{0}^{*}-a_{2} R_{1}^{*}, \quad f_{15}=s_{1}+$ $\left(v C_{0}^{*}-a_{2} R_{1}^{*}\right), f_{44}=a_{1} / 3, f_{45}=v x, \quad f_{46}=a_{2} x-\gamma_{1}, f_{55}=$ $\left(b_{1}+v x\right) / 3, f_{56}=a_{2} x f_{66}=\gamma_{1} / 2$. 
Sufficient conditions for $d V_{34} / d t$ to be negative definite are that the following inequalities hold:

$$
\text { (i) } f_{11}>0, \quad\left(\text { ii) } f_{22}>0, \quad(\text { iii }) f_{11} f_{44}>f_{14}^{2}, \quad(i v) f_{11} f_{55}>f_{15}^{2},\right.
$$$$
\text { (v) } f_{44} f_{55}>f_{45}^{2}, \quad(v i) f_{44} f_{66}>f_{46}^{2}, \quad(v i i) f_{55} f_{66}>f_{56}^{2} \text {. }
$$

Clearly, by Liapunov's direct method $E_{13}^{*}$ is globally asymptotically stable. Further, the above conditions (i)(vii), obtained by using Sylvester's criteria, can be rewritten respectively in the following form:

$$
\begin{aligned}
& \left(1+x_{\max }\right)\left(1+x^{*}\right)>q_{1} k y^{*} \\
& \left(1+y_{\max }\right)\left(1+y^{*}\right) r_{1}>q_{3} z^{*} \\
& a_{1}\left(\frac{1}{k}-\frac{q_{1} y^{*}}{\left(1+x_{\max }\right)\left(1+x^{*}\right)}\right)>6\left(v C_{0}^{*}-a_{2} R_{1}^{*}\right)^{2} \\
& \left(b_{1}+v x_{\max }\right)\left(\frac{1}{k}-\frac{q_{1} y^{*}}{\left(1+x_{\max }\right)\left(1+x^{*}\right)}\right)>6\left(s_{1}+\left(v C_{0}^{*}-a_{2} R_{1}^{*}\right)\right)^{2}
\end{aligned}
$$

$$
\begin{aligned}
& a_{1}\left(b_{1}+v x_{\text {max }}\right)>6 v^{2} x_{\text {min }}^{2} \\
& a_{1} \gamma_{1}>6\left(a_{2} x_{\text {min }}-\gamma_{1}\right)^{2} \\
& \gamma_{1}\left(b_{1}+v x_{\text {max }}\right)>6 a_{2}^{2} x_{\text {min }}^{2}
\end{aligned}
$$

where $x_{\max }, y_{\max }$ and $x_{\min }$ can be obtained from region of attraction $\Omega$. Here, we have taken above inequalities in such a way that right hand side becomes minimum and the left hand side becomes maximum. This particular case represents the weakest situation for the inequalities to be held and, moreover, if inequalities are dissatisfied in this situation then the paths starting in $\Omega$ will not go to interior equilibrium point. Thus, under this particular situation we will break the above inequalities (79)-(85) to obtain the threshold conditions depending upon toxicant and predation related parameters.

Now, by some algebraic manipulation it is clear that inequality (79), holds true when $q_{1}^{*}>q_{1}$, where

$$
q_{1}^{*}=\frac{\left(1+x_{\max }\right)\left(1+x^{*}\right)}{k y^{*}}
$$

Now, by some algebraic manipulation it is clear that inequality (80), holds true when $q_{3}^{*}>q_{3}$, where

$$
q_{3}^{*}=\frac{r_{1}}{z^{*}}\left(1+y_{\max }\right)\left(1+y^{*}\right)
$$

Now, by some algebraic manipulation it is clear that inequality (81), holds true when $a_{1}>a_{1}^{*}$, where

$$
a_{1}^{*}=\frac{6\left(v C_{0}^{*}-a_{2} R_{1}^{*}\right)^{2}}{\left(\frac{1}{k}-\frac{q_{1} y^{*}}{\left(1+x_{\max }\right)\left(1+x^{*}\right)}\right)}
$$

Now, by some algebraic manipulation it is clear that inequality (82), holds true when $b_{1}>b_{1}^{*}$, where

$$
b_{1}^{*}=\frac{6\left(s_{1}+\left(v C_{0}^{*}-a_{2} R_{1}^{*}\right)\right)^{2}}{\left(\frac{1}{k}-\frac{q_{1} y^{*}}{\left(1+x_{\max }\right)\left(1+x^{*}\right)}\right)}-v x_{\max }
$$

Now, by some algebraic manipulation it is clear that inequality (83), we obtain the following result:

$F_{11}(v)=\left(\frac{6 x_{\min }^{2}}{a_{1}}\right) v^{2}-x_{\max } v-b_{1}<0$

by theory of equations there exists a positive root of $F_{11}(v)=0$, say it is $v=v^{*}$. Then, clearly the above inequality holds when $v<v^{*}$.

Now, by some algebraic manipulation it is clear that inequality (84), holds true when $a_{2}^{*}>a_{2}$, where

$$
a_{2}^{*}=\frac{1}{x_{\min }}\left(\sqrt{\frac{a_{1} \gamma_{1}}{6}}+\gamma_{1}\right)
$$

Now, by some algebraic manipulation it is clear that inequality (85), holds true when $\gamma_{1}>\gamma_{1}^{*}$, where

$\gamma_{1}^{*}=\frac{6 a_{2}^{2} x_{\min }^{2}}{b_{1}+v x_{\max }}$

Its clear that $E_{13}^{*}$ is globally asymptotically stable if $q_{1}^{*}>q_{1}, q_{3}^{*}>q_{3}, a_{1}>a_{1}^{*}, b_{1}>b_{1}^{*}, v<v^{*}, a_{2}^{*}>a_{2}$ and $\gamma_{1}>\gamma_{1}^{*}$. This completes the proof of the theorem.

\section{Analysis of Model 2}

\section{Equilibria of Model 2}

The Model 2 has four non negative equilibria in $x, y, z, C_{E}, C_{0}$

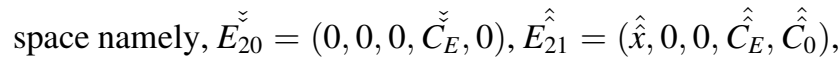
$\overline{E_{22}}=\left(\overline{\bar{x}}, \overline{\bar{y}}, 0, \overline{\overline{C_{E}}}, \overline{\overline{C_{0}}}\right)$ and $E_{23}^{* *}=\left(x^{* *}, y^{* *}, z^{* *}, C_{E}^{* *}, C_{0}^{* *}\right)$. The existence of $E_{20}$ is obvious.

Here we observe that, the equilibria of Model 2 and Model 1 have the same non-negative conditions.

\section{Dynamical behaviour of the Model 2}

\section{Local stability}

The stability behavior of $E_{20}$ can be studied by computing variational matrix, and $\hat{E_{21}}, \overline{E_{22}}$ and $E_{23}^{* *}$ can be studied by computing Liapunov's direct method.

The general variational matrix corresponding to the Model 2 is 
$J\left(x, y, z, C_{E}, C_{0}\right)=\left[\begin{array}{ccccc}n_{11} & -n_{12} & 0 & 0 & -n_{15} \\ n_{21} & n_{22} & -n_{23} & 0 & 0 \\ 0 & n_{32} & n_{33} & 0 & 0 \\ n_{41} & 0 & 0 & -n_{44} & n_{45} \\ -n_{51} & 0 & 0 & n_{54} & -n_{55}\end{array}\right]$

Remark 2 From the stability conditions of $\hat{E_{21}}$, the following may be noted:

(i) the ratio of the growth rate of intermediate predator and its death rate is less than one,

(ii) the equilibria of prey is lies between the residence time of organismal concentration in the population and the ratio of decay rate of environment toxicant concentration and the prey population due to toxicant are satisfied then only prey population will survive in the presence of toxicant.

$$
\begin{aligned}
n_{11}= & x\left(\frac{y q_{1}}{(1+x)^{2}}-\frac{1}{k}\right)+\left(s_{0}-s_{1} C_{0}-\frac{x}{k}-\frac{q_{1} y}{1+x}\right), \\
& n_{12}=\frac{q_{1} x}{1+x}, n_{15}=s_{1} x, \\
n_{21}= & \frac{q_{2} y}{(1+x)^{2}}, n_{22}=y\left(\frac{q_{3} z}{(1+y)^{2}}-r_{1}\right) \\
& +\left(\frac{q_{2} x}{1+x}-\frac{q_{3} z}{1+y}-r_{0}-r_{1} y\right), n_{23}=\frac{q_{3} y}{1+y}, \\
n_{32}= & \frac{q_{4} z}{(1+y)^{2}}, n_{33}=\frac{q_{4} y}{1+y}-d_{1}-2 d_{2} z, \\
& n_{41}=v C_{0}-a_{2} C_{E}, n_{44}=a_{1}+a_{2} x, \\
n_{45}= & v x, n_{51}=v C_{0}, n_{54}=a_{2} x, n_{55}=b_{1}+v x .
\end{aligned}
$$

About $E_{20}$, the eigenvalues of the characteristic equation are $s_{0},-r_{0},-d_{1},-a_{1}$ and $-b_{1}$, which shows that $E_{20}$ is unstable.

About $\hat{E_{21}}$, two eigenvalues of the characteristic equation are, $-d_{1}, \frac{q_{2} \hat{\hat{x}}}{1+\hat{x}}-r_{0}$ and the other three eigenvalues are given by the roots of the following equation

$$
\begin{aligned}
& \lambda^{3}+\lambda^{2} M_{1}+\lambda M_{2}+M_{3}=0 \\
& M_{1}=\hat{\hat{x}} / k+\left(a_{1}+b_{1}\right)+\hat{\hat{x}}\left(v+a_{2}\right) \text {, } \\
& M_{2}=\left[\left(a_{1}+b_{1}\right)+\hat{\hat{x}}\left(v+a_{2}\right)\right] \hat{\hat{x}} / k+a_{1} b_{1}+\hat{\hat{x}} \quad\left(b_{1} a_{2}+\right. \\
& \left.a_{1} v\right)-v s_{1} \hat{\hat{x}}^{2} \text {, } \\
& M_{3}=\left[\left(a_{1}+b_{1}\right)+\hat{\hat{x}}\left(v+a_{2}\right)\right] \hat{\hat{x}} / k-s_{1} \hat{\hat{x}}^{2}\left[v\left(a_{1}+a_{2} \hat{\hat{x}}\right)+\right. \\
& \left.\hat{\hat{C_{E}}} a_{2}^{2}+v a_{2} \hat{\hat{C_{0}}}\right] \text {. According to Routh-Hurwitz criteria } \hat{E_{21}} \text { is } \\
& \text { locally asymptotically stable if } \\
& \frac{q_{2}}{r_{0}}<\frac{1}{\hat{x}}+1 \Rightarrow \frac{q_{2}}{r_{0}}<1 \\
& M_{2}>0 \text {, i.e., }\left[\left(a_{1}+b_{1}\right)+\hat{\hat{x}}\left(v+a_{2}\right)\right] \hat{\hat{x}} / k+a_{1} b_{1}+\hat{\hat{x}}\left(b_{1} a_{2}+\right. \\
& \left.a_{1} v\right)>v s_{1} \hat{\hat{x}}^{2} \text {, } \\
& M_{3}>0 \text {, i.e., }\left[\left(a_{1}+b_{1}\right)+\hat{\hat{x}}\left(v+a_{2}\right)\right] \hat{\hat{x}} / k>s_{1} \hat{\hat{x}}^{2}\left[v \left(a_{1}+\right.\right. \\
& \left.\left.a_{2} \hat{\hat{x}}\right)+\hat{\hat{C_{E}}} a_{2}^{2}+v a_{2} \hat{\hat{C_{0}}}\right] \text { and } M_{1} M_{2}-M_{3}>0 \text {, i.e., }\left[\left(a_{1}+\right.\right. \\
& \left.\left.b_{1}\right)+\hat{\hat{x}}\left(v+a_{2}\right)+\hat{\hat{x}}\right]\left[b_{1}\left(a_{1}+\hat{\hat{x}} a_{2}\right)+v \hat{\hat{x}}\left(a_{1}-s_{2} \hat{\hat{x}}\right)\right]+s_{1} \hat{\hat{x}}^{2} \\
& {\left[v\left(a_{1}+a_{2} \hat{\hat{x}}\right)+\hat{\hat{C_{E}}} a_{2}+v a_{2} \hat{\hat{C_{0}}}\right]+\frac{\hat{\hat{x}}}{k}\left[\left(a_{1}+b_{1}\right)+\hat{\hat{x}}\left(v+a_{2}\right)\right]\left[\frac{\hat{\hat{x}}}{k}\right.} \\
& \left.+\left(a_{1}+b_{1}\right)+\left(\hat{\hat{x}}\left(v+a_{2}\right)-1\right)\right]>0 \text {, } \\
& M_{1} M_{2}-M_{3}>0 \text { if } \\
& \frac{1}{v+a_{2}}<\hat{\hat{x}}<\frac{a_{1}}{s_{1}} \text {. }
\end{aligned}
$$

\section{Remark 3}

$$
\frac{\partial \hat{\hat{x}}}{\partial \hat{\hat{C}_{0}}}=-s_{1} k<0
$$

It may be noted from Eq. (87) that the prey population will decrease with respect to increasing organismal toxicant concentration.

About $\overline{E_{22}}$, one of the eigenvalues of the characteristic equation is $q_{4} \overline{\bar{y}} /(1+\overline{\bar{y}})-d_{1}$ and the other four eigenvalues are given by the roots of the following equation is

$$
\lambda^{4}+\lambda^{3} U_{1}+\lambda^{2} U_{2}+\lambda U_{3}+U_{4}=0
$$

where, $U_{1}=p_{15}+p_{14}+r_{1} \overline{\bar{y}}-\overline{\bar{x}} p_{11}$

$$
U_{2}=p_{12} p_{13}-\overline{\bar{x}} p_{11}\left(r_{1} \overline{\bar{y}}+p_{14}\right)+r_{1} \overline{\bar{y}} p_{14}-s_{1} \overline{\bar{x}} v \overline{\overline{C_{0}}}-v a_{2}
$$$$
\overline{\bar{x}}^{2}+\left(r_{1} \overline{\bar{y}}+p_{14}-\overline{\bar{x}} p_{11}\right) p_{15}
$$$$
U_{3}=\left(p_{12} p_{13}-\overline{\bar{x}} p_{11} r_{1} \overline{\bar{y}}\right) p_{14}-s_{1} \overline{\bar{x}} v \overline{\bar{C}_{0}}\left(r_{1} \overline{\bar{y}}+p_{14}\right)+s_{1} \overline{\bar{x}}
$$$$
\left(\begin{array}{lll}
v \overline{\overline{C_{0}}} & -a_{2} & \overline{\overline{C_{E}}}
\end{array}\right) a_{2} \overline{\bar{x}}-v a_{2} \overline{\bar{x}}^{2}\left(r_{1} \overline{\bar{y}}-\overline{\bar{x}} p_{11}\right)+\left(p_{12} p_{13}-\overline{\bar{x}} p_{11}\right.
$$$$
\left.r_{1} \overline{\bar{y}}\right) p_{15}+\left(r_{1} \overline{\bar{y}}-\overline{\bar{x}} p_{11}\right) p_{14} p_{15}
$$

$$
U_{4}=s_{1} \overline{\bar{x}} r_{1} \overline{\bar{y}}\left(\left(v \overline{\overline{C_{0}}}-a_{2} \overline{\overline{C_{E}}}\right) a_{2} \overline{\bar{x}}-p_{14} v \overline{\overline{C_{0}}}\right)+\left(p_{12} p_{13}-\right.
$$
$\left.\overline{\bar{x}} p_{11} r_{1} \overline{\bar{y}}\right)\left(p_{14} p_{15}-v a_{2} \overline{\bar{x}}^{2}\right)$

$$
p_{11}=\frac{q_{1} \overline{\bar{y}}}{(1+\bar{x})^{2}}-\frac{1}{k}, p_{12}
$$$$
=\frac{q_{1} \overline{\bar{x}}}{1+\bar{x}}, p_{13}=\frac{q_{2} \overline{\bar{y}}}{(1+\overline{\bar{x}})^{2}}, p_{14}=a_{1}+a_{2} \overline{\bar{x}}, p_{15}=b_{1}+v \overline{\bar{x}} \text {. }
$$

From the Routh-Hurwitz's criteria it is found that $\overline{E_{22}}$ is locally asymptotically stable if the following conditions hold good.

$$
q_{4} \overline{\bar{y}}<d_{1}(1+\overline{\bar{y}}) \text {, }
$$

$U_{i}>0, i=1,2,3,4, U_{1} U_{2}>U_{3}$ i.e.,

$$
\left(\left(b_{1}+v \overline{\bar{x}}\right)(1-\overline{\bar{x}})+\left(r_{1} \overline{\bar{y}}-\overline{\bar{x}} p_{11}\right)\right) \frac{\overline{\bar{x}} \overline{\bar{y}} q_{1} q_{2}}{(1+\bar{x})^{3}}+\left(a_{1}+a_{2} \overline{\bar{x}}+\right.
$$
$\left.r_{1} \overline{\bar{y}}\right)\left[\left(a_{1}+a_{2} \overline{\bar{x}}\right)(1+r \overline{\bar{y}})+\left(b_{1}+v \overline{\bar{x}}\right)+r_{1} \overline{\bar{y}}-\overline{\bar{x}} p_{11}\right]-\overline{\bar{x}}\left(a_{1}+\right.$ $\left.a_{2} \overline{\bar{x}}+r_{1} \overline{\bar{y}}\right) p_{11}\left[a_{1}+b_{1}+\overline{\bar{x}}\left(a_{2}+v\right)+r_{1} \overline{\bar{y}}-\overline{\bar{x}} p_{11}\right]+\overline{\bar{x}} v s_{1} \overline{\overline{C_{0}}}$ $\left(\overline{\bar{x}} p_{11}-b_{1}\right)-\left(a_{1}+b_{1}+\overline{\bar{x}}\left(a_{2}+v\right)\right) \quad v a_{2} \overline{\bar{x}}^{2}-s_{1} \overline{\bar{x}}^{2}\left(v \overline{\bar{C}_{0}}\left(a_{2}\right.\right.$ $\left.+v)-a_{2}^{2} \overline{\overline{C_{E}}}\right)-\overline{\bar{x}}\left(b_{1}+v \overline{\bar{x}}\right)\left(b_{1}+v \overline{\bar{x}}+r_{1} \overline{\bar{y}}\right) p_{11}+\overline{\bar{x}}^{2}\left(b_{1}+v \overline{\bar{x}}\right)$ $p_{11}\left(r_{1} \overline{\bar{y}}+p_{11}\right)>0$, and $U_{1} U_{2} U_{3}>\left(U_{3}^{2}+U_{1}^{2} U_{4}\right)$, i.e., $\left(p_{14}+p_{15}+r_{1} \overline{\bar{y}}-\overline{\bar{x}} p_{11}\right)\left[p_{12} p_{13}-\overline{\bar{x}} p_{11}\left(r_{1} \overline{\bar{y}}+p_{14}\right)+r_{1} \overline{\bar{y}} p_{14}\right.$ 
$\left.-\overline{\bar{x}} v\left(s_{1} \overline{\overline{C_{0}}}+a_{2} \overline{\bar{x}}\right)+p_{15}\left(r_{1} \overline{\bar{y}}+p_{14}-\overline{\bar{x}} p_{11}\right)\right]\left[\left(p_{12} p_{13}-\overline{\bar{x}} p_{11}\right.\right.$

$\left.r_{1} \overline{\bar{y}}\right)\left(p_{14}+\overline{\bar{x}} p_{15}\right)+\left(r_{1} \overline{\bar{y}}-\overline{\bar{x}} p_{11}\right)\left(p_{14} p_{15}-v a_{2} \overline{\bar{x}}^{2}\right)-s_{1} \overline{\bar{x}} v \overline{\overline{C_{0}}}$ $\left.\left(r_{1} \overline{\bar{y}}+p_{14}\right)+s_{1} a_{2} \overline{\bar{x}}^{2} \quad\left(v \overline{\overline{C_{0}}}-a_{2} \overline{\overline{C_{E}}}\right)\right]>\left[\begin{array}{ll}\left(p_{12} p_{13}\right. & \left.-\overline{\bar{x}} p_{11} r_{1} \overline{\bar{y}}\right)\end{array}\right.$ $\left(p_{14}+\overline{\bar{x}} p_{15}\right)+\left(r_{1} \overline{\bar{y}}-\overline{\bar{x}} p_{11}\right)\left(p_{14} p_{15}-v a_{2} \overline{\bar{x}}^{2}\right)-s_{1} \overline{\bar{x}} v \overline{\bar{C}_{0}}\left(r_{1} \overline{\bar{y}}\right.$ $\left.\left.+p_{14}\right)+s_{1} a_{2} \overline{\bar{x}}^{2}\left(v \overline{\overline{C_{0}}}-a_{2} \overline{\overline{C_{E}}}\right)\right]^{2}+\left(p_{14}+p_{15}+r_{1} \overline{\bar{y}}-\overline{\bar{x}} p_{11}\right)^{2}$ $\left[\overline{\bar{x}} \overline{r_{1}} s_{1}\left(\left(v \overline{\overline{C_{0}}}-a_{2} \overline{\overline{C_{E}}}\right) a_{2} \overline{\bar{x}}\right.\right.$ $\left.\left.-v \overline{\overline{C_{0}}} p_{14}\right)+\left(p_{12} p_{13}-\overline{\bar{x}} \overline{\bar{y}} r_{1} p_{11}\right)\left(p_{14} p_{15}-v a_{2} \overline{\bar{x}}^{2}\right)\right]$.

The characteristic equation of $E_{23}^{* *}$ is

$\lambda^{5}+\lambda^{4} Q_{1}+\lambda^{3} Q_{2}+\lambda^{2} Q_{3}+\lambda Q_{4}+Q_{5}=0$

where,

$$
\begin{aligned}
& Q_{1}=\left(d_{2} z^{* *}+q_{17}+q_{18}\right)-\left(x^{* *} q_{11}+y^{* *} q_{14}\right) \\
& Q_{2}=q_{12} q_{13}+x^{* *} q_{11} y^{* *} q_{14}+q_{15} q_{16}-\left(x^{* *} q_{11}+y^{* *} q_{14}\right) d_{2} z^{* *} \\
& -\left(x^{* *} q_{11}+y^{* *} q_{14}-d_{2} z^{* *}\right) q_{17}-s_{1} v x^{* *} C_{0}^{* *} \\
& -v a_{2}\left(x^{* *}\right)^{2}+\left(d_{2} z^{* *}+q_{17}-x^{* *} q_{11}-y^{* *} q_{14}\right) q_{18} \\
& Q_{3}=\left(q_{12} q_{13}+x^{* *} q_{11} y^{* *} q_{14}\right) d_{2} z^{* *}-x^{* *} q_{11} q_{15} q_{16} \\
& +\left(q_{12} q_{13}+x^{* *} q_{11} y^{* *} q_{14}+q_{15} q_{16}\right. \\
& \left.-x^{* *} q_{11} d_{2} z^{* *}-y^{* *} q_{14} d_{2} z^{* *}\right) q_{17}+\left(y^{* *} q_{14}-d_{2} z^{* *}\right. \\
& \left.-q_{17}\right) s_{1} x^{* *} v C_{0}^{* *}+\left(s_{1} x^{* *}\left(v C_{0}^{* *}-a_{2} C_{E}^{* *}\right)+q_{11} v\left(x^{* *}\right)^{2}\right. \\
& \left.+y^{* *} q_{14} v x^{* *}-d_{2} z^{* *} v x^{* *}\right) a_{2} x^{* *}+\left(q_{12} q_{13}+x^{* *} q_{11} y^{* *} q_{14}\right. \\
& +q_{15} q_{16}-x^{* *} q_{11} d_{2} z^{* *}-y^{* *} q_{14} d_{2} z^{* *}-x^{* *} q_{11} q_{17} \\
& \left.-y^{* *} q_{14} q_{17}+d_{2} z^{* *} q_{17}\right) q_{18} \\
& Q_{4}=q_{12} q_{13} d_{2} z^{* *} q_{17}+x^{* *} q_{11} y^{* *} q_{14} d_{2} z^{* *} q_{17}-x^{* *} q_{11} q_{15} q_{16} q_{17} \\
& +\left(y^{* *} q_{14} d_{2} z^{* *}-q_{15} q_{16}\right) s_{1} x^{* *} v C_{0}^{* *} \\
& -\left(d_{2} z^{* *}-y^{* *} q_{14}\right) s_{1} x^{* *} q_{17} \nu C_{0}^{* *}+\left(d_{2} z^{* *}-y^{* *} q_{14}\right) \\
& \left(v C_{0}^{* *}-a_{2} C_{E}^{* *}\right) s_{1} a_{2}\left(x^{* *}\right)^{2}+\left(x^{* *} q_{11} d_{2} z^{* *}+y^{* *} q_{14} d_{2} z^{* *}\right. \\
& \left.-q_{12} q_{13}-x^{* *} q_{11} y^{* *} q_{14}-q_{15} q_{16}\right) v a_{2} x^{* * 2} \\
& -x^{* *} q_{11} q_{15} q_{16} q_{18}+\left(q_{12} q_{13}+x^{* *} q_{11} y^{* *} q_{14}\right) d_{2} z^{* *} q_{18} \\
& +\left(q_{12} q_{13}+x^{* *} q_{11} y^{* *} q_{14}+q_{15} q_{16}-x^{* *} q_{11} d_{2} z^{* *}\right. \\
& \left.-y^{* *} q_{14} d_{2} z^{* *}\right) q_{17} q_{18} \\
& Q_{5}=s_{1} x^{* *}\left(y^{* *} q_{14} d_{2} z^{* *}-q_{15} q_{16}\right)\left(a_{1} v C_{0}^{* *}+a_{2}^{2} C_{E}^{* *} x^{* *}\right) \\
& +\left(x^{* *} q_{11} q_{15} q_{16}-q_{12} q_{13} d_{2} z^{* *}-x^{* *} q_{11} y^{* *} q_{14} d_{2} z^{* *}\right) v x^{* *} a_{2} x^{* *} \\
& -x^{* *} q_{11} q_{15} q_{16} q_{17} q_{18}+\left(q_{12} q_{13}+x^{* *} q_{11} y^{* *} q_{14}\right) d_{2} z^{* *} q_{17} q_{18}
\end{aligned}
$$

and,

$$
q_{11}=\frac{q_{1} y^{* *}}{\left(1+x^{* *}\right)^{2}}-\frac{1}{k}, q_{12}=\frac{q_{1} x^{* *}}{1+x^{* *}}, q_{13}=\frac{q_{2} y^{* *}}{\left(1+x^{* *}\right)^{2}}, q_{14}=\frac{q_{3} z^{* *}}{\left(1+y^{* *}\right)^{2}}
$$

$-r_{1}, \quad q_{15}=\frac{q_{3} *^{* *}}{1+y^{* *}}, q_{16}=\frac{q_{4} z^{* *}}{\left(1+y^{* *}\right)^{2}}, q_{17}=a_{1}+a_{2} x^{* *}, \quad q_{18}=$ $b_{1}+v x^{* *}$. According to Routh-Hurwitz's criteria for the equilibrium point $E_{23}^{* *}$ to be locally asymptotically stable, following conditions must be satisfied

$Q_{i}>0, \quad i=1,2,3,4,5, \quad Q_{1} Q_{2}>Q_{3}$,

$Q_{1} Q_{2} Q_{3}>\left(Q_{3}^{2}+Q_{1}^{2} Q_{4}\right)$ and $\left(Q_{3} Q_{4}-Q_{2} Q_{5}\right) \quad\left(Q_{1} Q_{2}\right.$ $\left.-Q_{3}\right)>\left(Q_{1} Q_{4}-Q_{5}\right)^{2}$. It is difficult to interpret the results in ecological terms from these complicated expressions, however, numerical examples are taken and graphs are plotted to illustrate the dynamical behaviour of the system about equilibrium $E_{23}^{* *}$.

\section{Hopf-bifurcation}

Again, in the similar way the equilibrium population densities are functions of $s_{1}$ and the coefficients of the characteristic Eq. (90) are functions of the parameter $s_{1}$. Now we can use the notation $Q_{i}=Q_{i}\left(s_{1}\right)$ for $i=1,2,3,4,5$. Now noting that the quantities $Q_{i} s$ are smooth functions of the parameter $s_{1}$. As we have explained the definition of Hopf-bifurcation in previous section. Without knowing eigenvalues, Liu (1994) proved that (referring the result to the current case): if $Q_{i}\left(s_{1}\right)$,

$$
\begin{aligned}
& \Delta_{1}\left(s_{1}\right)=Q_{1}\left(s_{1}\right) Q_{2}\left(s_{1}\right)-Q_{3}\left(s_{1}\right), \\
& \Delta_{2}\left(s_{1}\right)=Q_{1}\left(s_{1}\right) Q_{2}\left(s_{1}\right) Q_{3}\left(s_{1}\right)-\left(Q_{3}^{2}\left(s_{1}\right)+Q_{1}^{2}\left(s_{1}\right) Q_{4}\right.
\end{aligned}
$$
$\left.\left(s_{1}\right)\right)$,

$$
\Delta_{3}\left(s_{1}\right)=\left[Q_{3}\left(s_{1}\right) Q_{4}\left(s_{1}\right)-Q_{2}\left(s_{1}\right) \quad Q_{5}\left(s_{1}\right)\right]\left[Q_{1}\left(s_{1}\right) Q_{2}\left(s_{1}\right)\right.
$$
$\left.-Q_{3}\left(s_{1}\right)\right]-\left[Q_{1}\left(s_{1}\right) Q_{4}\left(s_{1}\right)-Q_{5}\left(s_{1}\right)\right]^{2}$ are smooth functions of the parameter ' $s_{1}$ ' in an open interval containing $s_{1}^{* *} \epsilon \mathfrak{R}^{+}$such that following conditions hold: (iii ${ }_{*}$ ) $Q_{1}\left(s_{1}^{* *}\right)>0, \Delta_{1}\left(s_{1}^{* *}\right)>0, \Delta_{2}\left(s_{1}^{* *}\right)>0$ and $\Delta_{3}\left(s_{1}^{* *}\right)=0$; $\left(\mathrm{iv}_{*}\right) d \Delta_{3}\left(s_{1}\right) /\left.d s_{1}\right|_{s_{1}=s_{1}^{* *}} \neq 0$ then $\left(\mathrm{iii}_{*}\right)$ and $\left(\mathrm{iv}_{*}\right)$ are equivalent to conditions (i) and (ii) for the occurrence of a simple Hopf-bifurcation at $s_{1}=s_{1}^{* *}$. Hence, in the similar way, we can propose the following theorem:

Theorem 5 If a critical value $s_{1}^{* *}$ of parameter $s_{1}$ be found such that $Q_{i}\left(s_{1}^{* *}\right)>0, \Delta_{1}\left(s_{1}^{* *}\right)>0, \Delta_{2}\left(s_{1}^{* *}\right)>0, \Delta_{3}\left(s_{1}^{* *}\right)=$ 0 and further $\Delta_{3}^{\prime} \neq 0$ (where primes denotes differentiation with respect to $s_{1}$ ) then the system (7)-(11) undergoes Hopfbifurcation around $E_{23}^{* *}$. (for proof see Appendix 1).

\section{Global stability}

Theorem 6 If the following inequalities hold in the region $\Omega$

$$
\begin{aligned}
& k q_{1} y^{* *}<\pi_{21} \\
& v x^{* *}<a_{1}+a_{2} x^{* *} \\
& 2\left[H_{1} q_{3}\left(1+y^{* *}\right)+q_{4} H_{2}\right]^{2}<H_{1} H_{2} d_{2} \pi_{22}^{2}\left(r_{1}+\frac{q_{3} z^{* *}}{\pi_{22}}\right)
\end{aligned}
$$

$4 a_{2} x^{* *}>H_{3}\left(b_{1}+v x^{* *}\right)\left(a_{1}+\left(a_{2}-v\right) x^{* *}\right)$

where,

$$
\begin{aligned}
& H_{1}=\frac{q_{1}\left(1+x^{* *}\right)}{q_{2}}>0 \\
& H_{2}<\left(\frac{1}{k}-\frac{q_{1} y^{* *}}{\pi_{21}}\right) \frac{a_{1}+\left(a_{2}-v\right) x^{* *}}{6\left(a_{2} C_{E}-v C_{0}\right)^{2}} \\
& H_{3}=\frac{s_{1}}{a_{2} C_{E}-v C_{0}}>0
\end{aligned}
$$

where, $\pi_{21}=\left(1+s_{0} k\right)\left(1+x^{* *}\right), \pi_{22}=\left(1+K_{1}\right)\left(1+y^{* *}\right)$, then the positive equilibrium $E_{23}^{* *}$ is globally asymptotically 
stable with respect to all solutions initiating in the interior of positive region $\Omega$. (for proof see Appendix 2).

\section{Analysis of Model 3}

\section{Equilibria of Model 3}

The Model 3 has following four non-negative equilibria in $x, y, z$ space namely, $E_{30}=(0,0,0), \ddot{E_{31}}=(\ddot{x}, 0,0), \breve{E_{32}}=$ $(\breve{x}, \breve{y}, 0)$ and $\tilde{E_{33}}=(\tilde{x}, \tilde{y}, \tilde{z})$. The existence of $E_{30}$ is obvious. We prove the existence of $\ddot{E_{31}}, \widetilde{E_{32}}$ and $\tilde{E_{33}}$ as follows:

1. Existence of $\ddot{E_{31}}=(\ddot{x}, 0,0)$, where,

$$
\ddot{x}=s_{0} k
$$

2. Existence of $\breve{E_{32}}=(\breve{x}, \breve{y}, 0)$, where,

$$
\breve{y}=\left(s_{0}-\frac{\breve{x}}{k}\right)\left(\frac{1+\breve{x}}{q_{1}}\right)>0
$$

$\breve{y}>0$ if $s_{0} k>\breve{x}$. Using (99) in (13), we get

$$
C_{1} \breve{x}^{3}+C_{2} \breve{x}^{2}+C_{3} \breve{x}-C_{4}=0
$$

where, $C_{1}=r_{1}, C_{2}=r_{1}\left(2-s_{0} k\right)>0, C_{3}=q_{1} k\left(q_{2}-\right.$ $\left.r_{0}\right)-r_{1}\left(2 s_{0} r_{1}-1\right)>0$ and $C_{4}=k\left(s_{0} r_{1}+r_{0} q_{1}\right)$. The Eq. (100) will have a positive root $\breve{x}$ always.

3. Existence of $\tilde{E_{33}}=(\tilde{x}, \tilde{y}, \tilde{z})$

Here, $\tilde{x}, \tilde{y}$ and $\tilde{z}$ are the positive solutions of the system of algebraic equations:

from (14),

$z=\left(\frac{q_{4} y}{1+y}-d_{1}\right) \frac{1}{d_{2}}=S_{1}(y)$

Now, considering two functions [from (12) and (13)],

$$
\begin{aligned}
& K_{11}(x, y)=s_{0} k(1+x)-x(1+x)-q_{1} k y \\
& K_{12}(x, y)=s_{0}-\frac{x}{k}+\left(\frac{q_{2} x-q_{1} y}{1+x}\right)-\frac{q_{3} z}{1+y}-\left(r_{0}+r_{1} y\right)
\end{aligned}
$$

For existence of $\tilde{x}$ and $\tilde{y}$, the two isoclines,

$K_{11}(x, y)=0$

$K_{12}(x, y)=0$

must intersect. We note that

$$
\begin{aligned}
& K_{11}(0,0)=s_{0} k>0 \\
& K_{12}(0,0)=s_{0}-r_{0}>0 \\
& K_{12}(0,0)>0 \text { if } s_{0}>r_{0} .
\end{aligned}
$$

Also, $K_{11}(x, 0)=0$ then $x$ will have one positive root (say) $\psi_{1}$, from the following quadratic equation of $x$,

$x^{2}-x\left(s_{0} k-1\right)-s_{0} k=0$

if $s_{0} k>1$.

$$
K_{11}(0, y)=0 \text { then } y=\frac{s_{0}}{q_{1}}=\psi_{2} \text { (say). }
$$

$K_{12}(x, 0)=0$ then $x=$ will have one positive root (say) $\psi_{3}$, from the following quadratic equation of $x$,

$$
\begin{aligned}
& d_{2} x^{2}-x\left[k d_{1} q_{3}+k d_{2}\left(s_{0}-r_{0}\right)+d_{2}\left(k q_{2}-1\right)\right] \\
& \quad-k\left[d_{1} q_{3}+d_{2}\left(s_{0}-r_{0}\right)\right]=0
\end{aligned}
$$

if $s_{0}>r_{0}$ and $k q_{2}>1$.

$K_{12}(0, y)=0$ then $y$ will have one positive root (say) $\psi_{4}$, from the following cubic equation of $y$,

$N_{1} y^{3}+N_{2} y^{2}-N_{3} y-N_{4}=0$

$N_{1}=\left(r_{1}+q_{1}\right) d_{2}, \quad N_{2}=d_{2}\left[2\left(q_{1}+r_{1}\right)-\left(s_{0}-r_{0}\right)\right]>0$, $N_{3}=2 d_{2}\left(s_{0}-r_{0}\right)-\left[\left(r_{1}+q_{1}\right) d_{2}+q_{3}\left(q_{4}-d_{1}\right)\right]>0$ and $N_{4}=d_{1} q_{3}+\left(s_{0}-r_{0}\right) d_{2}>0$. Thus both the isoclines intersect each other in the region

$G_{11}=\left\{(x, y): 0<x<\psi_{3}, 0<y<\psi_{2}\right\}$

in the following two cases: (see Fig. 3)

Case $(i): \psi_{3}>\psi_{1}, \psi_{2}>\psi_{4}$

Case (ii) : $\psi_{3}<\psi_{1}, \psi_{2}<\psi_{4}$

This point of intersection will give $\tilde{x}, \tilde{y}$. For uniqueness of $(\tilde{x}, \tilde{y})$, we must have $\frac{d y}{d x}<0$ for both the curves in the region $G_{11}$.

For curve (104),

$\frac{d y}{d x}=\frac{s_{0} k-(2 x+1)}{q_{1} k}<0$

and for curve (105),

$\frac{d y}{d x}=\frac{\frac{1}{(1+x)^{2}}\left(q_{1} y+q_{2}\right)-\frac{1}{k}-\frac{S_{1}^{\prime}(y) q_{3}}{1+y}}{\left(r_{1}+\frac{q_{1}}{1+x}-\frac{S_{1}(y) q_{3}}{(1+y)^{2}}\right)}<0$

In case (i), the absolute value of $\frac{d y}{d x}$ given by (108) is less than the absolute value of $\frac{d y}{d x}$ given by (109). For the case (ii), just the opposite is the condition.

Knowing the value of $\tilde{x}$ and $\tilde{y} ; \tilde{z}$ can be computed from the Eq. (101).

\section{Dynamical behaviour of the Model 3}

Local stability

The general variational matrix corresponding to the Model 3 is 
Fig. 3 Existence of equilibrium point $\tilde{E_{33}}$ of Model 3
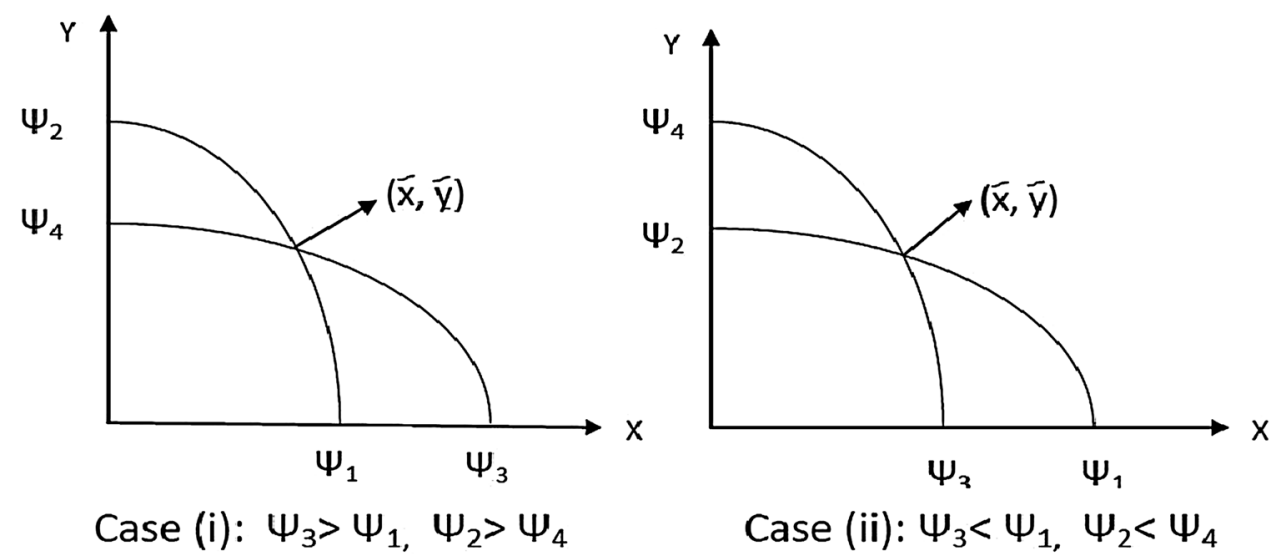

$J(x, y, z)=\left[\begin{array}{ccc}b_{11} & -b_{12} & 0 \\ b_{21} & b_{22} & -b_{23} \\ 0 & b_{32} & b_{33}\end{array}\right]$

where,

$b_{11}=s_{0}-\frac{2 x}{k}-\frac{q_{1} y}{(1+x)^{2}}, \quad b_{12}=\frac{q_{1} x}{(1+x)}, \quad b_{21}=\frac{q_{2} y}{(1+x)^{2}}, \quad b_{22}=$ $\frac{q_{2} x}{(1+x)}-\frac{q_{3} z}{(1+y)^{2}}-r_{0}-2 r_{1} y, \quad b_{23}=\frac{q_{3} y}{(1+y)}, \quad b_{32}=\frac{q_{4} z}{(1+y)^{2}}, \quad b_{33}=$ $\frac{q_{4} y}{1+y}-d_{1}-2 d_{2} z$.

1. About $E_{30}$, the eigenvalues of the characteristic equation are $s_{0},-r_{0}$ and $-d_{1}$, which shows that $E_{30}$ is unstable.

2. About $\ddot{E_{31}}$, the eigen values of the characteristic equation are $-s_{0}, \frac{q_{2} \ddot{x}}{1+\ddot{x}}-r_{0}$ and $-d_{1}$, which shows $\ddot{E_{31}}$ is locally asymptotically stable if

$q_{2} \ddot{x}<r_{0}(1+\ddot{x})$

which implies that,

$q_{2}-r_{0}<\frac{r_{0}}{s_{0} k}$

holds good.

Remark 4 From (111), it may be noted that if the difference of the growth rate of intermediate predator and its natural death rate is less than its natural death rate is divided by the product of intrinsic growth rate and carrying capacity of the prey then the prey population will only survive and both the predators will tend to extinction.

3. About $\widetilde{E_{32}}$, the eigen values of the characteristic equation are $\frac{q_{1} \breve{y}}{(1+\breve{x})^{2}}-\frac{1}{k},-r_{1} \breve{y}$ and $\frac{q_{4} \breve{y}}{(1+\breve{y})}-d_{1}$, which shows $\breve{E_{32}}$ is locally asymptotically stable if $q_{1} \breve{y} k<(1+\breve{x})^{2}$

which implies that,

$\breve{x}<s_{0} k<(1+2 \breve{x})$ and

$0<\breve{y}<\frac{d_{1}}{q_{4}-d_{1}}$

if $d_{1}<q_{4}$, holds good.

Remark 5 From (113) and (114), following may be noted:

(i) if the equilibria of prey is less than the product of intrinsic growth rate and carrying capacity of prey, which is again less than the sum of unity and twice of equilibria of prey,

(ii) if the equilibria of intermediate predator is less than the ratio of natural death rate of top predator and the natural death rate of top predator is subtracted from the growth rate of top predator, then prey and intermediate predator population will survive and top predator population will tend to extinction.

4. About $\tilde{E_{33}}$, the characteristic equation is given by

$\lambda^{3}+B_{1} \lambda^{2}+B_{2} \lambda+B_{3}=0$

where,

$B_{1}=d_{2} \tilde{z}+\tilde{x}\left(\frac{1}{k}-\frac{q_{1} \tilde{y}}{(1+\tilde{x})^{2}}\right) \quad+\tilde{y}\left(r_{1}-\frac{q_{3} \tilde{z}}{(1+\tilde{y})^{2}}\right), \quad B_{2}=\frac{q_{1} q_{2} \tilde{x} \tilde{y}}{(1+\tilde{x})^{3}}$ $+\frac{q_{3} q_{4} \tilde{y} \tilde{z}}{(1+\tilde{y})^{3}}+\tilde{y} \tilde{z} d_{2}\left(r_{1}-\frac{q_{3} \tilde{z}}{(1+\tilde{y})^{2}}\right)+\tilde{x}\left(\frac{1}{k}-\frac{q_{1} \tilde{y}}{(1+\tilde{x})^{2}}\right)\left[\tilde{z} d_{2}+\tilde{y}\left(r_{1}-\right.\right.$ $\left.\left.\frac{q_{3} \tilde{z}}{(1+\tilde{y})^{2}}\right)\right], \quad B_{3}=\frac{\tilde{x} \tilde{z} \tilde{q} q_{1} q_{2} d_{2}}{(1+\tilde{x})^{3}}+\tilde{x} \tilde{y} \tilde{z}\left(\frac{1}{k}-\frac{q_{1} \tilde{y}}{(1+\tilde{x})^{2}}\right)\left[d_{2}\left(r_{1}-\frac{q_{3} \tilde{z}}{(1+\tilde{y})^{2}}\right)+\right.$ $\left.\frac{q_{3} q_{4}}{(1+\tilde{y})^{3}}\right]$.

According to Routh-Hurwitz criteria $\tilde{E_{33}}$ is locally asymptotically stable if $B_{1}>0, B_{2}>0, B_{3}>0$ and $B_{1} B_{2}>B_{3}$, i.e.,

$\theta_{3}\left(\tilde{x} \theta_{1}+\tilde{y} \theta_{2}\right)+\left(\tilde{y} \theta_{2}+\tilde{z} d_{2}\right)\left[\tilde{x} \theta_{1}\left(\tilde{x} \theta_{1}+\tilde{y} \theta_{2}+d_{2} \tilde{z}\right)+\left(\theta_{4}\right.\right.$

$\left.\left.+\tilde{y} \tilde{z} d_{2} \theta_{2}\right)\right]>0$, and

$q_{1} \tilde{y} k<(1+\tilde{x})^{2}$,

$q_{3} \tilde{z}<r_{1}(1+\tilde{y})^{2}$. 
Where, $\quad \theta_{1}=\frac{1}{k}-\frac{q_{1} \tilde{y}}{(1+\tilde{x})^{2}}>0, \quad \theta_{2}=r_{1}-\frac{q_{3} \tilde{z}}{(1+\tilde{y})^{2}}>0, \quad \theta_{3}=$ $\frac{\tilde{x} \tilde{y} q_{1} q_{2}}{(1+\tilde{x})^{3}}$ and $\theta_{4}=\frac{\tilde{y} z q_{3} q_{4}}{(1+\tilde{y})^{3}}$.

Remark 6 Following may be noted from the above conditions (116-117):

(i) if the product of predation rate of prey and the equilibria of intermediate predator and the carrying capacity of prey is less than the square of the sum of unity and the equilibria of prey,

(ii) if the product of predation rate of intermediate predator and the equilibria of top predator less than the product of intraspecies competition coefficient among intermediate predator and the square of the sum of unity and the equilibria of intermediate predator then all the populations will survive.

\section{Hopf-bifurcation}

We are now in a position to make an attempt to find out the conditions under which the system undergoes Hopf-bifurcation. For this purpose, we choose the parameter $q_{4}$ as bifurcation parameter as it plays a crucial role in Holling type II functional response which describes the predation of intermediate consumer. We shall now apply the Liu's criteria, Liu (1994) to obtain the conditions for small amplitude periodic solution arising from Hopf-bifurcation.

As the equilibrium population densities are functions of $q_{4}$, the coefficients of the characteristic Eq. (115) are functions of the parameter $q_{4}$ and hence we can use the notation $B_{i}=B_{i}\left(q_{4}\right)$ for $i=1,2,3$. Noting that the quantities $B_{i} s$ are smooth functions of the parameter $q_{4}$, we first state in our case, the definition of a simple Hopf-Bifurcation.

If a critical value $\tilde{q_{4}}$ of parameter $q_{4}$ be found such that (i) a simple pair of complex conjugate eigenvalues of characteristic equation exists, say, $\lambda_{1}\left(q_{4}\right)=u\left(q_{4}\right)+i v\left(q_{4}\right)$, $\lambda_{2}\left(q_{4}\right)=u\left(q_{4}\right)-i v\left(q_{4}\right)=\overline{\lambda_{4}}\left(q_{4}\right)$. These eigen values will become purely imaginary at $q_{4}=\tilde{q_{4}}$, i.e. $\lambda_{1}\left(\tilde{q_{4}}\right)=i v_{0}$, $\lambda_{2}\left(\tilde{q_{4}}\right)=-i v_{0}$, with $v\left(\tilde{q_{4}}\right)=v_{0}>0$, and the other eigenvalue remains real and negative; and (ii) the transversality condition, $d \operatorname{Re} \lambda_{i}\left(\tilde{q_{4}}\right) /\left.d q_{4}\right|_{q_{4}=\tilde{q_{4}}}=d u\left(q_{4}\right) /\left.d q_{4}\right|_{q_{4}=\tilde{q_{4}}} \neq 0$ is satisfied. Then we find at $q_{4}=\tilde{q_{4}}$, a simple Hopf-bifurcation. Without knowing eigenvalues, Liu (1994) proved that (referring the result to the current case): if $B_{1}\left(q_{4}\right), B_{3}\left(q_{4}\right), \Delta\left(q_{4}\right)=B_{1}\left(q_{4}\right) B_{2}\left(q_{4}\right)-B_{3}\left(q_{4}\right)$ are smooth functions of the parameter ' $q_{4}$ ' in an open interval containing $\tilde{q_{4}} \epsilon \mathfrak{R}^{+}$such that following conditions hold:

(i $\left.\mathrm{i}_{*}\right) B_{1}\left(\tilde{q_{4}}\right)>0, \Delta\left(\tilde{q_{4}}\right)=0, B_{3}\left(\tilde{q_{4}}\right)>0$; (ii $\left.{ }_{*}\right) d \Delta\left(q_{4}\right) /$ $\left.d q_{4}\right|_{q_{4}=\tilde{q_{4}}} \neq 0$

then $\left(i_{*}\right)$ and $\left(\mathrm{ii}_{*}\right)$ are equivalent to conditions (i) and (ii) for the occurrence of a simple Hopf-bifurcation at $q_{4}=\tilde{q_{4}}$. Hence we can propose the following theorem:
Theorem 7 If a critical value $\tilde{q_{4}}$ of parameter $q_{4}$ be found such that $B_{1}\left(\tilde{q_{4}}\right)>0, B_{3}\left(\tilde{q_{4}}\right)>0$ and $\Delta\left(\tilde{q_{4}}\right)=0$ and further $\Delta^{\prime} \neq 0$ (where prime denotes differentiation with respect to $q_{4}$ ) then system (12)-(14) undergoes Hopf-bifurcation around $\tilde{E_{33}}$. (for proof see Appendix 3 ).

\section{Global stability}

Theorem 8 If the following inequalities hold $k q_{1} \tilde{y}<(1+$ $\tilde{x})$ and $q_{3} \tilde{z}<r_{1}(1+\tilde{y})$ then the positive equilibrium $\tilde{E_{33}}$ is globally asymptotically stable with respect to all solutions initiating in the interior of positive region $\Omega$. (for proof see Appendix 4).

\section{Numerical simulation}

In this section, we demonstrate the dynamical behaviour of the distributed delay on the effect of toxicant in a three species food-chain system for the models with the help of numerical simulations to facilitate the interpretation of our mathematical findings. The figures illustrate the stability behaviour of all the equilibrium points of the models and bifurcation for non-trivial equilibrium points for the given sets of parameters and the graphs have been plotted with the help of MATLAB.

\section{Numerical simulation for Model 3}

We choose the following values of parameters for $\ddot{E_{31}}$ :

$$
\begin{aligned}
& s_{0}=0.9945 ; \quad k=25 ; \quad q_{1}=0.47 ; \\
& \quad q_{2}=0.334 ; \quad q_{3}=0.381 ; \\
& q_{4}=0.12 ; \quad r_{0}=1.02423 ; \quad r_{1}=0.0257 ; \\
& \quad d_{1}=1.0571 ; \quad d_{2}=0.02483 .
\end{aligned}
$$

It is found that under the above set of parameters, the equilibrium point $\ddot{E_{31}}$ is

$\ddot{x}=24.8623, \quad \ddot{y}=0.0000$,

is locally asymptotically stable (see Fig. 4).

We choose the following values of parameters for $\breve{E_{32}}$ :

$$
s_{0}=1.07989 ; \quad r_{0}=0.002423 q_{3}=0.681 ;
$$$$
q_{4}=0.672 ; \quad d_{1}=0.671 ;
$$

With the above values of parameters and taking the remaining parameters to be the same as considered for $\ddot{E_{31}}$, it is found that under the above set of parameters, the equilibrium point $\breve{E_{32}}$ is

$\breve{x}=20.1283, \quad \breve{y}=12.2867, \quad \breve{z}=0.0000$

is locally asymptotically stable (see Fig. 5). 
Fig. 4 Time graph for the Model 3 (without toxicant and without distributed delay) around the equilibrium point $\ddot{E_{31}}$, showing the stability behavior

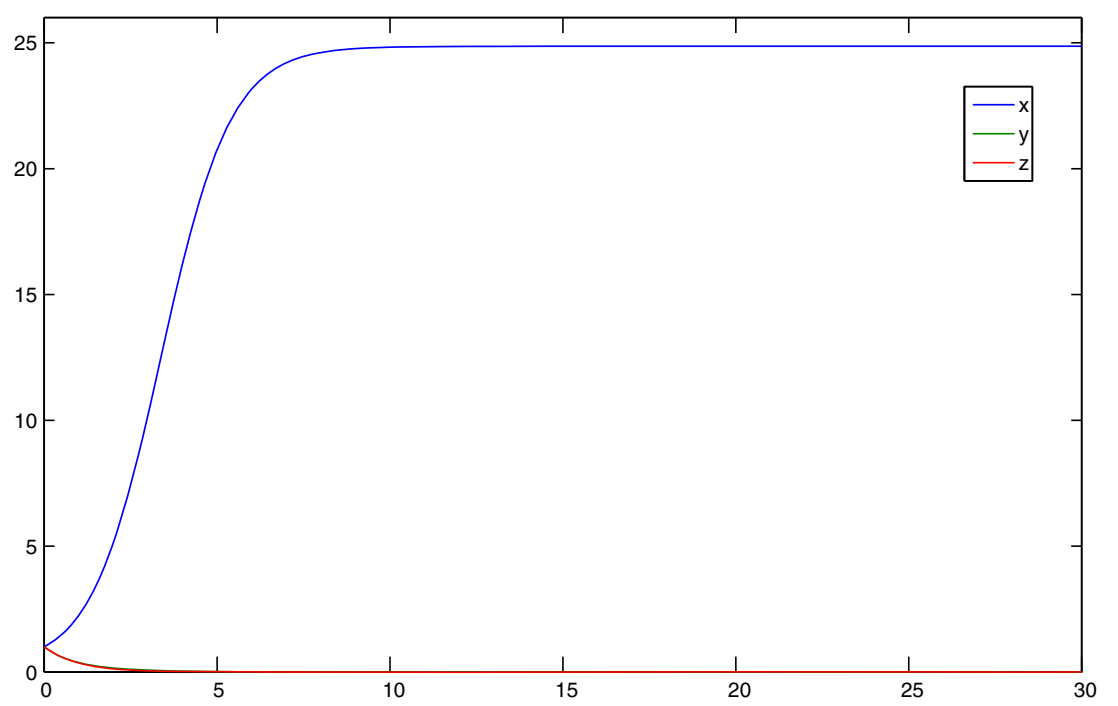

Fig. 5 Time graph for the Model 3 (without toxicant and without distributed delay) around the equilibrium point $\widetilde{E_{32}}$, showing the stability behavior

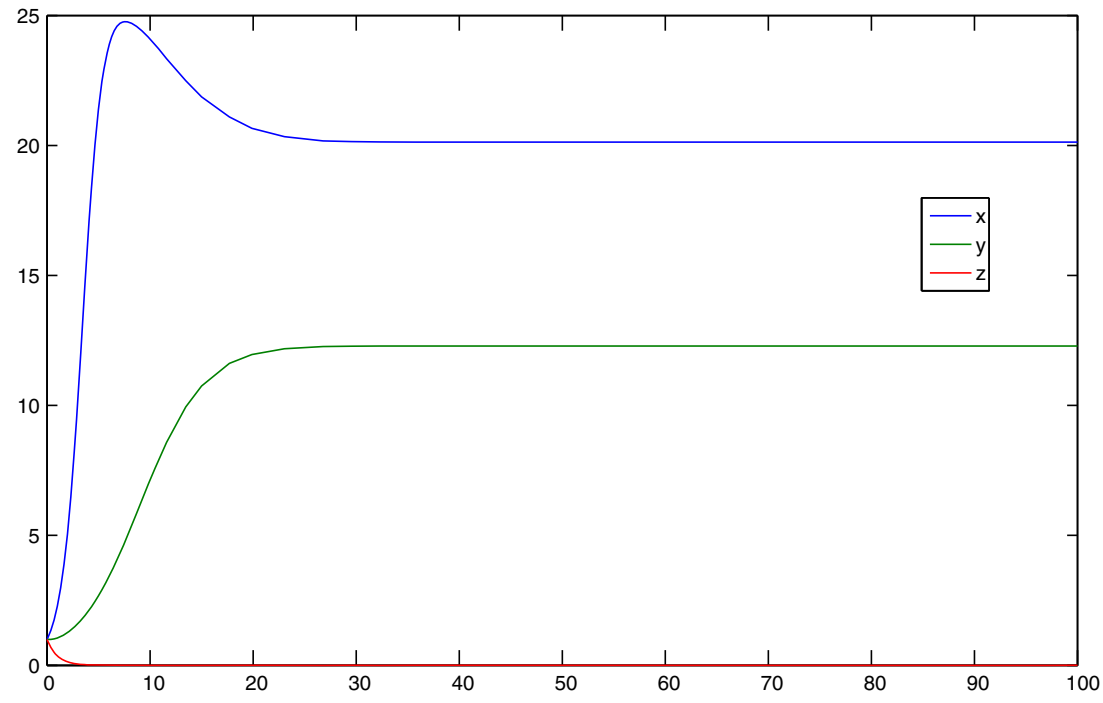

We choose the following values of parameters for $\tilde{E_{33}}$ :

$s_{0}=0.7845 ; \quad r_{0}=0.02423 ; \quad d_{1}=0.0571$.

With the above values of parameters and taking the remaining parameters to be the same as considered for $\ddot{E_{31}}$, it is found that under the above set of parameters, the equilibrium point $\tilde{E_{33}}$

$$
\tilde{x}=13.1430, \quad \tilde{y}=7.7885, \quad \tilde{z}=1.9833
$$

is locally asymptotically stable (see Fig. 6).

Now, we study the Hopf-bifurcation of the Model 3, taking $q_{4}$ as the bifurcating parameter. The transversality condition holds with the above set of parameters when $q_{4}=\tilde{q_{4}}=0.15$. It is clear that the interior equilibrium point $\tilde{E_{33}}$ of Model 3 is stable when $q_{4}>\tilde{q_{4}}$ and unstable when $q_{4} \leq \tilde{q_{4}}$ for which Hopf-bifurcation occurs (see Fig. 7).
Numerical simulation for Model 2

We choose the following values of parameters for $\hat{E_{21}}$ :

$$
\begin{aligned}
& s_{0}=0.7845 ; \quad k=25 ; \quad q_{1}=0.47 ; \quad q_{2}=0.334 ; \\
& \quad q_{3}=0.381 ; \quad q_{4}=0.12 ; \\
& r_{0}=1.02423 ; \quad r_{1}=0.0257 ; \quad d_{1}=1.0571 ; \\
& \quad d_{2}=0.02483 ; \quad s_{1}=0.01 ; \quad q_{0}=.515 ; \\
& a_{1}=0.71 ; \quad a_{2}=0.92 ; \quad v=0.102 ; \quad b_{1}=0.52 .
\end{aligned}
$$

It is found that under the above set of parameters, the equilibrium point

$$
\begin{gathered}
\hat{\hat{x}}=19.4046, \quad \hat{\hat{y}}=0.0000, \quad \hat{\hat{z}}=0.0000, \\
\hat{C_{E}}=0.1164, \quad \hat{C_{0}}=0.8314
\end{gathered}
$$

is locally asymptotically stable (see Fig. 8). 
Fig. 6 Time graph for the Model 3 (without toxicant and without distributed delay) around the equilibrium point $\tilde{E_{33}}$, showing the stability behavior
Fig. 7 Time graph for the Model 3 (without toxicant and without distributed delay) around the equilibrium point $\tilde{E_{33}}$, showing the bifurcation behavior at $q_{4}=0.15$
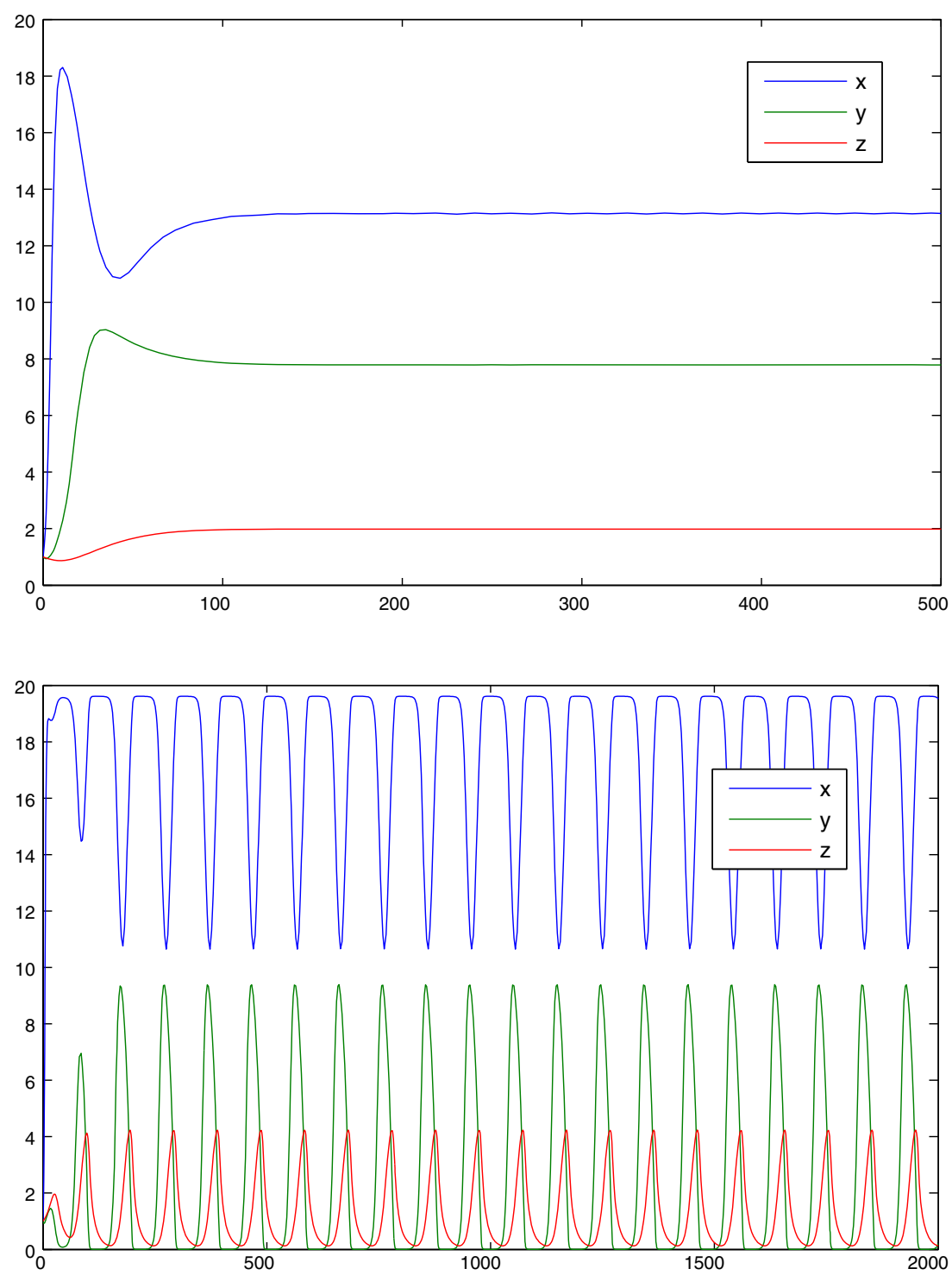

We choose the following values of parameters for $\overline{E_{22}}$ : $s_{0}=1.07845 ; \quad r_{0}=0.002423$.

With the above values of parameters and taking the remaining parameters to be the same as considered for $\hat{E_{21}}$, it is found that under the above set of parameters, the equilibrium point $\overline{E_{22}}$ is

$$
\begin{gathered}
\overline{\bar{x}}=17.0143, \quad \overline{\bar{y}}=11.3319, \quad \overline{\bar{z}}=0.0000, \\
\overline{\overline{C_{E}}}=0.1192, \quad \overline{\overline{C_{0}}}=0.8276
\end{gathered}
$$

is locally asymptotically stable (see Fig. 9).

We choose the following values of parameters for $E_{23}^{* *}$ : $r_{0}=0.02423 ; \quad d_{1}=0.0571$.

With the above values of parameters and taking the remaining parameters to be the same as considered for $\hat{E_{21}}$, it is found that under the above set of parameters, the equilibrium point $E_{23}^{* *}$ is

$$
\begin{gathered}
x^{* *}=12.8051, \quad y^{* *}=6.7579, \quad z^{* *}=1.9814, \\
C_{E}^{* *}=0.1267, \quad C_{0}^{* *}=0.8174
\end{gathered}
$$

is locally asymptotically stable (see Fig. 10).

Now, we study the Hopf-bifurcation of the Model 2, taking $s_{1}$ as the bifurcating parameter. The transversality condition holds with the above set of parameters when $s_{1}=s_{1}^{* *}=0.06$. It is clear that the interior equilibrium point $E_{23}^{* *}$ of Model 2 is stable when $s_{1}>s_{1}^{* *}$ and unstable when $s_{1} \leq s_{1}^{* *}$ for which Hopf-bifurcation occurs (see Fig. 11).

\section{Numerical simulation for Model 1}

We choose the following values of parameters for $\hat{E_{11}}$ : 
Fig. 8 Time graph for the Model 2 (with toxicant and without distributed delay) around the equilibrium point $\hat{E_{21}}$, showing the stability behavior
Fig. 9 Time graph for the Model 2 (with toxicant and without distributed delay) around the equilibrium point $\overline{E_{22}}$, showing the stability behavior
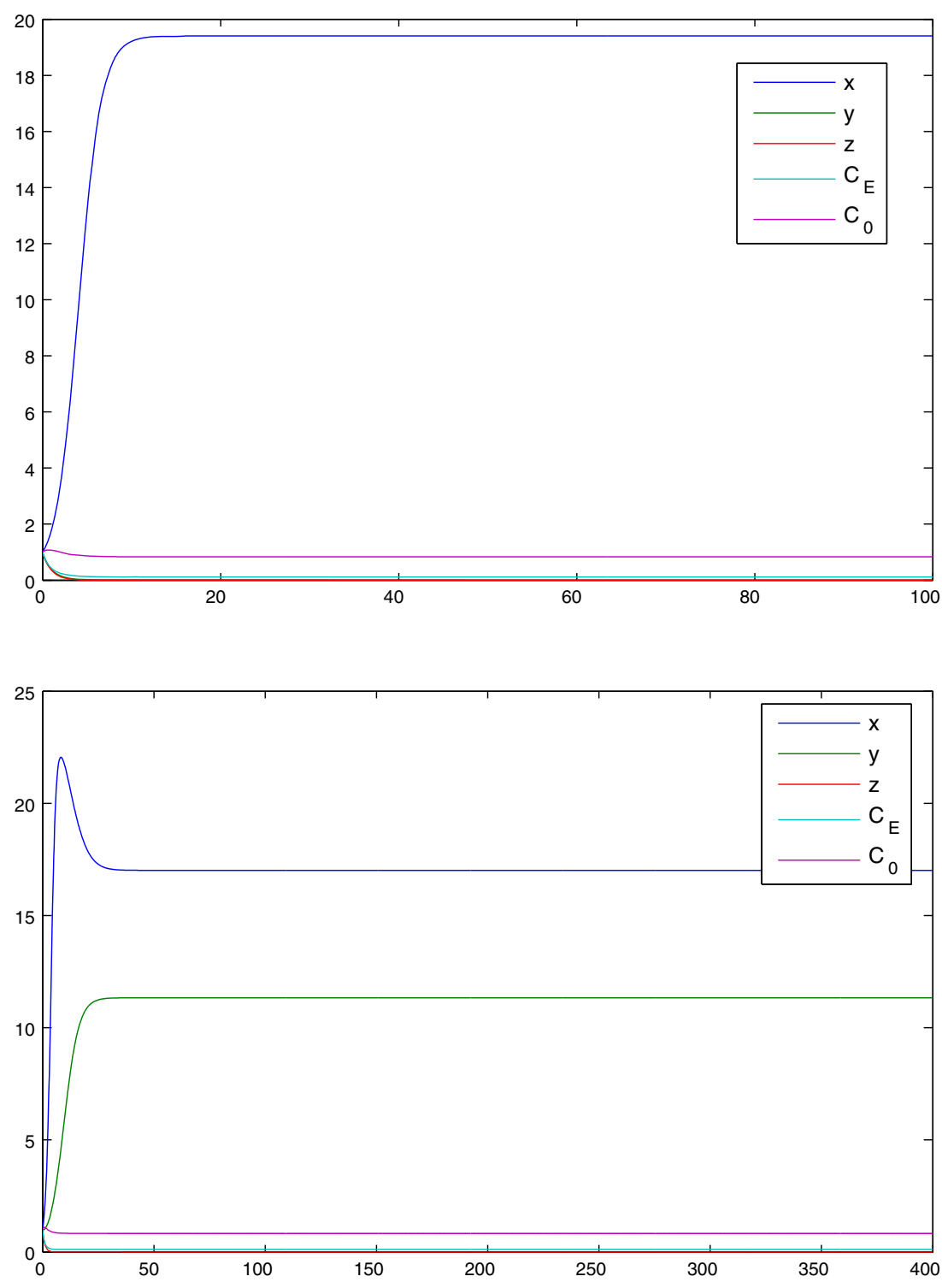

$$
\begin{aligned}
& s_{0}=0.7845 ; \quad k=25 ; \quad q_{1}=0.47 ; \quad q_{2}=0.334 ; \\
& \quad q_{3}=0.381 ; \quad q_{4}=0.12 ; \\
& r_{0}=1.02423 ; \quad r_{1}=0.0257 ; \quad d_{1}=1.0571 \\
& \quad d_{2}=0.02483 ; \quad s_{1}=0.05 ; \quad q_{0}=0.515 \\
& a_{1}=0.71 ; \quad a_{2}=0.0042 ; \quad v=0.102 ; \quad b_{1}=0.52 \\
& \quad \gamma_{1}=0.59
\end{aligned}
$$

It is found that under the above set of parameters, the equilibrium point $\hat{E_{11}}$

$$
\begin{gathered}
\hat{x}=18.5747, \quad \hat{y}=0.0000, \quad \hat{z}=0.0000, \\
\hat{C_{0}}=0.8302, \quad \hat{R_{1}}=0.1173
\end{gathered}
$$

is locally asymptotically stable (see Fig. 12).

We choose the following values of parameters for $\overline{E_{12}}$ :

$$
\begin{gathered}
s_{0}=0.9845 ; \quad r_{0}=0.002423 ; \quad a_{1}=0.61 ; \\
\gamma_{1}=0.59 ; \quad a_{2}=0.92 .
\end{gathered}
$$

With the above values of parameters and taking the remaining parameters to be the same as considered for $\hat{E_{11}}$, it is found that under the above set of parameters, the equilibrium point $\overline{E_{12}}$

$$
\begin{gathered}
\bar{x}=14.3096, \quad \bar{y}=12.0529, \quad \bar{z}=0.0000, \\
\overline{C_{0}}=0.8419, \quad \overline{R_{1}}=0.1268
\end{gathered}
$$

is locally asymptotically stable (see Fig. 13).

We choose the following values of parameters for $E_{13}^{*}$ :

$$
\begin{aligned}
& r_{0}=0.02423 ; \quad d_{1}=0.0571 ; \quad s_{1}=0.195 ; \\
& \quad q_{0}=0.0989 ; \quad a_{1}=0.89 ; \\
& a_{2}=0.23 ; \quad v=0.103 ; \quad b_{1}=0.07 ; \quad \gamma_{1}=1.32 ;
\end{aligned}
$$


Fig. 10 Time graph for the Model 2 (with toxicant and without distributed delay) around the equilibrium point $E_{23}^{* *}$, showing the stability behavior
Fig. 11 Time graph for the Model 2 (with toxicant and without distributed delay) around the equilibrium point $E_{23}^{* *}$, showing the bifurcation behavior at $s_{1}=0.06$
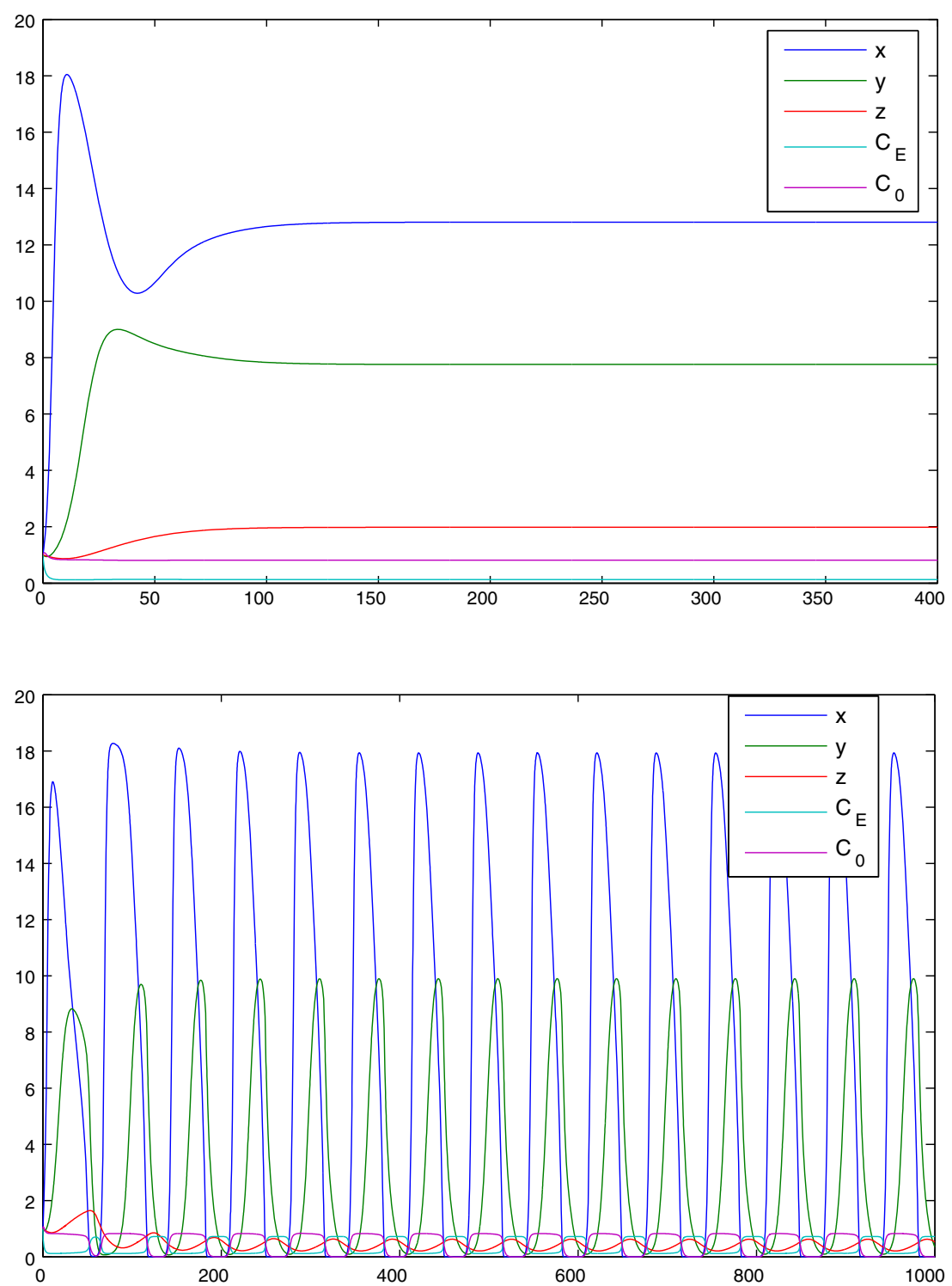

With the above values of parameters and taking the remaining parameters to be the same as considered for $\hat{E_{11}}$, it is found that under the above set of parameters, the equilibrium point $E_{13}^{*}$

$$
\begin{gathered}
x^{*}=11.7650, y^{*}=6.0464, z^{*}=1.4017, C_{E}^{*}=0.4176, \\
C_{0}^{*}=0.8819, R_{1}^{*}=0.4179
\end{gathered}
$$

is locally asymptotically stable (see Fig. 14).

These calculations prove that the equilibrium $E_{13}^{*}$ is stable when $\gamma_{1}>\gamma_{1}^{*}$ as is illustrated by the computer simulations (Fig. 14 and $\gamma_{1}=1.92$ ). When $\gamma_{1}$ passes through the critical value $\gamma_{1}^{*}=1.22$, i.e., when $\gamma_{1}<\gamma_{1}^{*}, E_{13}^{*}$ loses its stability and a Hopf bifurcation occurs (see Fig. 15); i.e., a family of periodic solutions bifurcate from the positive equilibrium $E_{13}^{*}$.

\section{Conclusion}

In this paper we have proposed and analyzed a nonlinear mathematical model to study the role of distributed delay in toxicant uptake process by exposed prey population in a three-species food-chain system under toxicant stress. It is assumed in the model that the growth rate of prey population is adversely affected by the toxicant concentration present in the prey population, which is indirectly effecting the predator populations.

It is concluded from the stability of $\ddot{E_{31}}$ of Model 3 that only the prey population would survive and both the predator populations would tend to extinction (see Fig. 4). From the stability of $\breve{E_{32}}$ of Model 3 it may be concluded that the prey and intermediate predator populations would 
Fig. 12 Time graph for the Model 1 (toxicant and distributed delay) around the equilibrium point $\hat{E_{11}}$, showing the stability behavior

Fig. 13 Time graph for the Model 1 (toxicant and distributed delay) around the equilibrium point $\overline{E_{12}}$, showing the stability behavior

Fig. 14 Time graph for the Model 1 (toxicant and distributed delay) around the equilibrium point $E_{13}{ }^{*}$, showing the stability behavior
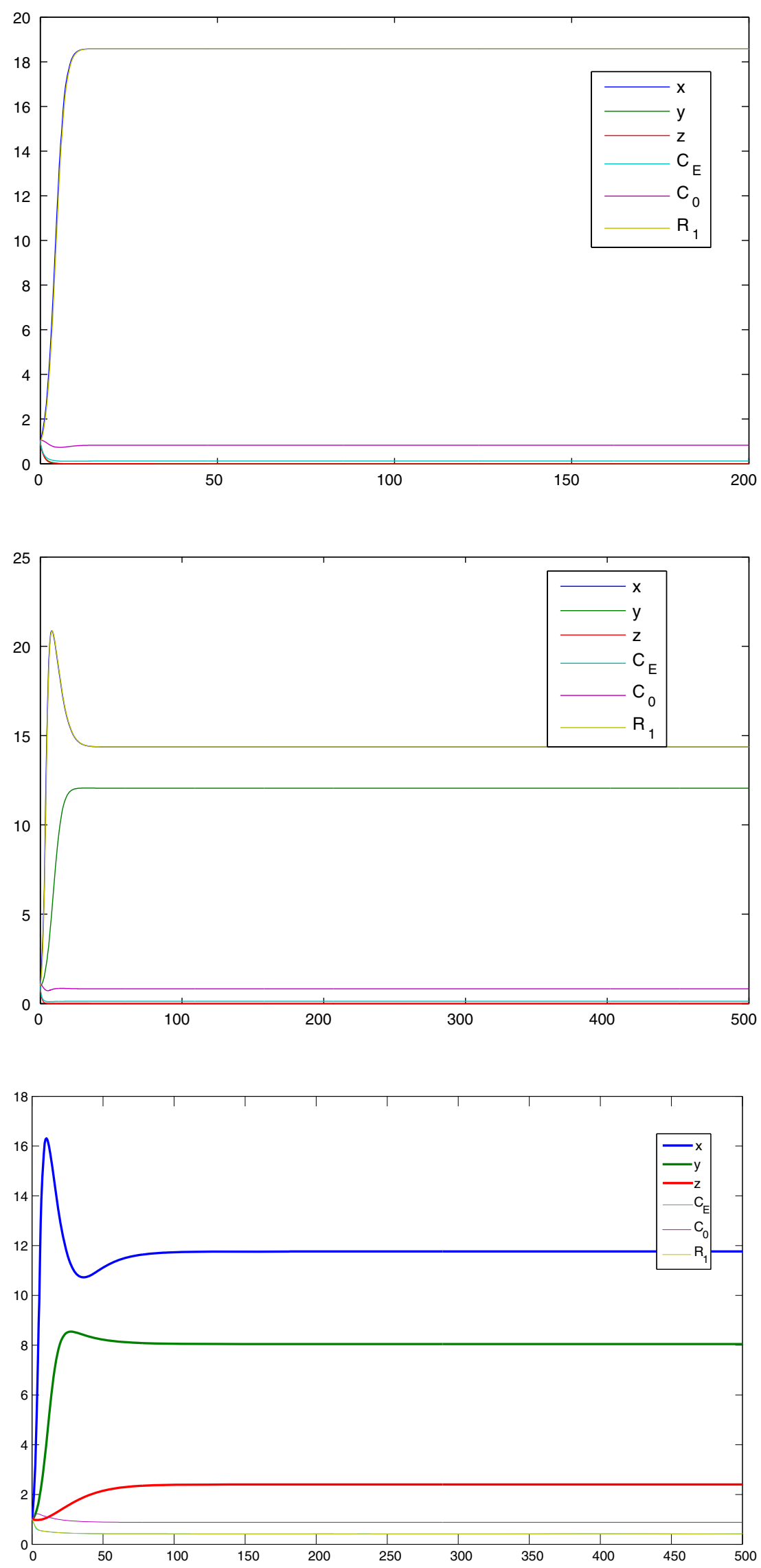
Fig. 15 Time graph for the Model 1 (toxicant and distributed delay) around the equilibrium point $E_{13}^{*}$, showing the bifurcation behavior at $\gamma_{1}=1.22$

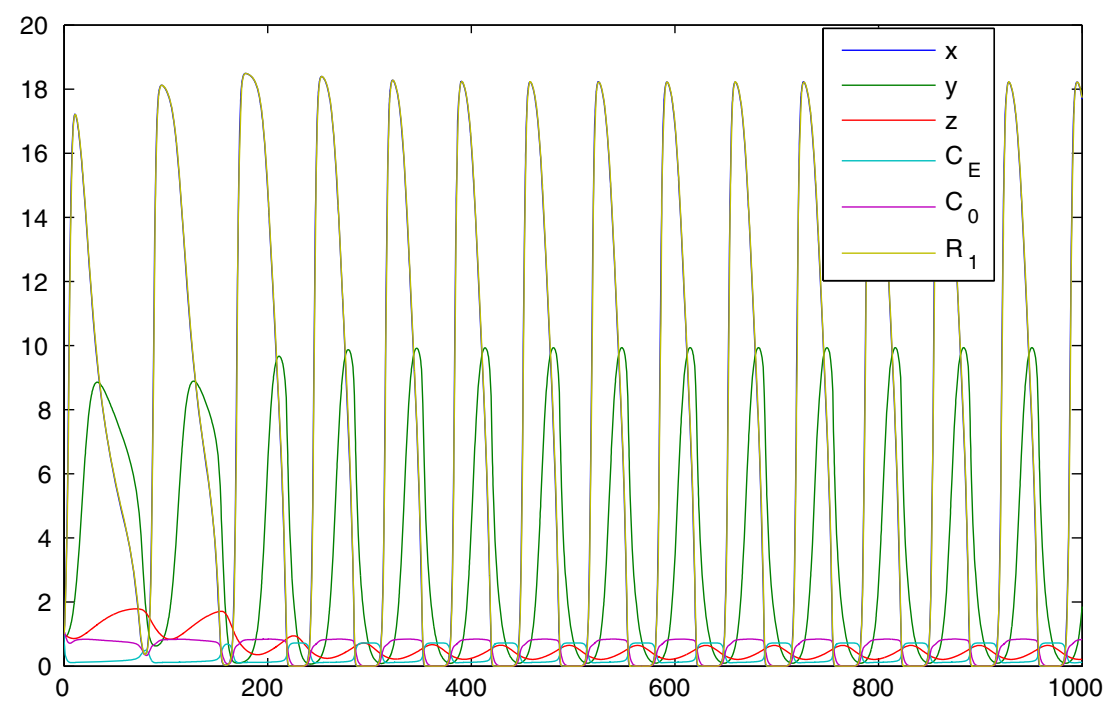

Fig. 16 Time series graph of Prey population for Model 3, Model 2 and Model 1 around the equilibrium points $\tilde{E_{33}}=(\tilde{x}, \tilde{y}, \tilde{z}), E_{23}^{* *}=$ $\left(x^{* *}, y^{* *}, z^{* *}, C_{E}^{* *}, C_{0}^{* *}\right)$ and $E_{13}^{*}=\left(x^{*}, y^{*}, z^{*}, C_{E}^{*}, C_{0}^{*}, R_{1}^{*}\right)$

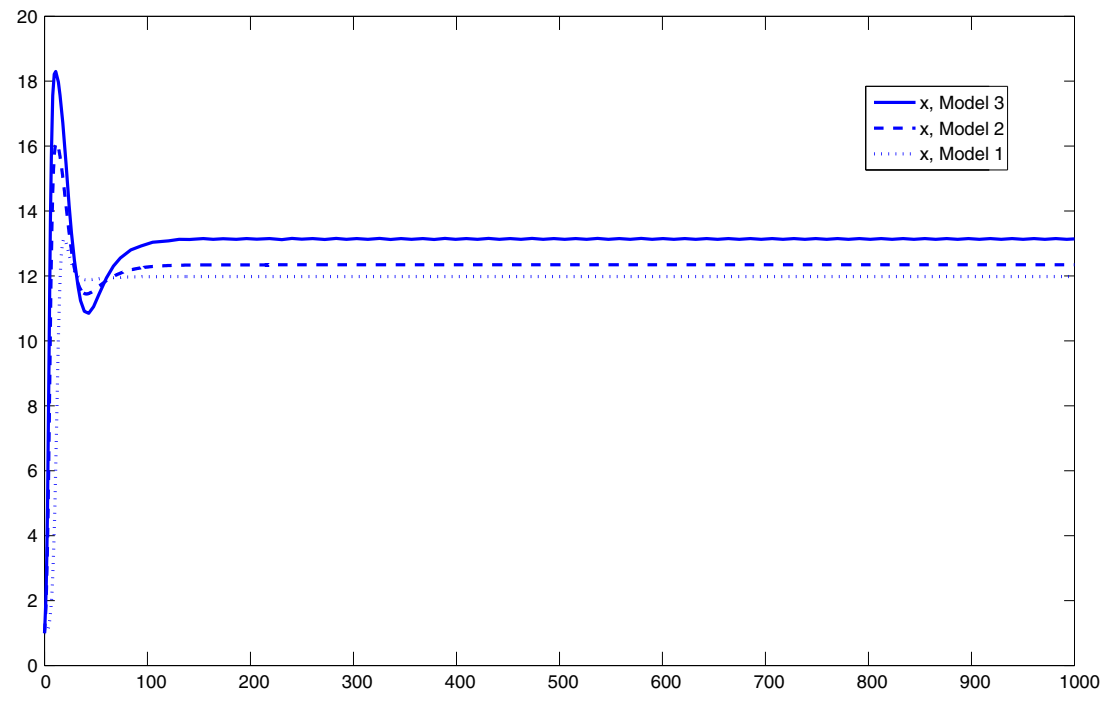

survive and the top predator population would tend to extinction (see Fig. 5). The stability of $\tilde{E_{33}}$ of Model 3 ensures the coexistence of all the three populations (see Fig. 6). From the stability of $\hat{E_{21}}$ of Model 2, it is concluded that only prey population will survive in the presence of toxicant, but at reduced equilibrium. It may be noted that due to decrease in prey population, both the predator populations may get extinct (see Remark 3 and Fig. 8). From the stability of $\overline{E_{22}}$ of Model 2 it may be concluded that the prey and intermediate predator population both will survive in the presence of toxicant, but their equilibrium levels decrease due to toxicant (see Fig. 9). Due to the decrease in intermediate predator population, the top predator population also tend to extinction. The stability of $E_{23}^{* *}$ of Model 2 ensures the coexistence of all the three populations but their equilibrium levels decrease due to the presence of toxicant (see Fig. 10). All the equilibrium points of Model 1 show the similar stability behavior as it is being shown in Model 2 but the equilibrium levels of each population in respective equilibrium points of Model 1 decreases further on account of distributed delay considered in toxicant uptake process by prey population (see Figs. 12, 13, 14, $16,17,18,19)$. After introducing the distributed delay in Model 2 the Hopf-bifurcation phenomenon is observed in Model 1 with respect to the delay parameter $\gamma_{1}$ and it is shown that when $\gamma_{1}$ is greater than its critical value, the stability behavior is observed and when $\gamma_{1}$ is less than its critical value then Hopf-bifurcation occurs and periodic solution is obtained (see Fig. 15). This phenomenon has been observed by many authors (Ruan and Wolkowicz (1996); Tabares et al. 2011). 
Fig. 17 Time series graph of intermediate predator population for Model 3, Model 2 and Model 1 around the equilibrium points

$\tilde{E_{33}}=(\tilde{x}, \tilde{y}, \tilde{z}), E_{23}^{* *}=$ $\left(x^{* *}, y^{* *}, z^{* *}, C_{E}^{* *}, C_{0}^{* *}\right)$ and $E_{13}^{*}=\left(x^{*}, y^{*}, z^{*}, C_{E}^{*}, C_{0}^{*}, R_{1}^{*}\right)$

Fig. 18 Time series graph of top predator population for Model 3, Model 2 and Model 1 around the equilibrium points $\tilde{E_{33}}=(\tilde{x}, \tilde{y}, \tilde{z}), E_{23}^{* *}=$ $\left(x^{* *}, y^{* *}, z^{* *}, C_{E}^{* *}, C_{0}^{* *}\right)$ and $E_{13}^{*}=\left(x^{*}, y^{*}, z^{*}, C_{E}^{*}, C_{0}^{*}, R_{1}^{*}\right)$

Fig. 19 Time graph for Model 3, Model 2 and Model 1 around the equilibrium points $\tilde{E_{33}}=(\tilde{x}, \tilde{y}, \tilde{z}), E_{23}^{* *}=$ $\left(x^{* *}, y^{* *}, z^{* *}, C_{E}^{* *}, C_{0}^{* *}\right)$ and $E_{13}^{*}=\left(x^{*}, y^{*}, z^{*}, C_{E}^{*}, C_{0}^{*}, R_{1}^{*}\right)$
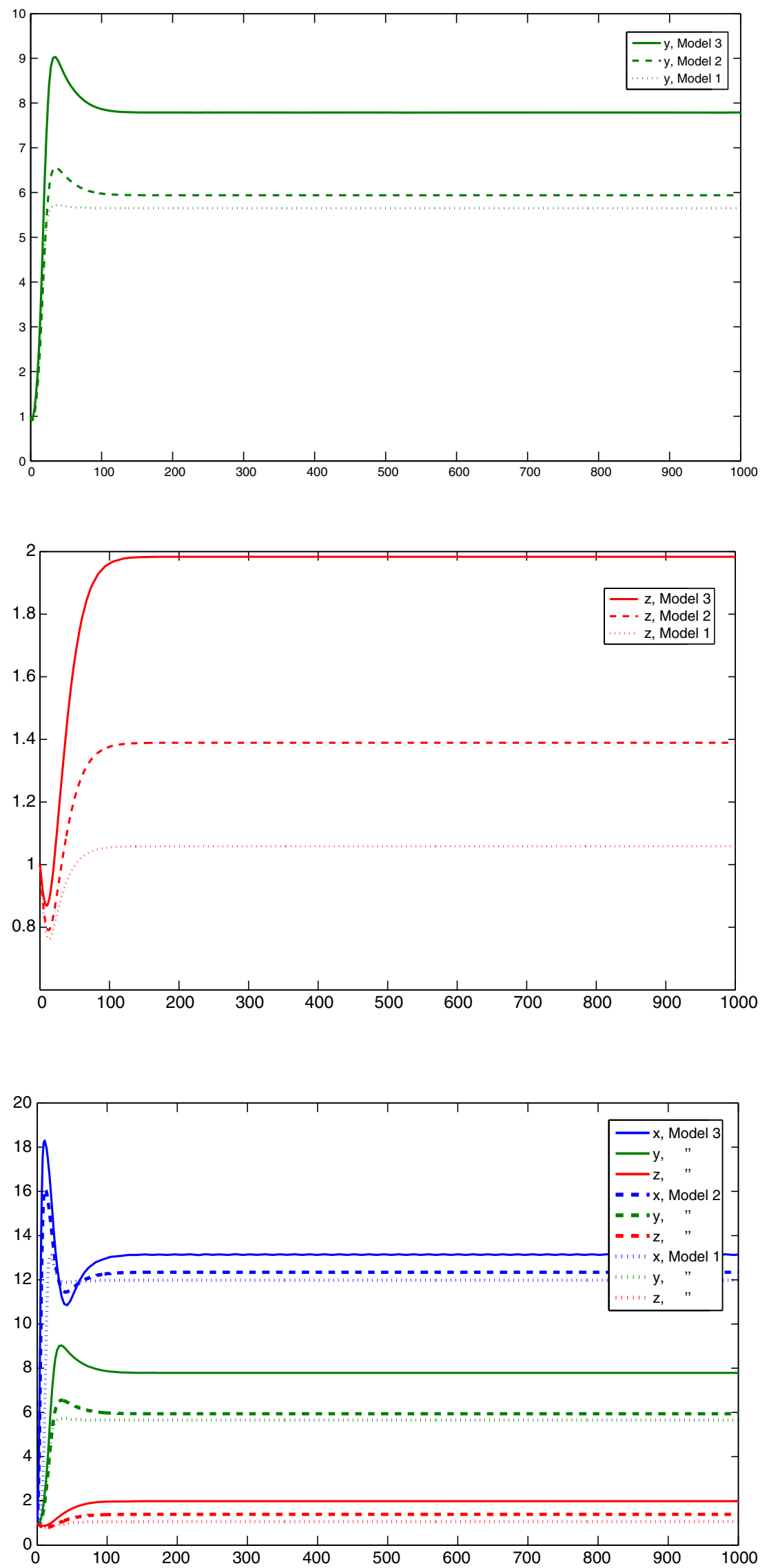


\section{Appendix A: Proof of Theorem 5}

Now, we will study the Hopf-bifurcation (Khare et al. 2011; Misra et al. 2013) of the system of Eqs. (7)-(11), taking $s_{1}$ as the bifurcation parameter. Now, the necessary and sufficient condition for the existence of the Hopf-bifurcation, if it exists is $s_{1}=s_{1}^{* *}$ such that

(i) $Q_{i}\left(s_{1}^{* *}\right)>0, i=1,2,3,4,5$,

(ii) $Q_{1}\left(s_{1}^{* *}\right) Q_{2}\left(s_{1}^{* *}\right)>Q_{3}\left(s_{1}^{* *}\right)$,

(iii) $Q_{1}\left(s_{1}^{* *}\right) Q_{2}\left(s_{1}^{* *}\right) Q_{3}\left(s_{1}^{* *}\right)>\left(Q_{3}\left(s_{1}^{* *}\right)^{2}+Q_{1}\left(s_{1}^{* *}\right)^{2}\right.$ $\left.Q_{4}\left(s_{1}^{* *}\right)\right)$,

(iv) $\quad\left(Q_{3}\left(s_{1}^{* *}\right) Q_{4}\left(s_{1}^{* *}\right) \quad-Q_{2}\left(s_{1}^{* *}\right) Q_{5}\left(s_{1}^{* *}\right)\right)\left(Q_{1}\left(s_{1}^{* *}\right) \quad Q_{2}\right.$ $\left.\left(s_{1}^{* *}\right)-Q_{3}\left(s_{1}^{* *}\right)\right)-\left(Q_{1}\left(s_{1}^{* *}\right) Q_{4}\left(s_{1}^{* *}\right)-Q_{5}\left(s_{1}^{* *}\right)\right)^{2}=$ 0 and

(v) if we consider the eigen values of the characteristic Eq. (90) is of the form $\lambda_{i}=u_{i}+i v_{i}$, then $\frac{d u_{i}}{d s_{1}} \neq 0, \quad i=1,2,3,4,5$. After substituting the values, the condition $\left(Q_{1} Q_{2}-Q_{3}\right)\left(Q_{3} Q_{4}-Q_{2}\right.$ $\left.Q_{5}\right)-\left(Q_{1} Q_{4}-Q_{5}\right)^{2}$ becomes

$$
D_{11} s_{1}^{3}+D_{12} s_{1}^{2}+D_{13} s_{1}+D_{14}=0
$$

where,

$$
\begin{aligned}
D_{11}= & \left(R_{14} R_{16}-R_{12} R_{18}\right)\left(Q_{1} R_{12}+R_{14}\right), \\
D_{12}= & Q_{1} R_{12}\left(R_{13} R_{16}-R_{14} R_{15}\right)+R_{14}\left(R_{11} R_{18}-R_{14} R_{15}\right) \\
& +2 R_{16}\left(R_{13} R_{14}-Q_{1} R_{18}\right) \\
& +Q_{1} R_{11}\left(2 R_{12} R_{18}-R_{14} R_{16}\right)-Q_{1}\left(Q_{1} R_{16}^{2}+R_{12}^{2} R_{17}\right) \\
& -R_{12}\left(R_{13} R_{18}+R_{14} R_{17}\right)-R_{18}^{2}, \\
D_{13}= & 2 Q_{1} R_{17}\left(R_{11} R_{12}-R_{16}\right)+\left(Q_{1} R_{15}+R_{17}\right)\left(R_{11} R_{14}-R_{12} R_{13}\right) \\
& +2 R_{18}\left(Q_{1} R_{15}-R_{17}\right)+2 R_{15}\left(Q_{1}^{2} R_{16}-R_{13} R_{14}\right) \\
& -R_{11} R_{13}\left(Q_{1} R_{16}-R_{18}\right)+R_{13}^{2} R_{16}-Q_{1} R_{11}^{2} R_{18}, \\
D_{14}= & \left(Q_{1} R_{11}-R_{13}\right)\left(R_{13} R_{15}-R_{11} R_{17}\right) \\
& +Q_{1} R_{15}\left(2 R_{17}-Q_{1} R_{15}\right)-R_{17}^{2}, \\
&
\end{aligned}
$$

$R_{11}=q_{12} q_{13}+x^{* *} q_{11} y^{* *} q_{14}+q_{15} q_{16}-\left(x^{* *} q_{11}+y^{* *} q_{14}\right)$ $d_{2} z^{* *}-\left(x^{* *} q_{11}+y^{* *} q_{14}-d_{2} z^{* *}\right) q_{17}-v a_{2}\left(x^{* *}\right)^{2}+\left(d_{2} z^{* *}\right.$ $\left.+q_{17}-x^{* *} q_{11}-y^{* *} q_{14}\right) q_{18}$,

$R_{12}=\quad v x^{* *} C_{0}^{* *}, \quad R_{13}=\left(q_{12} q_{13}+x^{* *} q_{11} y^{* *} q_{14}\right) d_{2} z^{* *}$ $-x^{* *} q_{11} q_{15} q_{16}+\left(q_{12} q_{13}+x^{* *} q_{11} y^{* *} q_{14}+q_{15} q_{16}-x^{* *} q_{11}\right.$ $\left.d_{2} z^{* *}-y^{* *} q_{14} d_{2} z^{* *}\right) q_{17}+\left(q_{12} q_{13}+x^{* *} q_{11} y^{* *} q_{14}+q_{15} q_{16}-\right.$ $x^{* *} q_{11} d_{2} z^{* *}-y^{* *} q_{14} d_{2} z^{* *}-x^{* *} q_{11} q_{17}-y^{* *} q_{14} q_{17}+d_{2} z^{* *}$ $\left.q_{17}\right) q_{18}+\left(q_{11} v\left(x^{* *}\right)^{2}+y^{* *} q_{14} v x^{* *}-d_{2} z^{* *} v x^{* *}\right) a_{2} x^{* *}$,

$$
R_{14}=a_{2} x^{* * 2}\left(\nu C_{0}^{* *}-a_{2} C_{E}^{* *}\right)+\left(y^{* *} q_{14}-d_{2} z^{* *}-q_{17}\right) x^{* *}
$$
$v C_{0}^{* *}$,

$R_{15}=q_{12} q_{13} d_{2} z^{* *} q_{17}+x^{* *} q_{11} y^{* *} q_{14} d_{2} z^{* *} q_{17}-x^{* *} q_{11}$ $q_{15} q_{16} q_{17}+\left(x^{* *} q_{11} d_{2} z^{* *}+y^{* *} q_{14} d_{2} z^{* *}-q_{12} q_{13}-x^{* *} q_{11}\right.$ $\left.y^{* *} q_{14}-q_{15} q_{16}\right) v a_{2} x^{* * 2}-x^{* *} q_{11} q_{15} q_{16} q_{18}+\left(q_{12} q_{13}+x^{* *}\right.$ $\left.q_{11} y^{* *} q_{14}\right) d_{2} z^{* *} q_{18}+\left(q_{12} q_{13}+x^{* *} q_{11} y^{* *} q_{14}+q_{15} q_{16}-x^{* *}\right.$ $\left.q_{11} d_{2} z^{* *}-y^{* *} q_{14} d_{2} z^{* *}\right) q_{17} q_{18}$,

$R_{16}=\left(d_{2} z^{* *}-y^{* *} q_{14}\right) x^{* *} q_{17} v C_{0}^{* *}-\left(y^{* *} q_{14} d_{2} z^{* *}-q_{15}\right.$ $\left.q_{16}\right) x^{* *} v C_{0}^{* *}-\left(d_{2} z^{* *}-y^{* *} q_{14}\right)\left(v C_{0}^{* *}-a_{2} C_{E}^{* *}\right) a_{2}\left(x^{* *}\right)^{2}$,

$R_{17}=\left(x^{* *} q_{11} q_{15} q_{16}-q_{12} q_{13} d_{2} z^{* *}-x^{* *} q_{11} y^{* *} q_{14} d_{2} z^{* *}\right)$ $v x^{* *} \quad a_{2} x^{* *}-x^{* *} q_{11} q_{15} q_{16} q_{17} q_{18}+\left(q_{12} q_{13}+x^{* *} q_{11} y^{* *} q_{14}\right)$ $d_{2} z^{* *} q_{17} q_{18}$,

$R_{18}=x^{* *}\left(y^{* *} q_{14} d_{2} z^{* *}-q_{15} q_{16}\right)\left(a_{1} v C_{0}^{* *}+a_{2}^{2} C_{E}^{* *} x^{* *}\right)$.

The Eq. (118) has at least one positive root say $s_{1}=s_{1}^{* *}$. Therefore, one pair of eigenvalues of the characteristic Eq. (90) at $s_{1}=s_{1}^{* *}$ are of the form $\lambda_{1,2}= \pm i v$, where $v$ is positive real number. Now, we will verify the Hopf-bifurcation condition ( $v$ ), putting $\lambda=u+i v$ in (90) and separating real and imaginary parts, we have

$$
\begin{aligned}
u^{5} & +Q_{1} u^{4}+\left(Q_{2}-10 v^{2}\right) u^{3}+\left(Q_{3}-6 Q_{1} v^{2}\right) u^{2} \\
& +\left(5 v^{4}-3 Q_{2} v^{2}+Q_{4}\right) u+\left(Q_{1} v^{4}-Q_{3} v^{2}+Q_{5}\right)=0
\end{aligned}
$$

$$
\begin{aligned}
& \left(v^{2}\right)^{2}-\left(10 u^{2}+4 Q_{1} u+Q_{2}\right) v^{2}+\left(5 u^{4}+4 Q_{1} u^{3}\right. \\
& \left.\quad+3 Q_{2} u^{2}+2 Q_{3} u+Q_{4}\right)=0
\end{aligned}
$$

Substituting the value of $v^{2}$ from (120) in (119), we get

$$
\begin{gathered}
u^{5}+Q_{1} u^{4}+\left(Q_{2}-10 p(u)\right) u^{3}+\left(Q_{3}-6 Q_{1} p(u)\right) u^{2} \\
+\left(5(p(u))^{2}-3 Q_{2} p(u)+Q_{4}\right) u+\left(Q_{1}(p(u))^{2}\right. \\
\left.\quad-Q_{3} p(u)+Q_{5}\right)=0
\end{gathered}
$$

where $p(u)=\frac{1}{2}\left(\left(10 u^{2}+4 Q_{1} u+Q_{2}\right)-G_{20}\right)$ and $G_{20}=$ $\sqrt{\left(10 u^{2}+4 Q_{1} u+Q_{2}\right)^{2}-4\left(5 u^{4}+4 Q_{1} u^{3}+3 Q_{2} u^{2}+2 Q_{3} u+Q_{4}\right)}$, differentiating with respect to $s_{1}$ and putting $s_{1}=s_{1}^{* *}$, we have

$$
\begin{aligned}
& {\left[\frac{d u}{d s_{1}}\right]_{s_{1}=s_{1}^{* *}}} \\
& \quad=\frac{p(0) \frac{d Q_{3}}{d s_{1}}-(p(0))^{2} \frac{d Q_{1}}{d s_{1}}-\frac{d Q_{5}}{d s_{1}}}{5(p(0))^{2}+2 Q_{1} p(0) p^{\prime}(0)-3 Q_{2} p(0)-Q_{3} p^{\prime}(0)+Q_{4}} \neq 0
\end{aligned}
$$

since,

$$
\begin{aligned}
& p\left(s_{1}\right) \frac{d Q_{3}}{d s_{1}}-\left(p\left(s_{1}\right)\right)^{2} \frac{d Q_{1}}{d s_{1}}-\frac{d Q_{5}}{d s_{1}}=p(u)\left[v x ^ { * * } C _ { 0 } ^ { * * } \left(a_{2} x^{* *}\right.\right. \\
& \left.\left.\quad+y^{* *} q_{14}-z^{* *} d_{1}-q_{17}\right)-a_{2}^{2} x^{* * 2} C_{E}^{* *}\right] \\
& \quad-\left[x^{* *}\left(y^{* *} z^{* *} d_{2} q_{14}-q_{15} q_{16}\right)\left(v a_{1} C_{0}^{* *}+x^{* *} C_{E}^{* *} a_{2}^{2}\right)\right] \neq 0
\end{aligned}
$$

For example, taking $s_{0}=0.7845, k=25, q_{1}=0.47, q_{2}=$ $0.334, q_{3}=0.381, q_{4}=0.12, r_{0}=0.02423, r_{1}=0.0257$, $s_{1}=0.06, d_{1}=0.0571, \quad d_{2}=0.02483, q_{0}=0.515, a_{1}=$ $0.71, a_{2}=0.92, \quad v=0.102$, and $b_{1}=0.52$, in the Eq. (122), we get a positive value i.e., 0.313506 . 
This ensures that the above system has a Hopf-bifurcation around the positive interior equilibrium $E_{23}^{* *}$.

\section{Appendix B: Proof of Theorem 6}

We consider the following positive definite function about $E_{23}^{* *}$ :

$$
\begin{aligned}
V_{24}= & \left(x-x^{* *}-x^{* *} \ln \left(\frac{x}{x^{* *}}\right)\right)+H_{1}\left(y-y^{* *}-y^{* *} \ln \left(\frac{y}{y^{* *}}\right)\right) \\
& +\left(z-z^{* *}-z^{* *} \ln \left(\frac{z}{z^{* *}}\right)\right)+\frac{H_{2}}{2}\left(C_{E}-C_{E}^{* *}\right)^{2} \\
& +\frac{H_{3}}{2}\left(C_{0}-C_{0}^{* *}\right)^{2} \\
\frac{d V_{24}}{d t}= & \left(\frac{x-x^{* *}}{x}\right) \frac{d x}{d t}+H_{1}\left(\frac{y-y^{* *}}{y}\right) \frac{d y}{d t}+\left(\frac{z-z^{* *}}{z}\right) \frac{d z}{d t} \\
& +H_{2}\left(C_{E}-C_{E}^{* *}\right) \frac{d C_{E}}{d t}+H_{3}\left(C_{0}-C_{0}^{* *}\right) \frac{d C_{0}}{d t} \\
\frac{d V_{24}}{d t}= & -\left(x-x^{* *}\right)^{2}\left(\frac{1}{k}-\frac{q_{1} y^{*}}{\pi_{21}}\right)-\left(y-y^{* *}\right)^{2} \\
& H_{1}\left(r_{1}+\frac{q_{3} z^{* *}}{\pi_{22}}\right)-\left(z-z^{* *}\right)^{2} d_{2} \\
- & \left(C_{E}-C_{E}^{* *}\right)^{2} H_{2}\left[a_{1}+\left(a_{2}-v\right) x^{* *}\right] \\
& -\left(C_{0}-C_{0}^{* *}\right)^{2} H_{3}\left(b_{1}+v x^{* *}\right) \\
- & \left(x-x^{* *}\right)\left(y-y^{* *}\right)\left[\left(1+x^{* *}\right) q_{1}-H_{1} q_{2}\right] / \pi_{21} \\
- & \left(x-x^{* *}\right)\left(C_{E}-C_{E}^{* *}\right) H_{2}\left[a_{2} C_{E}-v C_{0}\right] \\
& +\left(C_{E}-C_{E}^{* *}\right)\left(C_{0}-C_{0}^{* *}\right) H_{3} a_{2} x^{* *} \\
+ & \left(x-x^{* *}\right)\left(C_{0}-C_{0}^{* *}\right)\left[H_{3}\left(a_{2} C_{E}-v C_{0}\right)-s_{1}\right] \\
+ & \left(y-y^{* *}\right)\left(z-z^{* *}\right)\left[\left(1+y^{* *}\right) H_{1} q_{3}+H_{2} q_{4}\right] / \pi_{22}
\end{aligned}
$$

Now using the sylvester's criterion in the quadratic forms

$$
\begin{aligned}
\frac{d V_{24}}{d t} \leq- & {\left[\left(\left(h_{11} / 3\right)\left(x-x^{* *}\right)^{2}+h_{12}\left(x-x^{* *}\right)\left(y-y^{* *}\right)\right.\right.} \\
& \left.+\left(h_{22} / 2\right)\left(y-y^{* *}\right)^{2}\right) \\
+ & \left(\left(h_{11} / 3\right)\left(x-x^{* *}\right)^{2}+h_{14}\left(x-x^{* *}\right)\left(C_{E}-C_{E}^{* *}\right)\right. \\
& \left.+\left(h_{44} / 2\right)\left(C_{E}-C_{E}^{* *}\right)^{2}\right) \\
+ & \left(\left(h_{11} / 3\right)\left(x-x^{* *}\right)^{2}-h_{15}\left(x-x^{* *}\right)\left(C_{0}-C_{0}^{* *}\right)\right. \\
& \left.+\left(h_{55} / 2\right)\left(C_{0}-C_{0}^{* *}\right)^{2}\right) \\
+ & \left(\left(h_{22} / 2\right)\left(y-y^{* *}\right)^{2}-h_{23}\left(y-y^{* *}\right)\left(z-z^{* *}\right)\right. \\
& \left.+h_{33}\left(z-z^{* *}\right)^{2}\right) \\
+ & \left(\left(h_{44} / 2\right)\left(C_{E}-C_{E}^{* *}\right)^{2}-h_{45}\left(C_{E}-C_{E}^{* *}\right)\right. \\
& \left.\left.\left(C_{0}-C_{0}^{* *}\right)+\left(h_{55} / 2\right)\left(C_{0}-C_{0}^{* *}\right)^{2}\right)\right]
\end{aligned}
$$

where,

$$
\begin{aligned}
h_{11}= & \left(\frac{1}{k}-\frac{q_{1} y^{*}}{\pi_{21}}\right) / 3, h_{12}=\left[\left(1+x^{* *}\right) q_{1}-H_{1} q_{2}\right] / \pi_{21}, \\
& h_{14}=H_{2}\left[a_{2} C_{E}-v C_{0}\right], \\
h_{15}= & H_{3}\left(a_{2} C_{E}-v C_{0}\right)-s_{1}, h_{22}=H_{1}\left(r_{1}+\frac{q_{3} z^{* *}}{\pi_{22}}\right) / 2, \\
& \pi_{22}=(1+y)\left(1+y^{* *}\right) \\
h_{23}= & {\left[\left(1+y^{* *}\right) H_{1} q_{3}+H_{2} q_{4}\right] / \pi_{22}, h_{33}=d_{2}, } \\
& h_{44}=H_{2}\left[a_{1}+\left(a_{2}-v\right) x^{* *}\right] / 2, \\
h_{45}= & H_{3} a_{2} x^{* *}, h_{55}=H_{3}\left(b_{1}+v x^{* *}\right) / 2, \\
& \pi_{21}=(1+x)\left(1+x^{* *}\right) .
\end{aligned}
$$

Sufficient conditions for $d V_{34} / d t$ to be negative definite are that the following inequalities hold:

$$
\begin{aligned}
& h_{11}>0 \\
& h_{44}>0 \\
& h_{11} h_{22}>h_{12}^{2} \\
& h_{11} h_{44}>h_{14}^{2} \\
& h_{11} h_{55}>h_{15}^{2} \\
& h_{22} h_{33}>h_{23}^{2} \\
& h_{44} h_{55}>h_{45}^{2}
\end{aligned}
$$

We note that the fifth, sixth and eighth inequalities, i.e., $h_{11} h_{22}>h_{12}^{2}, h_{11} h_{44}>h_{14}^{2}$ and $h_{11} h_{55}>h_{15}^{2}$ are satisfied due to the proper choice of $H_{1}, H_{2}$ and $H_{3}$ and other inequalities, (91) $\Rightarrow$ (123), (92) $\Rightarrow(124),(93) \Rightarrow(128)$ and $(94) \Rightarrow(129)$. Hence $V_{24}$ is a Liapunov function with respect to $E_{23}^{* *}$, whose domain contains the region of attraction $\Omega$, proving the theorem.

\section{Appendix C: Proof of Theorem 7}

Now, we will study the Hopf-bifurcation (Khare et al. 2011; Misra et al. 2013) of the system of Eqs. (12)-(14) taking $q_{4}$ as the bifurcation parameter. The necessary and sufficient conditions for the existence of the Hopf-bifurcation for $q_{4}=\tilde{q_{4}}$, if it exist are

(i) $B_{i}\left(\tilde{q_{4}}\right)>0, i=1,2,3$

(ii) $\quad B_{1}\left(\tilde{q_{4}}\right) B_{2}\left(\tilde{q_{4}}\right)-B_{3}\left(\tilde{q_{4}}\right)=0$ and

(iii) the eigenvalues of the characteristic equation should be of the form $\lambda_{i}=u_{i}+i v_{i}$, and $\frac{d u_{i}}{d q_{4}} \neq 0$, 
$i=1,2,3$. After substituting the values, the condition $G_{10}=B_{1} B_{2}-B_{3}$ becomes

$$
-D_{1} q_{4}^{3}+D_{2} q_{4}^{2}+D_{3} q_{4}+D_{4}=0
$$

where, $D_{1}=V_{1} V_{3}, D_{2}=V_{6}+V_{1} V_{4}-V_{2} V_{3}, D_{3}$ $=V_{2} V_{4}+V_{1} V_{5}-V_{7}, D_{4}=V_{2} V_{5}+V_{8}, \quad V_{1}=\frac{\tilde{y}}{1+\tilde{y}}$ $\left(1-\frac{q_{3} \tilde{y}}{d_{2}(1+\tilde{y})^{2}}\right), \quad V_{2}=\tilde{x}\left(\frac{1}{k}-\frac{q_{1} \tilde{y}}{(1+\tilde{x})^{2}}\right) \quad+r_{1} \tilde{y}-d_{1}+$ $\frac{d_{1} q_{3} \tilde{y}}{d_{2}(1+\tilde{y})^{2}}, \quad V_{3}=(\tilde{y}-1) \frac{q_{3} \tilde{y}^{2}}{d_{2}(1+\tilde{y})^{4}}, \quad V_{4}=\frac{\tilde{y}^{2}}{1+\tilde{y}} \quad\left(r_{1}+\right.$ $\left.\frac{d_{1} q_{3}}{d_{2}(1+\tilde{y})^{2}}\right)-\frac{d_{1} q_{3} \tilde{y}}{d_{2}(1+\tilde{y})^{3}}+\frac{\tilde{x} \tilde{y}}{1+\tilde{y}}\left(1-\frac{q_{3} \tilde{y}}{d_{2}(1+\tilde{y})^{2}}\right)\left(\frac{1}{k}-\frac{q_{1} \tilde{y}}{(1+\tilde{x})^{2}}\right)$, $V_{5}=\tilde{x}\left(r_{1} \tilde{y}-d_{1}\right)\left(\frac{1}{k}-\frac{q_{1} \tilde{y}}{(1+\tilde{x})^{2}}\right)+\frac{q_{1} q_{2} \tilde{x} \tilde{y}}{(1+\tilde{x})^{3}}+\frac{d_{1} q_{3} \tilde{y}}{d_{2}(1+\tilde{y})^{2}}, V_{6}$ $=\frac{(\tilde{y}-1) q_{3} \tilde{y} \tilde{y}^{2}}{d_{2}(1+\tilde{y})^{4}}\left(\frac{1}{k}-\frac{q_{1} \tilde{y}}{(1+\tilde{x})^{2}}\right), \quad V_{7}=\frac{q_{1} q_{2} \tilde{x} \tilde{y}^{2}}{(1+\tilde{x})^{3}(1+\tilde{y})}+\frac{q_{1} \tilde{y} \tilde{y}^{2}}{d_{2}(1+\tilde{y})}$ $\left(r_{1} d_{2}+\frac{d_{1} q_{3}}{(1+\tilde{y})^{2}}\right) \quad\left(\frac{1}{k}-\frac{q_{1} \tilde{y}}{(1+\tilde{x})^{2}}\right)+(\tilde{y}-1) \frac{\tilde{y} \tilde{y} d_{1} q_{3}}{(1+\tilde{y})^{3}} \quad\left(\frac{1}{k}-\right.$ $\left.\frac{q_{1} \tilde{y}}{(1+\tilde{x})^{2}}\right), \quad V_{8}=\frac{d_{1} q_{1} q_{2} \tilde{x} \tilde{y}}{(1+\tilde{x})^{3}}+\quad \frac{d_{1} \tilde{x} \tilde{y}}{d_{2}}\left(r_{1} d_{2}+\frac{d_{1} q_{3}}{(1+\tilde{y})^{2}}\right) \quad\left(\frac{1}{k}-\right.$ $\left.\frac{q_{1} \tilde{y}}{(1+\tilde{x})^{2}}\right)$. The Eq. (130) has at least one positive root say $q_{4}=\tilde{q_{4}}$. Therefore, one pair of eigenvalues of the characteristic Eq. (115) at $q_{4}=\tilde{q_{4}}$ are of the form $\lambda_{1,2}= \pm i v$, where $v$ is positive real number.

Now, we will verify the Hopf-bifurcation condition (iii), putting $\lambda=u+i v$ in (115), we get

$$
(u+i v)^{3}+B_{1}(u+i v)^{2}+B_{2}(u+i v)+B_{3}=0
$$

On separating the real and imaginary parts, we have

$u^{3}-3 u v^{2}+B_{1} u^{2}-B_{1} v^{2}+B_{2} u+B_{3}=0$

$-v^{2}+3 u^{2}+2 B_{1} u+B_{2}=0$

Substituting the value of $v^{2}$ from (133) in (132), we get

$$
8 u^{3}+8 B_{1} u^{2}+2\left(B_{1}^{2}+B_{2}\right) u+\left(B_{1} B_{2}-B_{3}\right)=0
$$

It is clear from the above that $u\left(\tilde{q_{4}}\right)=0$ iff $B_{1}\left(\tilde{q_{4}}\right) B_{2}\left(\tilde{q_{4}}\right)-B_{3}\left(\tilde{q_{4}}\right)=0$. Further, at $q_{4}=\tilde{q_{4}}, u\left(\tilde{q_{4}}\right)$ is the only root, since the discriminate of $8 u^{2}+8 B_{1} u+$ $2\left(B_{1}^{2}+B_{2}\right)=0$ is $64 B_{1}^{2}-64\left(B_{1}^{2}+B_{2}\right)<0$. Again, differentiating (134) with respect to $q_{4}$, we have

$$
\begin{aligned}
24 u^{2} \frac{d u}{d q_{4}} & +16 B_{1} u \frac{d u}{d q_{4}}+8 u^{2} \frac{d B_{1}}{d q_{4}}+2\left(B_{1}^{2}+B_{2}\right) \frac{d u}{d q_{4}} \\
& +2 u\left[2 B_{1} \frac{d B_{1}}{d q_{4}}+\frac{d B_{2}}{d q_{4}}\right]+\frac{d G_{10}}{d q_{4}}=0
\end{aligned}
$$

Now, since at $q_{4}=\tilde{q_{4}}, u\left(\tilde{q_{4}}\right)=0$ we get

$$
\left[\frac{d u}{d q_{4}}\right]_{q_{4}=\tilde{q_{4}}}=\frac{-\frac{d G_{10}}{d q_{4}}}{2\left(B_{1}^{2}+B_{2}\right)} \neq 0
$$

This ensures that the above system has a Hopf-bifurcation around the positive interior equilibrium $\tilde{E_{33}}$.

\section{Appendix D: Proof of Theorem 8}

We consider the following positive definite function about $\tilde{E_{33}}$ :

$$
\begin{aligned}
V_{14} & =\left(x-\tilde{x}-\tilde{x} \ln \left(\frac{x}{\tilde{x}}\right)\right)+I_{1}\left(y-\tilde{y}-\tilde{y} \ln \left(\frac{y}{\tilde{y}}\right)\right) \\
+ & I_{2}\left(z-\tilde{z}-\tilde{z} \ln \left(\frac{z}{\tilde{z}}\right)\right)
\end{aligned}
$$

Differentiating $V_{14}$ with respect to time $t$, we get

$$
\begin{aligned}
\frac{d V_{14}}{d t} & =\left(\frac{x-\tilde{x}}{x}\right) \frac{d x}{d t}+I_{1}\left(\frac{y-\tilde{y}}{y}\right) \frac{d y}{d t}+I_{2}\left(\frac{z-\tilde{z}}{z}\right) \frac{d z}{d t} \\
\frac{d V_{14}}{d t} & =-(x-\tilde{x})^{2}\left(\frac{1}{k}-\frac{q_{1} \tilde{y}}{\sigma_{1}}\right)-(y-\tilde{y})^{2} I_{1}\left(r_{1}-\frac{\tilde{z} q_{3}}{\sigma_{2}}\right)-I_{2}(z-\tilde{z})^{2} d_{2} \\
& +(x-\tilde{x})(y-\tilde{y}) \frac{1}{\sigma_{1}}\left(q_{2} I_{1}-q_{1}(1+\tilde{x})\right) \\
& +(y-\tilde{y})(z-\tilde{z}) \frac{1}{\sigma_{2}}\left(q_{4} I_{2}-I_{1} q_{3}(1+\tilde{y})\right) .
\end{aligned}
$$

Assuming $I_{1}=\frac{q_{1}}{q_{2}}(1+\tilde{x}) \quad$ and $\quad I_{2}=\frac{q_{1} q_{3}}{q_{2} q_{4}}(1+\tilde{x})(1+\tilde{y})$, where $\sigma_{1}=(1+x)(1+\tilde{x}), \sigma_{2}=(1+y)(1+\tilde{y})$.

$$
\begin{aligned}
\frac{d V_{14}}{d t}= & -(x-\tilde{x})^{2}\left(\frac{1}{k}-\frac{q_{1} \tilde{y}}{\sigma_{1}}\right)-(y-\tilde{y})^{2} I_{1}\left(r_{1}-\frac{\tilde{z} q_{3}}{\sigma_{2}}\right) \\
& -I_{2}(z-\tilde{z})^{2} d_{2}
\end{aligned}
$$

which is negative definite if in $\Omega$ requiring that: $\frac{1}{k}-$ $\frac{q_{1} \tilde{y}}{1+\tilde{x}}>0$ and $r_{1}-\frac{\tilde{z} q_{3}}{1+\tilde{y}}>0$. Hence $V_{14}$ is a Liapunov function with respect to $\tilde{E_{33}}$, whose domain contains the region of attraction $\Omega$, proving the theorem.

\section{References}

Arino O, Hbid ML, Dads EA (eds) (2007) Delay differential equations and applications. Proceedings of the NATO advanced study institute held in Marrakech, Morocco, 9-21 Sept 2002, vol 205. Springer

Bernard S, Belair J, Mackey MC (2001) Sufficient conditions for stability of linear differential equations with distributed delay. Discrete Continuous Dyn Syst Ser B 2:233-256

Dubey B, Hussain J (2006) Modelling the survival of species dependent on a resource in a polluted environment. Nonlinear Anal Real World Appl 7:187-210

Gakkhar S, Sahani SK (2009) A model for delayed effect of toxicant on resource-biomass system. Chaos Solitons Fractals 40:912-922

Hallam TG, De Luna JT (1984) Effects of toxicants on populations: a qualitative approach III. Environmental and food chain pathways. J Theor Biol 109(3):411-429

He X-Z, Ruan S (1998) Global stability in chemostat-type plankton models with delayed nutrient recycling. J Math Biol 37:253-271

Hladun KR, Smith BH, Mustard JA, Morton RR, Trumble JT (2012) Selenium toxicity to honey bee (Apis mellifera L.) pollinators: effects on behaviors and survival. Plos One 7(4):e34137 
Khare S, Misra OP, Dhar J (2009) Population dynamics modelling with nutrient cycling and pollutants. LAP LAMBERT Academic Publishing. ISBN: 978-3-659-30873-4

Khare S, Misra OP, Singh C, Dhar J (2011) Role of delay on planktonic ecosystem in the presence of a toxic producing phytoplankton. Int J Differ Equ, Article ID 603183, 16 pages. doi:10.1155/2011/603183

Liu WM (1994) Criterion of Hopf-bifurcations without using eigenvalues. J Math Anal Appl 182:250-256

Ma W, Takeuchi Y (1998) Stability analysis on a predator-prey system with distributed delays. J Comput Appl Math 88:79-94

Misra OP, Sinha P, Singh C (2013) Stability and bifurcation analysis of a preypredator model with age based predation. Appl Math Model 37:6519-6529

Perez-Legaspi IA, Rico-Martnez R (2001) Acute toxicity tests on three species of the genus Lecane (Rotifera: Monogononta). Hydrobiologia 446(447):375-381

Rasmusse H, Wake GC, Donaldson J (2003) Analysis of a class of distributed delay logistic differential equations. Math Comput Model 38:123-132

Ruan S, Wolkowicz SK (1996) Bifurcation analysis of a chemostat model with a distributed delay. J Math Anal Appl 204:786-812

Shi R, Qi J, Tang S (2013) Stability and bifurcation analysis for a predator-prey model with discrete and distributed delay. In:
Abstract and applied analysis, Article ID 454097. Hindawi publishing corporation

Shukla JB, Dubey B (1997) Modelling the depletion and conseration of forestry resources: effects of population and pollution. J Math Biol 36:71-94

Tabares PCC, Ferreira JD, Sree Hari Rao V (2011) Weak Allee effect in a predator-prey system involving distributed delays. Comput Appl Math 3:675-699

Thomann RV, Connolly JP (1984) Model of PCB in the lake michigan lake trout these food chain. Environ Sci Technol 18:65-71

Thomann RV, Szumski DS, Ditoto DM, O'Connor DJ (1984) A food chain model of cadmium in western lake Erie. Water Res 8:841-849

Turner JT, Tester PA (1997) Toxic marine phytoplankton, zooplankton grazers, and pelagic food webs. Liminol Oceanogr 42:1203-1214

Wang F, Pang G (2008) Chaos and Hopf bifurcation of a hybrid ratiodependent three species food chain. Chaos Solitions Fractals 36:1366-1376

Zhang P, Sun J, Chen J, Wei J, Zhao W, Liu Q, Sun H (2013) Effect of feeding selectivity on the transfer of methylmercury through experimental marine food chains. Mar Environ Res 89:39-44 